\title{
PLETHORA OF PLANTS - COLLECTIONS OF THE BOTANICAL GARDEN, FACULTY OF SCIENCE, UNIVERSITY OF ZAGREB (1): TEMPERATE GLASSHOUSE EXOTICS - HISTORIC OVERVIEW
}

\begin{abstract}
Sanja Kovačić
Botanical Garden, Department of Biology, Faculty of Science, University of Zagreb, Marulićev trg 9a, HR-10000 Zagreb, Croatia (e-mail: sanja.kovacic@biol.pmf.hr)
\end{abstract}

Kovačić, S.: Plethora of plants - collections of the Botanical garden, Faculty of Science, University of Zagreb (1): Temperate glasshouse exotics - historic overview. Nat. Croat., Vol. 24, No. 2, $361-397^{*}, 2015$, Zagreb

Due to the forthcoming obligation to thoroughly catalogue and officially register all living and non-living collections in the European Union, an inventory revision of the plant collections in Zagreb Botanical Garden of the Faculty of Science (University of Zagreb, Croatia) has been initiated. The plant lists of the temperate (warm) greenhouse collections since the construction of the first, Exhibition Glasshouse (1891), until today (2015) have been studied. Synonymy, nomenclature and origin of plant material have been sorted. Lists of species grown (or that presumably lived) in the warm greenhouse conditions during the last 120 years have been constructed to show that throughout that period at least 1000 plant taxa from 380 genera and 90 families inhabited the temperate collections of the Garden. Today, that collection holds 320 exotic taxa from 146 genera and 56 families.

Key words: Zagreb Botanical Garden, warm greenhouse conditions, historic plant collections, temperate glasshouse collection

Kovačić, S.: Obilje bilja - zbirke Botaničkoga vrta Prirodoslovno-matematičkog fakulteta Sveučilišta u Zagrebu (1): Uresnice toplog staklenika - povijesni pregled. Nat. Croat., Vol. 24, No. 2, $361-397^{*}, 2015$, Zagreb

Slijedom najavljenog temeljitog popisivanja i službene registracije svih živih i neživih zbirki u Europskoj uniji, u Botaničkom vrtu Prirodoslovno-matematičkog fakulteta Sveučilišta u Zagrebu pokrenuta je revizija sadržaja stakleničkih zbirki. Sastavljeni su popisi biljaka toplog staklenika od njegovog podizanja (1891.) do danas (2015.), uređena sinonimka i nomenklatura te istraženo podrijetlo biljnog materijala. Rezultati pokazuju kako je kroz zbirku toplog staklenika tijekom posljednjih 120 godina prošlo barem 1000 svojti iz 380 rodova i 90 porodica. Danas u toj zbirci živi 320 biljnih svojti iz 146 rodova i 56 porodica.

Ključne riječi: Botanički vrt u Zagrebu, povijesne zbirke biljaka, staklenički uvjeti, zbirka toplog staklenika

\section{INTRODUCTION}

Established in 1889 and statutorily protected in 1971 as a Monument of Park Architecture and an integral part of the Zagreb Green Horseshoe Monument of Culture (RegulA,

\footnotetext{
* Supplementary Material: Table 2 is available only in the Electronic Supplement of the online version of this article.
}

Dodatni materijal: Tablica 2 dostupna je samo u Dodacima elektroničke verzije ovoga članka. 
1997; Juretić et al., 2014), the Botanical Garden of the Faculty of Science, University of Zagreb (in further text "the Botanical Garden" or "the Garden") today is a member of the Botanic Gardens Conservation International (BGCI) and International Plant Exchange Network (IPEN). Though very small (4.45 ha) and placed in the very heart of the Croatian Capital, it has a 125-years-long tradition of university education and international seed exchange via Delectus (Index) Seminum-publications. Along with the easily accessible outdoor areas, the Garden also has several glasshouses. The largest, Exhibition Glasshouse (Figs. 1abcd), is one of the oldest in this part of Europe. It was built in 1891 on the model of the famous Viennese Palmenhaus in Schönbrunn, and by the same craftsmen, in the same well-regarded Viennese master-workshop. Though nowadays closed to the public due to the long deterioration, the glasshouses are still home to many plants from all continents and were during the century a home to many more, now vanished.

As a part of the announced need to register all, both living and non-living, collections in the European Union, and in view of the forthcoming total reconstruction of the old Garden Exhibition Glasshouse, in 2015 I made an inventory of all existing and onceexisting plants of the temperate (warm) greenhouse collection. The main idea was to gain a combined and full inventory, which would provide, for a first time in the Garden's history, a thorough insight to the "houseplants" grown in the Botanical Garden's temperate collections (Figs. 2ab). After we published data on the Croatian statutorily strictly protected species grown in the Garden (cp. SANDEv et al., 2013; Kovačić et al., 2014), it is our intention, to systematically examine and register in such a manner all of our Garden collections.

\section{The Greenhouses}

Since the building of the first greenhouses of Emperor Tiberius in the $1^{\text {st }}$ century AD, via the Far East glasshouses of $15^{\text {th }}$ century Korea, $17^{\text {th }}$ century Netherlands and early $19^{\text {th }}$ century Europe (Woods \& Swartz Warren, 1990; AnOnymous, 2007; PARis \& Janick, 2008; BAvCON et al., 2012 and the literature within; GRANT, 2013), the "indoor-space for plants" became a standard of any botanic garden. Heated buildings with adequate humidity and ventilation become necessary for any garden that wishes to preserve plant material for research and education, as well as to grow exotics to attract more visitors.

In the $20^{\text {th }}$ century, it became common to classify greenhouses, according to the minimal winter temperatures provided, together with the required humidity and lightning, into "cool" (cold, frigidaria), "warm" (temperate, lepidaria), and "hot" (tropical, caldaria). Accordingly, in our Botanical Garden the "cool" glasshouse maintains a winter temperature below $12{ }^{\circ} \mathrm{C}$ or $10^{\circ} \mathrm{C}$, so light heating is required. Depending on the plant collection overwintering in such an environment, the amount of humidity should be levelled between very low (for example, for the collection of succulents) and relatively high (for the collection of Mediterranean plants). In our Garden, the "cool" glasshouses are used for the overwintering of Croatian and other Mediterranean native (potted) plants, as well as palms (fam. Arecaceae) and succulents (families Cactaceae, Mesembryanthemaceae, Euphorbiaceae etc.). In the "hot" glasshouses, the collections of tropical (and some subtropical) plants do not just overwinter, but in most of cases live permanently. These premises require a minimum all-year round temperature of at least $20^{\circ} \mathrm{C}$ to $18^{\circ} \mathrm{C}$. The regular daily temperature is often higher than $30^{\circ} \mathrm{C}$, to mimic the conditions of tropical and rain forests. Along with the high temperatures, hot glasshouses must have a relative humidity above $80 \%$, as well as rain-watering and appropriate misting- and shadingsystems, so they are expensive to maintain. 
In-between the "cool" and "hot" greenhouse conditions, there is a range of various so called "warm" or temperate ambiences, required for a vast spectrum of plants which we often call "the houseplants". The most common conditions are those of a "living room": the lowest temperature required during the wintertime is between $15^{\circ} \mathrm{C}$ and 18 ${ }^{\circ} \mathrm{C}$, along with a medium humidity and considerable shading at mid-day. In the temperate ("warm") glasshouse conditions, the maintenance of high humidity is appropriate, but not essential, as it is in the "hot" glasshouses. More important is regular ventilation: stale air is inappropriate and favourable only for the growth of various pests. However, draft is also harmful to the plants, so airing must be carried out with caution.

From the building of the Exhibition Glasshouse (1891) in our Botanical Garden, the "houseplants" and other exotics from temperate parts of the world were grown in the presentation area - later, presumably, in the "supplementary" glasshouse as well, which was erected in 1899 (REgula, 1997). In 1985, that smaller greenhouse was enlarged to approx. $150 \mathrm{~m}^{2}$ and colloquially named "The Aroideae" (Fig. 3), for it was initially intended for the members of Araceae family (especially subfam. Aroideae) and their relatives. Thirty years on, mostly due to the chronic shortage of space, a large number of various plants live there (Fig. 4), while the members of Araceae family dwell mostly in the tropical glasshouse.

In the warm greenhouse-conditions of the Garden today a collection of exotic plants from temperate and subtropical parts of the world (Australia, South America, Asia) overwinters and permanently lives; among these plants many are economically important (edible, medicinal, ornamental) and there are also popular ornamentals (cultivars, hybrids, forms).

The aim of this paper is to reveal the data on the plant species that have been grown in the temperate collections of the Botanical Garden (Faculty of Science, University of Zagreb) since its foundation. For establishing a list of associated taxa, during 2015 I thoroughly examined the published works, the Garden database and my own records. Lists of plants living in the temperate glasshouse collection between 1895 and 2015 were thus constructed and compared. The comprehensive inventory of the recent collection is a foundation for the announced thorough cataloguing and registration of all collections in the European Union. However, it is also important to discover the plants grown in the collections over the years, for by knowing both, we can plan our further steps in maintaining and supplementing the collections: replacing the missing parts of the puzzle, and adding new ones, to form a meaningful whole.

\section{MATERIAL \& METHOD}

Three main sources are used for constructing the lists of exotic plants growing in the Botanical Garden's temperate glasshouse collections, since its establishment: (i) published data on the historic collection of the early days (1891-1896), (ii) the passive database of plants cultivated in the Garden since 1948 but not living today, and (iii) active database of plants recently (2015) living in the temperate glasshouse collection, together with the attending gardener's glasshouse inventories.

The initial part of this study is based on a booklet written by the founder of the Botanical Garden, Professor Antun Heinz (1861-1919). In this work, published in 1895-1896, he depicts the astonishing amount of indoor- and outdoor-plants contained in the collections soon after the Garden was formally established (1889). It is quite hard to belie- 
ve that in only four years after "the first shovel was stuck into the bare ground" in early March of 1891 (HeInz, 1895-1896, pg. 13), such a plethora of plants inhabited the Garden facilities.

The booklet (HeInz, 1895-1896) was written according to the then valid classification of the Plant Kingdom, which is today somewhat hard to follow, and was quite vague with respect to the details in the inventory. In numerous cases, only plant genera are named, as "containing several/many species", without any further details (origin, acquirement, living conditions, etc.). Though back then the Garden Exhibition Glasshouse was divided in several temperature zones ("kuće") and the collections accordingly, Heinz mostly named the location in the Garden where the certain plant group lives just as "in the greenhouses" or "in the open", without information where exactly or in what conditions. Accordingly, Tab. 1 lists the plants grown in the temperate glasshouse collections in 1895. Since the precise data of Heinz (1895-1896) are missing, it must be stressed that much speculation went into the construction of Tab. 1: I had to make many "educated guesses" either as to the plant species that actually need the warm-glasshouse conditions to thrive, or the valid names of taxa of all ranks, which changed during the century. At first, I was guided by the data on life-requirements for the species listed by Heinz, or their relatives living conditions in our glasshouse collections today. Secondly, I kept the original names stated by Heinz, mainly for historical reasons, but with some notes on the currently valid nomenclature and classification, when possible. Though incomplete, this oldest inventory of the temperate greenhouse-plant collection ( $\mathrm{He}-$ INZ, 1895-1896) is worth mentioning in order to project a picture of the collection as initially established, and identify the plant taxa that should be added to the recent collection, for historical reasons or "filling the gaps" in the inventory of certain families or genera.

After the booklet of HeInz (1895-1896), there is a large gap of more than 50 years (until the establishment of the current Garden database in 1948), and throughout the data on the glasshouse collections are missing. Some data on the glasshouse-plants can be found in the gardeners' working diaries (their manuscripts since 1926 are placed in the Botanical Garden archives), but never in the form of complete or thorough plant lists. REGUlA (1997) supposes that a comprehensive Garden database did not exist in the first half of the $20^{\text {th }}$ century - however, this is hard to believe, as every European botanic garden keeps a register of their inventory in one form or another, sometimes for centuries. Moreover, we are in possession of a single preserved notebook from 1898 (Figs. 5ab) containing a list of conifers in the Garden, which implies that there must have been something similar for at least some of the other collections. It could be assumed with some certainty that the Garden collections were in the first half of $20^{\text {th }}$ century at least twice seriously impoverished (during the First and the Second World War), but also that there were some longer periods of thriving. Whatever the case, comprehensive data for the whole period are sadly missing.

Recent Garden records (Figs. 6ab) were founded in 1948, with the arrival of the first post-WWII director of the Garden, Dr. Sala Ungar (1908-1988). It is since then that we can track the contents of the collections in detail, and botanists of the Garden have kept up this activity. In addition, it is since 1948 that every potted plant in the Garden's greenhouse-facilities has been labelled with its scientific name, town/botanical garden of origin, year of acquiring and the serial number of the Garden-database, so the tracking of the collections is much easier. 
The Botanic Garden database holds approx. 28,000 cards (single entries for a single taxon, Figs. 6ab), which are divided in two parts: what is called the passive database (plants not present in the Garden, but grown during the years) and the active database (plants recently grown in the Garden), which was digitalized in 2002 as a component of the Flora Croatica Database with restricted access (http://hirc.botanic.hr/fcd/crofloradb/, Fig. 8). The contents of the passive and active card database are connected with several notebooks (Fig. 5b), listing the plant names of all received species in several groups (native plants, exotic plants, annuals), arranged by their serial numbers.

Cards (Figs. 7ab) were designed to carry vital data on each plant entering the collection: from the scientific and common names, through the city and year of acquiring, to practical notes on cultivating and managing the species. Valid scientific names of the time were occasionally unconfirmed or doubtful, due to the lack of adequate literature: for example, some names were stated only according to some Delectus Seminum. For most of the species already living in the glasshouses when the new database was launched, the oldest cards state just "plant found growing in the Garden", without any further data on origin etc., which were obviously unknown: for example, Lunularia cruciata (nn, 1949), Isoloma pictum (nn, 1950 - Figs. 7ab), Callisia repens (nn, 1950), Selaginella involvens (nn, 1950), Streptocarpus rexii (nn, 1950), etc. ("nn" is short for "unknown origin").

For all newcomers after 1948, the local area or the botanic garden of plant seed/cutting/ bulb origin was noted, together with a year when the material was acquired: the oldest plant in the temperate collection recorded in such a manner was one Dracaena 'Haageana', obtained from Palermo (Italy) in 1948. The facility or an outdoor-place where the plant was cultivated in the Garden was also noted: for example," $S$ " for a greenhouse (without further notice of which one exactly), " $\mathrm{P}$ " for arboretum, " $\mathrm{Y}$ " for nursery, etc.

Initially, a collection inventory was kept annually, when the status of each specimen was carefully noted in the card of each taxon: for example, the number of potted, replanted, sold or died-off specimens; newly grown specimens by seeds, cuttings or by dividing of the old ones, etc. For many taxa the data are incomplete, and for the minority it was noted only that they were successfully germinated and placed in the temperate collection, but nothing further. The reason for this may lay in the fact that during the years and even decades when the Garden staff and finances were limited, sometimes five or more years would pass between two inventories. Also, from some cards, it is obvious that the Garden staff struggled to grow certain interesting species in different conditions, year after year, but failed, probably due to the unfamiliar conditions under which some plant should be germinated or cultivated. It is also interesting to see that via Delectus Seminum publications the Garden obtained some species from native seeds, collected in remote regions of the world: for example, one Cycas taiwaniana, today listed in CITES' Appendix II (Anonymous, 1979), as "Trade controlled to avoid use incompatible with species survival - Global".). In places with a high rate of endemism (South Africa, Australia, Madagascar, China, Pacific Islands...) collecting in the wild is nowadays strictly forbidden.

Tab. 2 (not shown here; available in the Supplement of the electronic version of this article) comprises the details extracted from the passive Garden-database, contained in the cards of individual taxa grown in the warm greenhouse conditions since 1948, but now missing in the recent temperate collection (nonetheless, some are growing in the other parts of the Garden). After the digital Garden-database was established in 2002 (http://hirc.botanic.hr/fcd/crofloradb/), this "silent" catalogue of approximately 20,000 
entries has rarely been consulted: during this study, some of the cards were extracted for the first time in decades after they were removed from the active database of living plants. In examination of this part of the database I found some very interesting data, as well as many synonyms and double (even triple) entries for the same plant sample or species. Most of the older passive database cards do not carry precise information on the greenhouse-conditions in which some plant lived, just a symbol "S" ("staklenik", a glasshouse in Croatian). In such cases, again, I had to make educated guesses so it is possible that I either overlooked some plant species which should be placed in the temperate (warm) glasshouse collection, or inventoried some that were never actually grown in such conditions. It is only since 1985, when the new "Aroideae" glasshouse was erected, that the cards have carried the "S8"-sign, depicting that some particular plant lives in the temperate glasshouse collection Number 8 - which is the "Aroideae". Also, it is worth mentioning that during my inspection of the passive database I have noticed several larger plant die-offs in the glasshouses - perhaps due to some disease or vermin, when the pest-control was insufficient. Unfortunately, there were some periods of time after WWII when even the basic care of the Garden was deficient: times with a severe lack of finances and trained staff - even in the recent era, during the Croatian War of Independence (19911995), when just the minimal care for plants could have been provided.

The final part of this study is based on our active Garden-database and recent records on the plants growing in the warm glasshouse, concluding with May $30^{\text {th }}, 2015$. Today, the inventories of the glasshouse collections are made biannually, when the new nomenclature is obtained, as the scientific names of plants often change according to the latest research on the origin and relations of species. A paradox is that, in contrast to the shortage of proper literature experienced during the olden days, today we are overwhelmed with information. Various authorities disagree greatly on the valid nomenclature and synonymy of plants (even in the proper name(s) of the author(s) of species, not even counting the accepted names of taxa), so we decided during the 2000s to consult the Tropicos database of the United States' St. Louis Missouri Botanical Garden (http://www. tropicos.org/), to obtain the recent valid scientific names of exotic, non-Croatian and non-European native species growing in the glasshouses. During the last several years, if Tropicos lacks information, the Plant List database (http://www.theplantlist.org/) has been consulted, followed by the other on-line sources, such as the International Plant Names Index (http://www.ipni.org/), etc.

Tab. 3 depicts plants that live in the warm, "Aroidea"-glasshouse today. The basis of that list is my own register of inventories, assembled biannually since 2000 by the attending gardeners in the temperate greenhouse collection. Besides, the active Garden database was thoroughly examined and compared with the data contained in the digitalized database (http://hirc.botanic.hr/fcd/crofloradb/), during which many cards ought to be removed to the passive part of the records. We are still keeping track of the origin of plant material and the precise place in the Garden where each specimen is placed. Most of the plants in the recent temperate collection are represented with three to five claypotted-specimens, which are rejuvenated (vegetatively or germinatively) when possible. New plants for this collection are obtained through the Delectus Seminum network of inter-botanic-garden seed exchange, and grown in the Garden quarantine facilities. Rarely, some gardens offer living plants (bulbs, cuttings, plantlets) from which the growing is faster, if these vegetative fragments survive transport and frequent delays at the custom-offices. From time to time, some interesting plants (primarily cultivars that are impossible to grow from seeds) are purchased from nurseries or garden centres. 


\section{RESULTS \& DISCUSSION}

Tab. 4 depicts the relations in estimated numbers of families, genera and taxa living in the temperate glasshouse collection of the Botanical Garden (Faculty of Science, University of Zagreb) during the years.

Tab. 4. Compilation of numbers of families, genera and taxa for the five large groups of temperate glasshouse collection plants (Thallose liverworts, Spikemosses, Ferns, Dicotyledons and Monocotyledons), extracted from three sources: 1895 - the booklet written by HeInz (1895-1896); 1948-2014 - the passive Garden database on the plants grown during that period, but recently not existing in the temperate glasshouse collection; 2015 - the active Garden database on the plants recently grown in the temperate glasshouse collection. An asterisk $\left(^{*}\right)$ depicts an estimated number, due to the lack of precise data.

\begin{tabular}{|c|c|c|c|c|}
\hline Plant group & Year(s) & Families & Genera & Taxa \\
\hline \multirow{3}{*}{ Thallose liverworts } & 1895 & 1 & 3 & $5^{*}$ \\
\hline & 1948-2014 & 6 & 8 & 11 \\
\hline & 2015 & 0 & 0 & 0 \\
\hline \multirow{3}{*}{ Spikemosses } & 1895 & 1 & 1 & $10^{*}$ \\
\hline & 1948-2014 & 1 & 1 & 20 \\
\hline & 2015 & 1 & 1 & 9 \\
\hline \multirow{3}{*}{ Ferns } & 1895 & 6 & 21 & $35^{*}$ \\
\hline & 1948-2014 & 13 & 26 & 53 \\
\hline & 2015 & 7 & 13 & 25 \\
\hline \multirow{3}{*}{ Dicotyledons } & 1895 & 40 & 92 & $130^{*}$ \\
\hline & $1948-2014$ & 38 & 120 & 297 \\
\hline & 2015 & 31 & 72 & 159 \\
\hline \multirow{3}{*}{ Monocotyledons } & 1895 & 14 & 50 & $70^{*}$ \\
\hline & 1948-2014 & 16 & 75 & 152 \\
\hline & 2015 & 17 & 60 & 127 \\
\hline \multirow{3}{*}{ In total } & 1895 & 62 & 167 & $250^{*}$ \\
\hline & 1948-2014 & 74 & 230 & 533 \\
\hline & 2015 & 56 & 146 & 320 \\
\hline
\end{tabular}

As already emphasized, it is hard to compare data from various ages due to the extensive synonymy and the many changes in taxonomic and systematic relationships of plants. For example, once known only as "Hepaticae" (Tab. 1), thallose liverworts were later subdivided into many families (Tab. 2), some monotypic. On the other hand, many previous, smaller families of monocotyledons are now integrated in the large "Asparagaceae" (Tab. 3), etc. As the aim of this study is neither to solve the taxonomical or nomenclatural problems, nor to get involved in the scientific debates on the more or less "natural" subclassifications of the Plant Kingdom, for purely "gardener-friendly" purposes I have provisionally assembled the plants of the Garden's temperate collection into five groups. These are: Thallose liverworts (Marchantias), Spikemosses (Selaginellas), Ferns (Pteridophyta), Dicotyledons ("Dicots", Magnoliopsida) and Monocotyledons ("Monocots", Liliopsida). 
Here follow some explanations and clarifications of the data listed in Tabs. 1, 2 and 3 (in Supplements), arranged by five aforementioned provisional groups of plants formerly or recently grown in the temperate glasshouse collection of our Garden:

\section{Thallose liverworts (Bryophyta-Marchantiopsida-Marchantiales)}

According to the earliest data (HeInz, 1895-1896, pg. 13-14), several taxa in three genera of "Hepaticae" (Tab. 1 \& 4) were grown in the glasshouse in 1895: Riccia ("various"), Marchantia and Lunularia. There are no references to the origin of these liverworts in the early days of the Garden. These primitive plants were always in the first place important for the teaching of botany, but sometimes became troublesome weeds in other plant pots and Garden surfaces: for example, Lunularia vulgaris and Marchantia polymorpha were already infesting the greenhouses in the early 1890s (Heinz, 1895-1896).

Since 1948, 6 liverwort families with 8 genera and 11 species (Tab. 2 \& 4) passed through the warm glasshouses: Aytoniaceae (genera Fimbriaria and Mannia), Corsiniaceae (Corsinia), Lunulariaceae (Lunularia), Marchantiaceae (two species of Marchantia and two of Dumortiera), Targioniaceae (two species of Targionia) and Wiesnerellaceae (Wiesnerella), according to the recent nomenclature. Almost exclusively, the liverworts were obtained from the botanic gardens in Antwerp (Belgium) and Jena (Germany) in the early 1960s, but wilted or were displaced from the collection before 1970.

Recently, only the common liverwort (Marchantia polymorpha) is periodically placed in the temperate glasshouse, when it is needed in larger amounts for the purpose of botany classes or research, but not as a regular part of the temperate greenhouse collection (Tab. $3 \& 4$ ). Besides this, three representatives of thallose liverworts today grow in the Garden (and so outdoors): Lunularia cruciata, Conocephalum conicum and Frullania dilatata (data of Antun Alegro, pers. comm. - unpublished).

\section{Spikemosses (Lycopodiophyta-Isoetopsida-Selaginellales)}

Spikemosses or selaginellas belong to a monotypic family (Selaginellaceae), which contains only a single genus - Selaginella. Although there were some attempts to divide the genus and family into smaller groups, the simple "single family-single genus" approach still prevails.

According to Heinz (1895-1896, pg. 16), "there is a nice collection of many beautiful spikemosses in the glasshouse", without a more concise description of the subordinate taxa: whether he was referring to the number of Selaginella species or to individual specimens (Tab. 1), I cannot tell.

After WWII, as many as 20 spikemoss species with two subordinate taxa went through the temperate glasshouse collection, which are absent today (Tab. $2 \& 4$ ). It seems that Selaginella kraussiana, S. millspaughii and S. stellata were most often ordered from the various Delecti Semini - however, the most favourite botanical garden from which to order these attractive plants was that in Lyon (France), during 1960s. The longest-running specimens in the collection were some of S. kraussiana and S. stellata, living for about 25 years (1962-1988).

Nine species of spikemoss are permanently living in the temperate glasshouse today (Tab. 3 \& 4). Most of them are quite old, originating from the 1960s: for example, our recent $S$. tenuissima was obtained from Lyon (France) in 1951. It is worth mentioning that Tabs. 2 and 3 overlap in two species: Selaginella involvens and S. kraussiana, ordered among others from Cluj-Napoca (Romania) in 2015. The reason for this is simple: the newcomers are not yet fully established after they arrived as plantlets, and in poor shape. 


\section{Ferns (Pteridophyta - Polypodiopsida/Pteridopsida)}

The classification of ferns and their relatives is very difficult and much disputed. In almost every source consulted - printed or accessible on-line - one will find different views on the taxonomy, nomenclature and systematic position of taxa. The synonymy of ferns is enormous, sometimes counting 30-or more "accepted", "valid", "synonymic" and "rejected" names for a single taxon, which could be attributed to several different families. To make it all worse, a confusion around the natural and cultivated varieties ("cultivars"), forms and hybrids tangles the nomenclature furthermore.

According to Heinz (1895-1896; pg. 14-15), "many species" from 21 fern genera and 6 families (Tab. 1 \& 4) of that time grew in the Exhibition Glasshouse in 1895, many of which are today missing. For various taxa just a genus is stated, as "containing several species", so it was impossible to sort out which. It is very impressive to see, just three years after the construction of the Exhibition Greenhouse, so many ferns living in the Garden: even the primitive whisk fern (Psilotum nudum) of the ancient Psilotales, and many other true-fern species (as seen in Tab. 1), which never appeared again.

In Tab. 2 I have listed all ferns grown since WWII in the temperate greenhouse conditions: the list includes 13 recent families, 26 genera and 53 taxa (Tab. 4) which are not present in the nowadays collection. We now miss the representatives of 7 fern families and 10 genera - but this is, actually, disputable. As already stated, different botanical sources classify ferns in different ways: for example, the genus Arachniodes is included in Polystichum, Histiopteris in Pteris, Drynaria in Polypodium etc., so this "lack" in representatives of genera and families is relative.

Recent collection of the temperate glasshouse ferns lacks some species today living in the tropical facilities of the Garden (Platycerium, Ceratopteris). The temperate glasshouse collection is at the moment home to 7 fern families (Tab. 3 \& 4 ) and 13 genera with several dozen specimens from 25 taxa (species, subspecies, cultivars). As the ferns are not easily developed from spores, in ordering taxa from other gardens the living-plants were preferred. Many specimens were obtained in a form of already established plants, just after the WWII often from the Croatian Opeka Arboretum, but also from other gardens and private donations (in Tab. 3 assigned as "nn" - origin unknown; acc. to Dr. Ljerka Regula - pers. cont.). The oldest living fern specimen in our collection originates from plants obtained in 1954: these are well-known ornamentals Pteris multifida and Nephrolepis exaltata (Tab. 2). Always very popular among the visitors, our exotic ferns overwinter indoors, but oversummer in the open: in the Garden fernarium, where they can be closely approached. Potted tropical and subtropical ferns (for example Fig. 9a) are combined in between the permanently planted, open-air ferns of the cooler parts of the world, to form a summertime-long fern collection.

\section{"Dicotyledons" ("dicots", Magnoliopsida)}

Most of the former and recent exotic plants of the Garden collections belong to the large and (also disputed) angiosperm group of "dicotyledons" or "magnoliopsida".

As seen in Tab. 1 (HeInz, 1895-1896), in the late $19^{\text {th }}$ century more than 90 genera from 40 families lived in the temperate glasshouse collection (Tab. 4) - and this is just an estimation. Like today (Tab. 3), the most abundant were the families of Gesneriaceae, Moraceae and Amaranthaceae, out of which many of the listed plants are present in the recent collection. Some scientific names are difficult to confirm (especially because the authors of species-names are missing): for example, genus "Candollea" (fam. Dilleniaceae), or species such as "Uragoga (Cephaelis) Ipecacuanha" (fam. Rubiaceae). 
According to my calculations (Tab. 2 \& 4), during the last 70 years almost 300 "dicot"taxa passed through the temperate glasshouse collection but are now missing, from 120 genera and 38 families. Some of the species are growing in other parts of the Garden (for example Ruellia blumei, Iresine herbstii, etc.), but a large portion was linked to the warmgreenhouse environment exclusively (for example begonias, peperomias, etc.) and are not grown elsewhere. More than 70 now absent taxa of Begoniaceae, 40 of Gesneriaceae, 30 of Piperaceae etc., once lived in the warm greenhouse. Some species were ordered repeatedly (for example Begonia dregei, B. grandis ssp. evansiana, cultivars of Begonia $\mathrm{x}$ tuberhybrida, B. 'Rex Cultorum' or Saintpaulia ionantha; Jacaranda mimosifolia, Tipuana tipu, Clerodendrum speciosissimum, many peperomias, etc.), among which the absolute winner is Streptocarpus rexii: it was ordered a dozen times during the years, but lived briefly (Tab. 2). Also, it is obvious that Delecti Semini of some botanic gardens were more popular than the others: for example the gardens in Besançon, Gent and Nancy (France), Antwerp (Belgium) and Vienna (Austria - Institute, Belvedere and Schönbrunn). For about 50 samples the source is unknown ("nn" in Tab. 2). Some of those plants were received from the Garden staff and visitors, and some were purchased, but without a known origin (acc. to Dr. Ljerka Regula - pers. comm.).

In the current "Aroideae"-glasshouse about 160 "dicots" from 72 genera and 31 families have recently been grown (Tab. $3 \& 4$ ). The most abundant are the families Piperaceae (26 taxa in two genera) and Moraceae (24 taxa in two genera), followed by Acanthaceae (20 taxa in 15 genera, among which Pleuropetalum darwinii is placed in the IUCN Red list), and Gesneriaceae (12 taxa in 6 genera). The Begoniaceae family recently holds 10 taxa (Fig. 9b), while in the past (Tab. 2) it was far more abundant. Around a dozen families hold only a single representative (again, the nomenclature is conducted according to the Tropicos botanic database - other sources might consider the ranges of families and subordinate taxa differently), but most of them are represented in the other (glasshouse) collections (for example, Araliaceae, Oleaceae, Passifloraceae, etc.). Most of the plants grown in the large pots are placed during the summertime in the open, to be reachable to our visitors. To them, the most interesting are the collections of Coffea (many pots, Fig. 9c), Piper (several taxa) and Ficus (more than 20 taxa, Figs. 9de). The Garden glass houses have been closed to the public for almost a century now: in future, when the old Exhibition Glasshouse is reconstructed, part of the temperate collection will gain a space of its own ("topla kuća" - "warm house").

\section{"Monocotyledons" ("monocots", Liliopsida)}

A few of the exotic plants once grown in the temperate-glasshouse collections belong to the angiosperm group of "monocotyledons" or "liliopsida". At the end of the $19^{\text {th }}$ century, Heinz (1895-1896) depicts the representatives of at least 50 genera in 14 families at the time (Tab. 1 \& 4). Members of the Araceae, Bromeliaceae, Liliaceae and Zingiberaceae were most abundant.

According to my calculations (Tab. 2 \& 4), in the temperate-glasshouse collection after 1948 about 150 "monocots" from 75 genera and 16 families once grew but are now missing. Families of Bromeliaceae, Araceae, Commelinaceae and Asparagaceae were most abundant in the temperate greenhouse collection. Most of the representatives of, for example, fam. Orchidaceae and Amaryllidaceae (s.l.) are today cultivated in the other greenhouse collections.

The recent temperate glasshouse collection holds around 130 "monocots" from 60 genera and 16 families (Tab. $3 \& 4$ ). The most abundant are the families Asparagaceae 
(40 taxa in 11 genera) and Bromeliaceae (35 taxa in 14 genera - Billbergia macrolepis is placed in the IUCN Red List). Several families hold only a single representative, but, again, according to other sources, some of them are included in other (larger) families. Some of our temperate collection "monocots" are also placed during the summertime in the open, such as the famous birds of paradise (Strelitzia), the currently large Asparagaceae (dracaenas, sansevierias) and some larger Bromeliaceae (Fig. 9f).

Representatives of other evolutionary groups and families were occasionally placed in the warm greenhouse facilities for various reasons, among which the most often was a simple lack of space. These "ephemerals" are not included in Tab. 2, for they were regularly moved to the other collections after some time. For example, members of the ancient Gnetaceae family (mostly Gnetum gnemon) lived occasionally in the temperate glasshouse, as well as the young plants of the Araucariaceae family (Araucaria angustifolia, A. araucana, A. bidwillii, A. cunninghamii), which spent several years of their "childhood" in the temperate greenhouse collection, before being added to the cool greenhouse collection.

\section{CONCLUSIONS}

According to a thorough examination of the available sources, I found that during the last 125 years at least 1000 plant taxa from 380 genera and 90 families were cultivated in the temperate (warm) glasshouse collection of the Botanical Garden, Faculty of Science, University of Zagreb. As early as 1895 (HeINZ, 1895-1896), at least 250 taxa of 167 genera and 62 families inhabited this collection. After that, until the end of the WWII, data on the glasshouse collections are missing.

The passive part of the recent database, keeping details on plants that are today missing, shows that between 1948 and 2014 at least 533 taxa from 230 genera and 74 families passed through the temperate glasshouse collection.

The active part of the recent database holds information on the plants cultivated in the Garden at the moment: accordingly, the temperate glasshouse plant collection contains 320 taxa from 146 genera and 56 families.

\section{Acknowledgements}

I wish to express my sincere gratitude to the Botanical Garden volunteers Renata Biba, Kristina Blagušević, Anja Gotić and Livia Hamaček, students of biology (Faculty of Science, University of Zagreb), who typed and sorted the vast lists of plants grown in the temperate glasshouses extracted from the Garden database. My appreciation extends to my colleagues, Biserka Juretić, Darko Mihelj and Dr. Vanja Stamenković, who patiently compared these lists with their own; the gardeners Zrinka Dević, Gordana Ljevar and Mirela Ravlić, for constructing the inventories of the recent temperate glasshouse collections since 2000; and Dr. Ljerka Regula, retired manager of our Botanical Garden, for sharing countless stories and unpublished data over the years. I am also thankful for the good will and valuable suggestions of my esteemed peers, Dr. Jože Bavcon, Director of the Ljubljana University Botanical Garden (Slovenia), and Dr. Dario Kremer, Head of the Fran Kušan Pharmaceutical Botanical Garden, Zagreb University (Croatia). Finally, the efforts of all Garden managers, curators and gardeners, in maintaining the collections of living plants in the Botanical Garden of the Zagreb Faculty of Science, since its foundation in 1889 until today, are greatly admired and appreciated - most of all those of its founder, Professor Antun Heinz. 


\title{
REFERENCES
}

Anonymous, 1979: Convention on International Trade in Endangered Species of Wild Fauna and Flora. Available at: http://www.cites.org.

Anonymous, 2007: The Garden History Society, Garden History advanced Horticultural Techniques in Korea: the earliest documented greenhouses, p. 68-84. W.S. Maney and Son, Ltd.

Bavcon, J., MarinčEK, A. \& RavnJAK, B., 2012. Novi tropski rastlinjak/ The New Tropical Greenhouse. In Tropski rastlinjak v Ljubljani / The Tropical Greenhouse in Ljubljana, Botanični vrt, Oddelek za biologijo, Biotehniška fakulteta, Ljubljana, 7-44 pp. /and the literature within/.

Grant, F., 2013: Glasshouses. Shire Library, Westminster, USA, p. 72.

HeInz, A., 1895-1896: Kr. Botanički vrt u Zagrebu. Glasnik Hrvatskoga naravoslovnoga društva, 8(1-6), $1-54$.

Juretić, B., Kovačić, S., Mihelj, D., Sandev, D. \& Stamenković, V., 2014: Fifty Sights at the Botanical Garden: for Passers-by, Strollers and Real Enthusiasts - a Guide to the Botanical Garden, Faculty of Science, University of Zagreb. Botanical Garden, Faculty of Science, Zagreb, p. 110.

Kovačić, S., Sandev, D., Mihelj, D., \& Stamenković, V., 2014: Win some, lose some - Statutorily strictly protected indigenous plant species in the Botanical Garden of the Faculty of Science, University of Zagreb (Croatia). Natura Croatica 23(2), 415-432.

Nikolić, T. (ed.), 2015: Flora Croatica Database (incl. the Botanical Garden Archives, with restricted access; Stamenković, V. (ed.): http://hirc.botanic.hr/fcd/crofloradb/). Faculty of Science, University of Zagreb.

PARIS, H. S. \& JANICK, J., 2008: What the Roman emperor Tiberius grew in his greenhouses? “Cucurbitaceae 2008" - Proceedings of the IXth EUCARPIA meeting on genetics and breeding of Cucurbitaceae (Pitrat, M., ed), p. 33-46. INRA, Avignon (France), May 21-24th, 2008.

Regula, Lj., 1997: Botanički vrt Prirodoslovno-matematičkog fakulteta Sveučilišta u Zagrebu - Spomenica o 100. obljetnici utemeljenja, 1889.-1989. /and the literature within/. Školska knjiga, Zagreb, p. 173.

Sandev, D., Minelj D. \& Kovačić, S., 2013: Meeting Target Eight - Ex situ conservation of Croatian threatened and statutorily protected plant species in the Botanical Garden of the Faculty of Science, University of Zagreb (Croatia). Natura Croatica 22(2), 343-362.

The International Plant Names Index, 2012: Published on the Internet http://www.ipni.org

The Plant List, Version 1.1., 2013: Published on the Internet http://www.theplantlist.org/

Tropicos.org., Missouri Botanical Garden, 2015: http://www.tropicos.org

Woods, M. \& Swartz Warren, A., 1990: Glass Houses: a History of Greenhouses, Orangenies and Conservatories. Aurum Press Ltd., London, p. 216.

\section{SAŽETAK}

\section{Obilje bilja - zbirke Botaničkoga vrta \\ Prirodoslovno-matematičkog fakulteta Sveučilišta u Zagrebu (1): Uresnice toplog staklenika - povijesni pregled}

\author{
S. Kovačić
}

Dvije godine po osnutku Botaničkoga vrta (1889.) današnjeg Biološkog odsjeka Prirodoslovno-matematičkog fakulteta Sveučilišta u Zagrebu, u njemu su dovršeni staklenici (1891.). Najveći, izložbeni staklenik izgrađen je po uzoru na slavni Palmenhaus u bečkome Schönbrunnu te podijeljen na nekoliko manjih prostorija namijenjenih uzgoju uresnih (egzotičnih) biljaka hladnih, umjerenih i tropskih podneblja svijeta ("zdena kuća", "topla kuća", "kuća za palme“...). Već 1899. staklenici su dograđeni (Regula, 1997), čime se dobilo na prostoru za proširenje zbirki. Neposredno prije stotog rođendana Vrta, 1985. godine, na mjestu jednog manjeg staklenika podignut je novi, kolokvijalno nazvan "Aroideae" jer je 
inicijalno bio namijenjen uzgoju pripadnika porodice kozlaca (Araceae). Otada, u tom prostoru od oko $150 \mathrm{~m}^{2}$, prezimljuje i trajno živi velika zbirka biljaka toplih i umjerenih podneblja svijeta, koja je davno prerasla okvire planirane porodice i proširena mnogim drugim vrstama sličnih zahtjeva ("sobne biljke"). Uz 30. obljetnicu toga staklenika i 125. rođendan Botaničkoga vrta počeli smo s temeljitim inventariziranjem zbirki. Prema nadolazećim propisima Europske unije ubrzo će biti potrebno službeno registrirati, između ostaloga, i sve žive zbirke biljaka, kako bi se moglo pratiti njihovo podrijetlo i put od sjemenke do odrasle biljke te spriječilo neovlašteno korištenje i moguća zlouporaba materijala.

Analiziranje zbirki toploga staklenika obuhvatilo je, osim današnje kartoteke biljaka, i jedini sačuvani povijesni opis iz 19. stoljeća, objavljen u knjižici osnivača Botaničkoga vrta, profesora Antuna Heinza ("Kr. Botanički vrt u Zagrebu“, 1895-1896). Iz knjižice doznajemo kako je već 1895 . u staklenicima raslo zapanjujuće obilje bilja: barem 250 svojta iz 167 rodova i 62 porodice samo u zbirci toploga staklenika! Iako podatci koje donosi Heinz nisu potpuni i teško ih je uspoređivati s današnjima, na tom popisu ipak možemo prepoznati mnogobrojne vrste koje i danas uzgajamo u Botaničkom vrtu. Nažalost, tijekom sljedećih pola stoljeća sudbina stakleničkih zbirki posve nam nepoznata. Sve do 1948. - kad prva poslijeratna upraviteljica Botaničkoga vrta PMF-a, dr. sc. Sala Ungar, osniva današnju kartoteku - nemamo gotovo nikakvih podataka o stakleničkim zbirkama Vrta. Teško je vjerovati kako u razdoblju od 50 godina nije postojalo sustavno vođenje popisa biljaka u uzgoju, uobičajeno u svim europskim vrtovima već više stotina godina: iako ne možemo biti sigurni, pretpostavljamo kako su inventarne liste, u kojem god obliku bile vođene, izgubljene tijekom Prvog i/ili Drugog svjetskog rata.

Kartoteka osnovana 1948. danas broji oko 28000 kartica - pojedinačnih unosa svojti uzgajanih u Vrtu tijekom posljednjih gotovo 70 godina. Sastoji se od dva dijela: "pasivnog", s odloženim karticama biljnih vrsta i nižih sistematskih kategorija (uključujući kultivare) koje su tijekom desetljeća uzgajane u Vrtu, a danas manjkaju; te "aktivnog", s karticama biljnih vrsta i nižih sistematskih kategorija koje se trenutačno uzgajaju u Vrtu. Taj je dio kartoteke 2002. godine i digitaliziran, u sklopu on-line "Baze hrvatske flore" /Flora Croatica Database/s ograničenim pristupom.

Analizom rijetko korištenog, "pasivnog" dijela današnje vrtne kartoteke utvrdila sam kako su kroz zbirku toplog staklenika od 1948. do danas prošle najmanje 533 biljne svojte koje nam danas nedostaju, iz 230 rodova i 74 porodice (poneke rastu u drugim vrtnim zbirkama).

Trenutačno (popis zaključen s krajem svibnja 2015.), u zbirci toplog staklenika obitava 320 svojta iz 146 rodova i 56 porodica. Naravno, valja imati na umu da je nomenklatura vezana uglavnom uz jednu referentnu botaničku bazu, "Tropicos" Botaničkog vrta savezne države Missouri u St. Louisu (SAD), koji u Vrtu koristimo za ujednačavanje važećih naziva vrsta od ranih 2000-tih. Konzultacijom drugih botaničkih baza dolazimo često do posve različitih podataka o važećim znanstvenim nazivima, sinonimici, pa i pripadnosti rodovima i porodicama.

Usporedbom triju tablica (zbog dužine sve nisu prikazane uz tiskani tekst) s podatcima iz 1895. te iz današnje "pasivne" i "aktivne" kartoteke, nalazimo kako je od 1891. do danas kroz zbirku toplog staklenika prošlo barem 1000 biljnih svojti iz 380 rodova i 90 porodica. Međutim, potrebno je i na kraju naglasiti kako postoji velika "praznina" u podatcima o stakleničkim zbirkama između 1895. i 1948., te su ti brojevi zasigurno veći.

Prilikom buduće registracije zbirki Botaničkoga vrta svakako je važno temeljito popisati sve postojeće biljne vrste u našim zbirkama. No, također je dobro znati i koje su vrste nekad u Vrtu rasle, a danas nedostaju, kako bismo ih mogli ciljano nadomjestiti. 


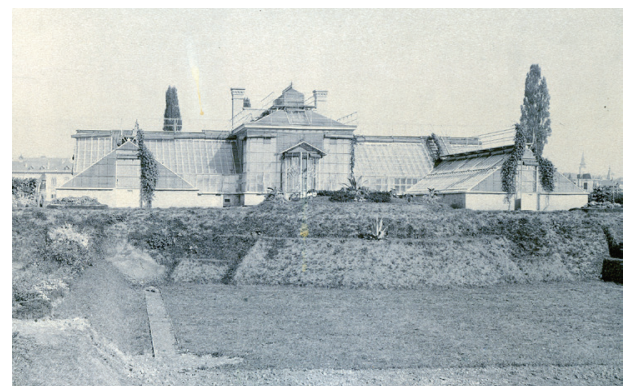

Fig. 1a. The Exhibition Glasshouse in the Botanical Garden shortly after the construction was finished, probably before the time of Heinz's booklet (18951896). The buildings of the Lower City of Zagreb around the Garden are still missing. (Botanical Garden Archives)

Slika 1a. Izložbeni staklenik u Botaničkom vrtu nedugo nakon izgradnje, vjerojatno prije objavljivanja Heinzove knjižice (1895-1896). Zgrada današnjega Donjeg grada nema: parcele uokolo Vrta još su prazne. (arhiv Botaničkog vrta)

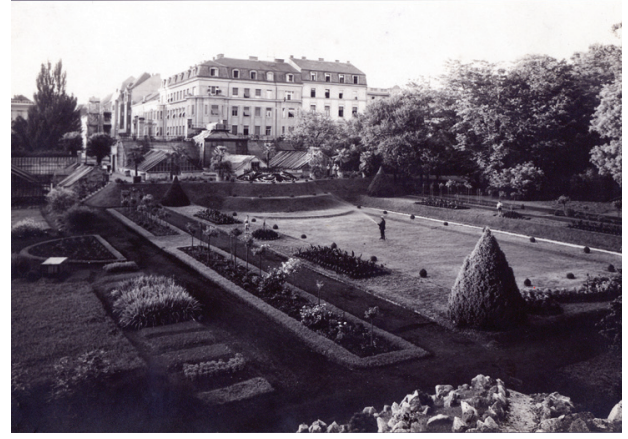

Fig. 1c. Central flower parterre and the Exhibition Glasshouse from the newly erected Karstic rockery (probably 1928): far left, below the Exhibition Glasshouse, is a smaller one (steep roof), at which place since 1985 the "Aroideae" warm glasshouse has stood. (Botanical Garden Archives)

Slika 1c. Pogled na cvjetni parter i izložbeni staklenik s novoosnovane Krške vegetacijske skupine (vjerojatno oko 1928.): sasvim lijevo, ispod razine izložbenog staklenika, vidi se manji, strmoga krova, na mjestu kojega je 1985. izgrađen današnji topli staklenik ("Aroideae"). (arhiv Botaničkog vrta)

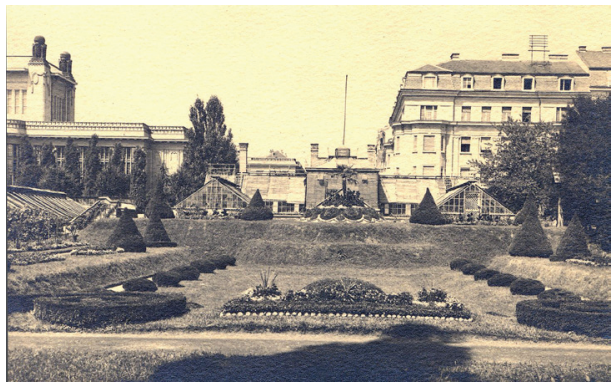

Fig. 1b. The view onto the central flower parterre and the Exhibition Glasshouse before the First World War (probably 1912-1913): it is noticeable that the familiar buildings of the Lower City of Zagreb in the meantime had grown around the Garden. (Botanical Garden Archives)

Slika 1b. Središnji cvjetni parter i izložbeni staklenik prije Prvoga svjetskog rata (vjerojatno 1912.1913.). U međuvremenu su podignute i danas prepoznatljive građevine na Marulićevu trgu: Donji je grad narastao oko Botaničkoga vrta. (arhiv Botaničkog vrta)

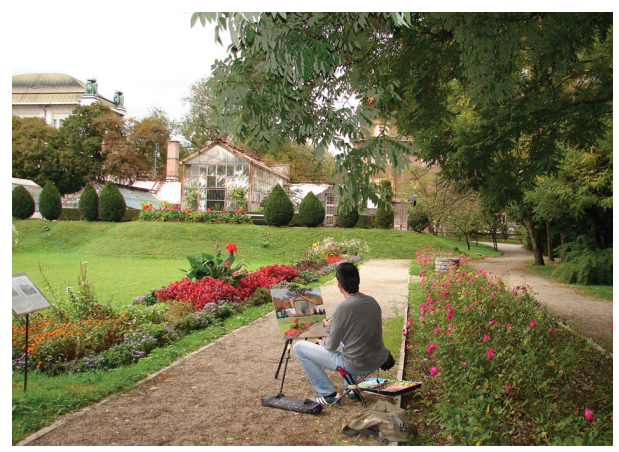

Fig. 1d. Central flower parterre and the Exhibition Glasshouse (in 2005): after a long period of deterioration, this old building today faces the very beginning of its full restoration. (photo by Mirna Kirin)

Slika 1d. Cvjetni parter i izložbeni staklenik 2005. godine. Nakon dugog razdoblja propadanja, stari je staklenik danas napokon pred potpunom obnovom. (fotografija Mirne Kirin) 


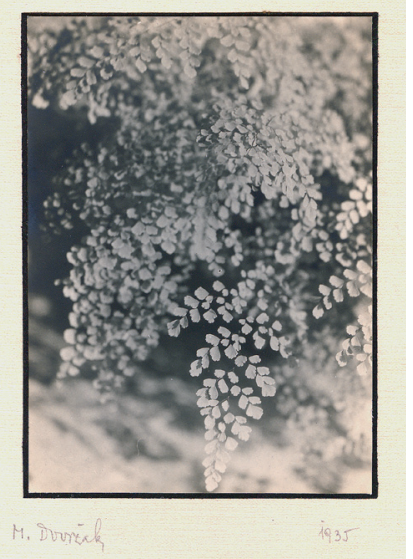

Fig. 2a. A nice example of the temperate collection is a maidenhair fern (Adiantum capillus-veneris), emblem of the Botanical Garden: this photo was taken in the greenhouse in 1935, by Croatian photographer Milan Dvoržak (Botanical Garden Archives)...

Slika 2a. Od samoga osnutka, stalni je stanovnik zbirke toploga staklenika nježna paprat gospin vlasak (Adiantum capillus-veneris), danas logo Botaničkoga vrta. Ovu je fotografiju u stakleniku snimio samoborski fotograf Milan Dvoržak 1935. ... (arhiv Botaničkog vrta)

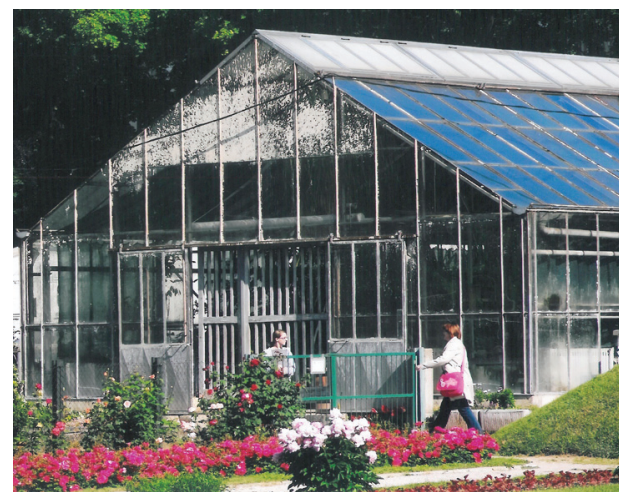

Fig. 3. The "Aroideae" warm glasshouse holds the collection of temperate exotic "houseplants". (photo by Mirna Kirin)

Slika 3. "Aroideae" topli staklenik zatvoren je javnosti kao i svi drugi staklenici u Vrtu. U njemu živi bogata zbirka egzotičnih uresnica, od kojih su mnoge znane "sobne biljke". (fotografija Mirne Kirin)

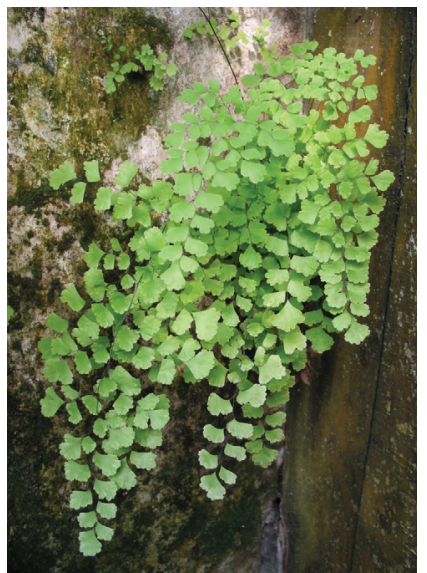

Fig. 2b. ....and this, of the same species in the same place, in 2015 by Dr. Vanja Stamenković, senior Garden curator.

Slika 2b. ... a ovu, iste vrste na istome mjestu, ali 80 godina kasnije (2015.), stručni savjetnik Botaničkoga vrta dr. sc. Vanja Stamenković!

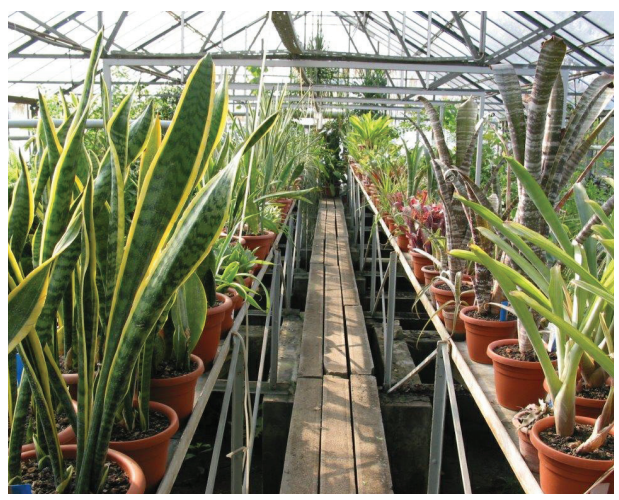

Fig. 4. Recent view of the central part of the temperate glasshouse collection, at "the bridge" over the freshwater pools: on the left, a collection of snake plants (Sansevieria, fam. Asparagaceae); on the right, various Bromeliaceae. (photo by Mirna Kirin)

Slika 4. Današnji pogled na središnji dio toploga staklenika, tzv. "most" iznad bazenčića s kišnicom: lijevo se nalazi zbirka sanseverija (rod Sansevieria, porodica Asparagaceae), a desno raznovrsnih bromelija (porodica Bromeliaceae). (fotografija Mirne Kirin) 


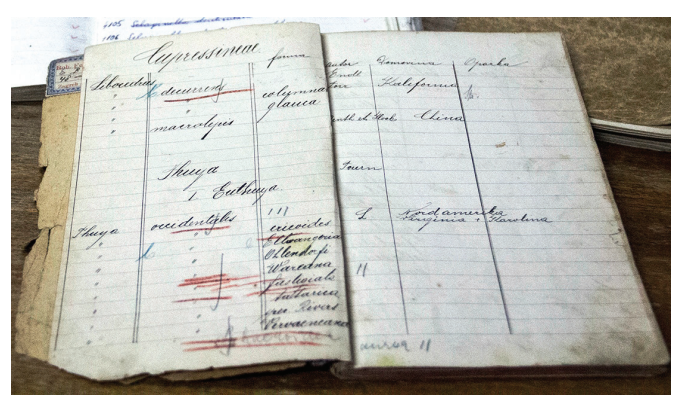

Fig. 5a. The single remaining inventory-notebook from the $19^{\text {th }}$ century ("Coniferae" manuscript, 1898) implies that similar inventories must have existed for the other Garden collections, but were lost during the years. (photo by Vanja Stamenković)

Slika 5a. Jedina sačuvana bilježnica s popisom vrsta u vrtnom uzgoju s kraja 19. stoljeća (zbirka četinjača, "Coniferae", iz 1898.), daje naslutiti kako su slične evidencije postojale i za druge zbirke u Vrtu, no izgubljene su tijekom stoljeća. (fotografija Vanje Stamenkovića)

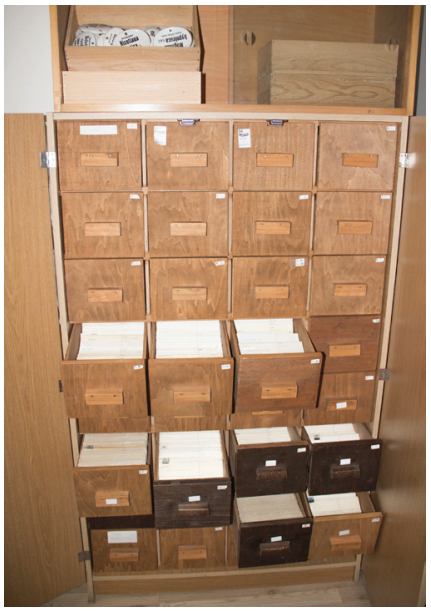

Fig. 6a. The "paper-card" database, established in 1948, consists of 24 compartments. Every compartment contains around 1000 entries, each of a single plant taxon. The lower (open) drawers hold the passive dataset of the plants currently missing from the collections. (photo by Vanja Stamenković)

Slika 6a. Vrtna kartoteka, osnovana 1948., sastoji se od 24 ladice, od koji svaka sadrži i preko 1000 kartica pojedinačnih unosa svojti uzgajanih u vrtnim zbirkama. Donji dio ladica čuva "pasivnu" kartoteku, kartice onih vrsta kojih danas više nema u zbirkama. (fotografija Vanje Stamenkovića)

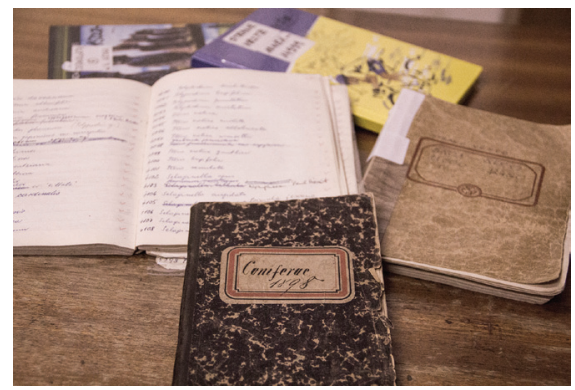

Fig. 5b. Several notebooks connect the passive and active database: all the plants in the collections are arranged numerically and listed in the notebooks. (photo by Vanja Stamenković)

Slika 5b. Nekoliko bilježnica povezuje sadržaje "pasivne" i "aktivne" kartoteke: vrste su u njima popisane po brojevima, pod kojima se vode u kartoteci. (fotografija Vanje Stamenkovića)

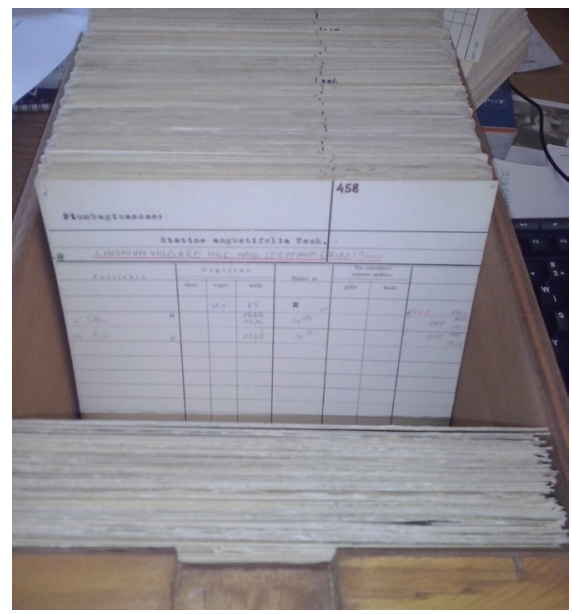

Fig. 6b. A single database compartment, holding the alphabetically ordered cards: active and passive parts are held separately. (photo by Sanja Kovačić)

Slika 6b. Svaka ladica kartoteke sadrži abecednim redom složene kartice pojedinačnih svojti. "Aktivna" i "pasivna" kartoteka čuvaju se odvojeno. (fotografija Sanje Kovačić) 

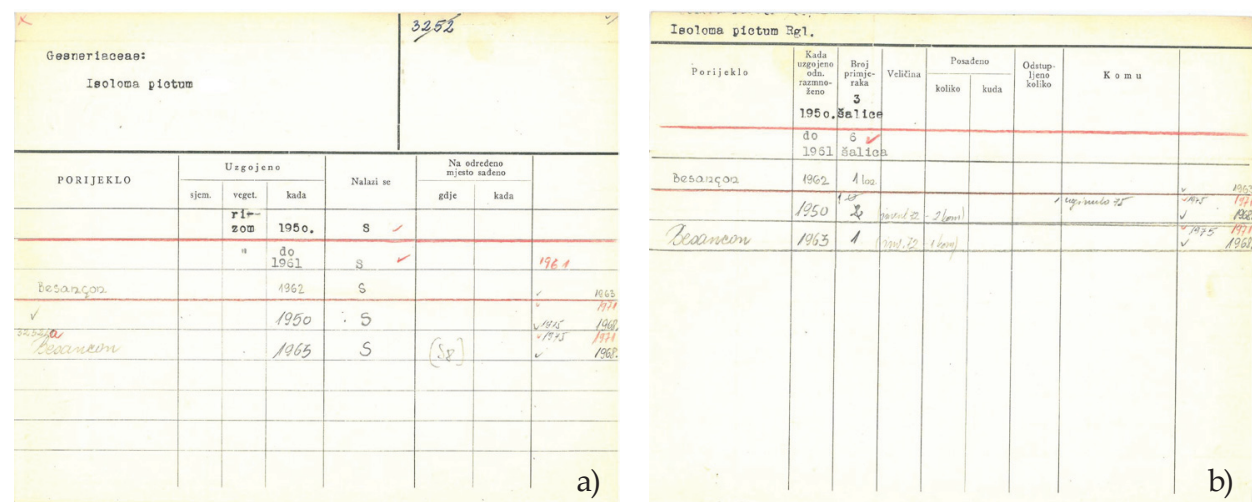

Fig. 7. Card of Isoloma pictum, one of the oldest temperate glasshouse collection-entries in the Garden database: a) front; b) back of the card. (photo by Sanja Kovačić)

Slika 7. Kartica vrste Isoloma pictum, jedne od najstarijih stanarica zbirke toploga staklenika: a) prednja strana kartice; b) stražnja strana kartice. (fotografija Sanje Kovačić)

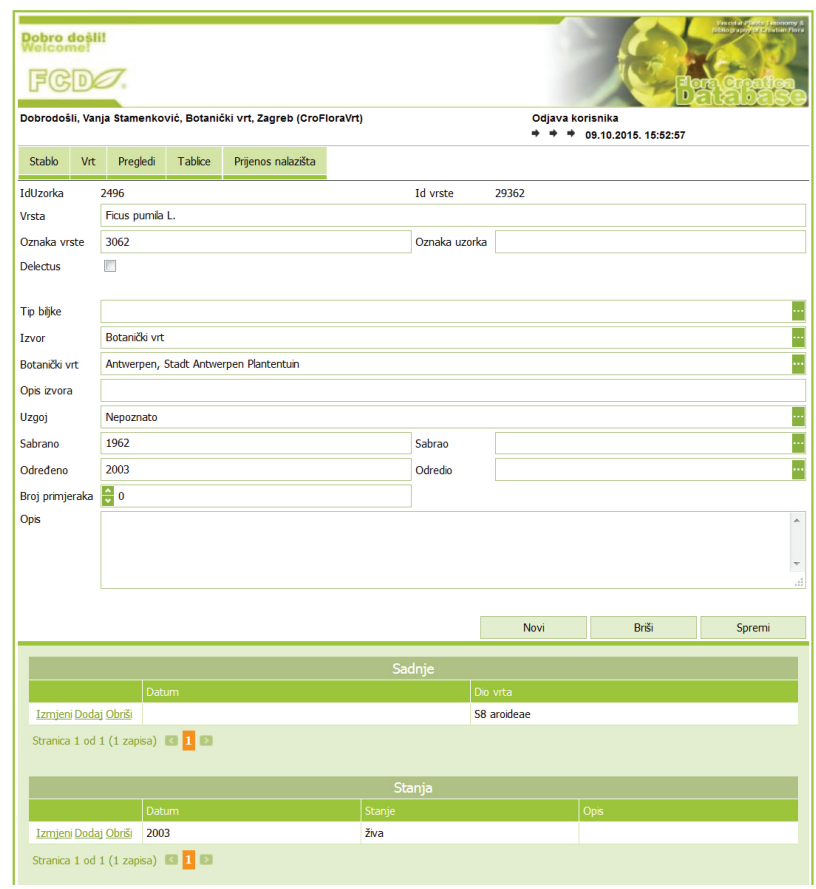

Fig. 8. Recent entry ("card") from the digitalized Garden database. (photo by Vanja Stamenković) Slika 8. Moderna "kartica" digitalizirane vrtne baze podataka, u sastavu Flora Croatica Database. (fotografija Vanje Stamenkovića) 

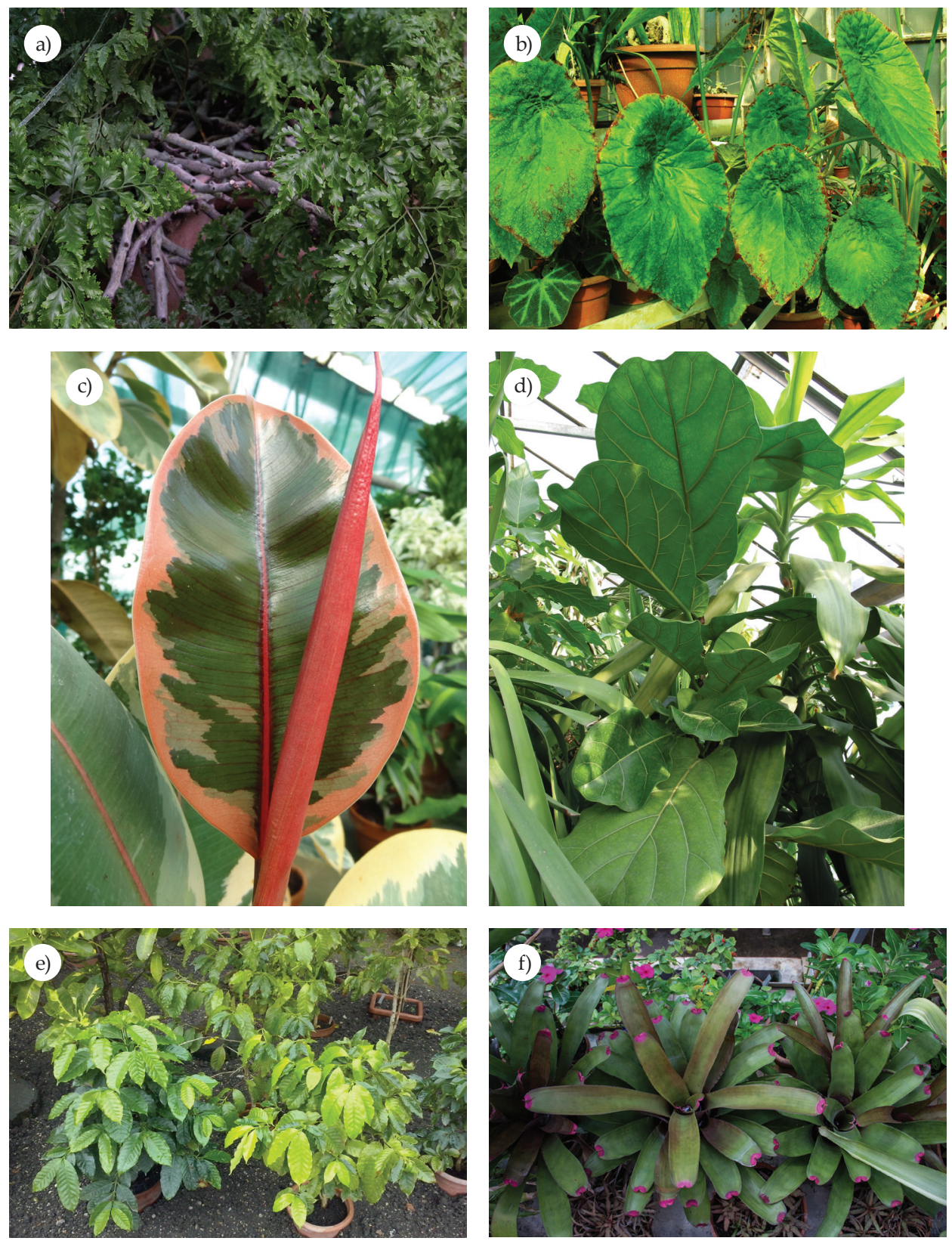

Fig. 9. Several representatives of the recent temperate glasshouse collection: a) Davallia solida 'Superba'; b) Begonia nelumbiifolia c) Ficus elastica 'Belize'; d) Ficus lyrata 'Bambino'; e) collection of Coffea-taxa; f) Neoregelia spectabilis. (photos by Mirna Kirin \& Vanja Stamenković)

Slika 9. Nekoliko predstavnika današnje zbirke toploga staklenika: a) paprat Davallia solida 'Superba'; b) Begonia nelumbiifolia; c) Ficus elastica 'Belize'; d) Ficus lyrata 'Bambino'; e) zbirka kava (Coffea spp.); f) bromelija Neoregelia spectabilis. (fotografije Mirne Kirin i Vanje Stamenkovića) 
Table 1. Estimated collection of temperate (warm) glasshouse in 1895 (Heinz, 1895-1896)

\begin{tabular}{|c|c|c|c|}
\hline Family & $\begin{array}{l}\text { Scientific name (original, acc. } \\
\text { to Heinz) }\end{array}$ & $\begin{array}{l}\text { Still exists } \\
\text { in Garden } \\
\text { collections }\end{array}$ & $\begin{array}{l}\text { Valid \& accepted names, notes and } \\
\text { remarks }\end{array}$ \\
\hline \multicolumn{4}{|c|}{$\begin{array}{l}\text { THALLOSE LIVERWORTS (“Embryophyta } \\
\text { zooidiogama”) }\end{array}$} \\
\hline \multirow{3}{*}{ Hepaticae } & Riccia spp. & yes & several taxa, today fam. Ricciaceae \\
\hline & Marchantia polymorpha & yes & today fam. Marchantiaceae \\
\hline & Lunularia vulgaris & yes & $\begin{array}{l}\text { probably L. cruciata; today fam. } \\
\text { Lunulariaceae }\end{array}$ \\
\hline \multicolumn{4}{|c|}{ SPIKEMOSSES (“Embryophyta zooidiogama”) } \\
\hline Selaginellaceae & Selaginella spp. & yes & \\
\hline \multicolumn{4}{|c|}{$\begin{array}{l}\text { FERNS ("Embryophyta zooidiogama" - } \\
\text { Pterydophyta) }\end{array}$} \\
\hline \multirow{2}{*}{ Cyatheaceae } & Alsophila sp. & no & many synonyms and accepted names \\
\hline & Dicksonia spp. & no & today fam. Dicksoniaceae \\
\hline \multirow{2}{*}{ Hymenophyllaceae } & Hymenophyllum tunbridgense & no & also H. rugosum; many synonyms \\
\hline & Trichomanes radicans & no & several synonyms \\
\hline Marattiaceae & Angiopteris longifolia & no & unresolved name, maybe a cultivar? \\
\hline Osmundaceae & Todea sp. & no & \\
\hline \multirow{18}{*}{ Polypodiaceae } & Blechnum brasiliense & no & $\begin{array}{l}\text { several synonyms; today fam. } \\
\text { Blechnaceae }\end{array}$ \\
\hline & Lomaria Gibba & no & $\begin{array}{l}\text { unresolved name, maybe a cultivar? } \\
\text { today fam. Blechnaceae }\end{array}$ \\
\hline & Pteris longifolia & no & syn. P. vittata; today fam. Pteridaceae \\
\hline & Pteris serrulata & yes & $\begin{array}{l}\text { syn. P. dentata \& P. multifida; today fam. } \\
\text { Pteridaceae }\end{array}$ \\
\hline & Pteris cretica & yes & \multirow{2}{*}{ many synonyms; today fam. Pteridaceae } \\
\hline & Adiantum capillus-veneris & yes & \\
\hline & Adiantum cuneatum & yes & syn. A. raddianum; today fam. Pteridaceae \\
\hline & Adiantum Farleyense & no & $\begin{array}{l}\text { most probably A. tenerum Sw. } \\
\text { 'Farleyense'; today fam. Pteridaceae }\end{array}$ \\
\hline & Adiantum trapeziforme & no & many synonyms; today fam. Pteridaceae \\
\hline & Asplenium spp. & yes & today fam. Aspleniaceae \\
\hline & Gymnogramme spp. & no & $\begin{array}{l}\text { rejected genus-name; today either } \\
\text { Eriosorusor Hemionitis in fam. Pteridaceae }\end{array}$ \\
\hline & Scolopendrium hybridum & no & $\begin{array}{l}\text { "from Lošinj island" (Croatia, endemic); } \\
\text { today Asplenium h., fam. Aspleniaceae }\end{array}$ \\
\hline & Nephrodium sp. & no & today fam. Dryopteridaceae \\
\hline & Polypodium spp. & yes & \\
\hline & Platycerium alcicorne & yes & several synonyms \\
\hline & Ceratopteris thalictroides & yes & many synonyms; today fam. Pteridaceae \\
\hline & Didimochlaena sp. & no & $\begin{array}{l}\text { Didymochlaena; today fam. } \\
\text { Dryopteridaceae }\end{array}$ \\
\hline & Davallia sp. & yes & today fam. Davalliaceae \\
\hline
\end{tabular}




\begin{tabular}{|c|c|c|c|}
\hline & Chrysodium crinitum & no & $\begin{array}{l}\text { unresolved name, maybe a cultivar? } \\
\text { today fam. Pteridaceae }\end{array}$ \\
\hline & "and many others" & yes & family is today divided to many smaller \\
\hline Schizeaceae & Aneimia Phyllitidis & no & $\begin{array}{l}\text { Anemia phyllitidis; several synonyms; } \\
\text { today fam. Anemiaceae }\end{array}$ \\
\hline \multicolumn{4}{|c|}{$\begin{array}{l}\text { DICOTYLEDONAE ("Embryophyta siphonogama" } \\
\text { - Angiospermae) }\end{array}$} \\
\hline \multirow{7}{*}{ Acanthaceae } & Thunbergia spp. & yes & \\
\hline & Aphelandra sp. & no & \\
\hline & Sanchezia sp. & yes & \\
\hline & Ruellia spp. & yes & many synonyms \\
\hline & Fittonia sp. & yes & \\
\hline & Strobilanthes sp. & yes & more than 20 genus-synonyms \\
\hline & "and others" & yes & \\
\hline \multirow{3}{*}{ Amarantaceae } & Achyranthes sp. & no & Amaranthaceae \\
\hline & Iresine $\mathrm{sp}(\mathrm{p})$. & yes & several synonyms; Amaranthaceae \\
\hline & Alternanthera $\mathrm{sp}(\mathrm{p})$. & no & Amaranthaceae \\
\hline \multirow{2}{*}{ Anonaceae } & Anona spp. & no & Annona; Annonaceae \\
\hline & Asimina triloba & yes & Annonaceae \\
\hline \multirow{2}{*}{ Apocynaceae } & Thevetia neriifolia & yes & $\begin{array}{l}\text { accepted name is Th. peruviana; many } \\
\text { synonyms }\end{array}$ \\
\hline & "also other species" & yes & \\
\hline \multirow[b]{2}{*}{ Araliaceae } & Aralia spp. & yes & several synonyms \\
\hline & Fatsia papyrifera & no & $\begin{array}{l}\text { accepted name is also Tetrapanax } \\
\text { papyrifer; several synonyms }\end{array}$ \\
\hline \multirow{4}{*}{ Aristolochiaceae } & Aristolochia elegans & yes & $\begin{array}{l}\text { valid name is } A \text {. littoralis; several } \\
\text { synonyms }\end{array}$ \\
\hline & Aristolochia fimbriata & no & several synonyms \\
\hline & Aristolochia ornithocephala & no & also $A$. labiata; several synonyms \\
\hline & Aristolochia spp. & yes & \\
\hline Asclepiadaceae & Stephanotis floribunda & yes & $\begin{array}{l}\text { several accepted names, incl. Marsdenia f.; } \\
\text { today fam Apocynaceae }\end{array}$ \\
\hline \multirow[t]{2}{*}{ Balsaminaceae } & Impatiens Sultani & no & $\begin{array}{l}\text { accepted name is I. walleriana; several } \\
\text { synonyms, incl. I. balsamina }\end{array}$ \\
\hline & Impatiens spp. & yes & \\
\hline Begoniaceae & Begonia spp. & no & \\
\hline Bignoniaceae & Bignonia spp. & yes & \\
\hline Bombaceae & Durio zibethinus & no & today fam. Malvaceae \\
\hline Candolleaceae & Stylidium adnatum & no & today fam. Stylidaceae \\
\hline Caricaceae & Carica Papaya & yes & $\begin{array}{l}\text { C. papaya, accepted also Papaya carica; } \\
\text { more than } 30 \text { synonyms }\end{array}$ \\
\hline Chenopodiaceae & $\begin{array}{l}\text { "many species also in the } \\
\text { greenhouses" }\end{array}$ & yes & today fam. Amaranthaceae \\
\hline Compositae & "many species in glasshouses" & yes & today fam. Asteraceae \\
\hline Convolvulaceae & Calonyction speciosum & no & $\begin{array}{l}\text { accepted name is Ipomoea alba; many } \\
\text { synonyms }\end{array}$ \\
\hline
\end{tabular}




\begin{tabular}{|c|c|c|c|}
\hline & Pharbitis hispida & yes & $\begin{array}{l}\text { accepted name is Ipomoea purpurea; many } \\
\text { synonyms }\end{array}$ \\
\hline & Quamoclit spp. & yes & $\begin{array}{l}\text { accepted name is also Ipomoea; many } \\
\text { synonyms }\end{array}$ \\
\hline & Mina lobata & yes & $\begin{array}{l}\text { accepted names are also Ipomoea } l \text {. and } \\
\text { Quamoclit l.; many synonyms, incl. } \\
\text { Ipomoea lobata }\end{array}$ \\
\hline & Ipomoea spp. ("many") & yes & $\begin{array}{l}\text { vast spectrum of accepted names and } \\
\text { synonyms }\end{array}$ \\
\hline & "and many more lianas" & no & \\
\hline Cucurbitaceae & $\begin{array}{l}\text { "many species in the } \\
\text { greenhouses" }\end{array}$ & yes & \\
\hline Erythroxylaceae & Erythroxylon Cocca & yes & Erythroxylum coca, two synonyms \\
\hline \multirow{7}{*}{ Euphorbiaceae } & Phyllanthus spp. & no & $\begin{array}{l}\text { approx } 50 \text { genus-synonyms! Today fam. } \\
\text { Phyllanthaceae }\end{array}$ \\
\hline & Daleschampsia sp. & no & Dalechampia; several genus-synonyms \\
\hline & Acalypha spp. & no & many genus-synonyms \\
\hline & Manihot spp. & no & several genus-synonyms \\
\hline & Jatropha sp. & yes & many genus-synonyms \\
\hline & Alchornea (Coelebogyne) ilicifolia & no & $\begin{array}{l}\text { many synonyms of both genera; } \\
\text { sometimes considering as separate }\end{array}$ \\
\hline & $\begin{array}{l}\text { "more species in the } \\
\text { greenhouses" }\end{array}$ & yes & \\
\hline \multirow{11}{*}{ Gesneraceae } & Streptocarpus spp. & yes & fam. Gesneriaceae \\
\hline & Aeschynanthus sp. & yes & syn. Trichosporum; fam. Gesneriaceae \\
\hline & Didymocarpus sp. & no & $\begin{array}{l}\text { accepted name is also Petrocodon; many } \\
\text { synonyms; fam. Gesneriaceae }\end{array}$ \\
\hline & Chirita sp. & no & 3 synonyms; fam. Gesneriaceae \\
\hline & Gloxinia sp. & yes & \multirow{4}{*}{ fam. Gesneriaceae } \\
\hline & Achimenes sp. & yes & \\
\hline & Naegelia sp. & no & \\
\hline & Tydaea sp. & no & \\
\hline & Gesnera sp. & no & Gesneria; fam. Gesneriaceae \\
\hline & $\begin{array}{l}\text { Sinningia speciosa ("Gloxinia of } \\
\text { the gardeners") }\end{array}$ & yes & syn. Gloxinia s.; fam. Gesneriaceae \\
\hline & "and others" & yes & fam. Gesneriaceae \\
\hline \multirow[b]{2}{*}{ Hydrophyllaceae } & Wigandia caracasana & no & valid name is $W$. urens; fam. Boraginaceae \\
\hline & $\begin{array}{l}\text { "and many others, mostly } \\
\text { ornamentals" }\end{array}$ & yes & today fam. Boraginaceae \\
\hline Labiatae & Pogostemon Patschuli & no & $\begin{array}{l}\text { P. patchouli; accepted also P. cablin; several } \\
\text { synonyms; today fam. Lamiaceae }\end{array}$ \\
\hline \multirow{5}{*}{ Leguminosae } & Tamarindus indica & no & several synonyms; fam. Fabaceae \\
\hline & Bauhinia spp. & yes & \multirow{2}{*}{ fam. Fabaceae } \\
\hline & Carmichaelia sp. & no & \\
\hline & Desmodium gyrans & no & several accepted names; fam. Fabaceae \\
\hline & Amicia zygomeris & no & $\begin{array}{l}\text { syn. Hedysarum grandiflorum, fam. } \\
\text { Fabaceae }\end{array}$ \\
\hline
\end{tabular}




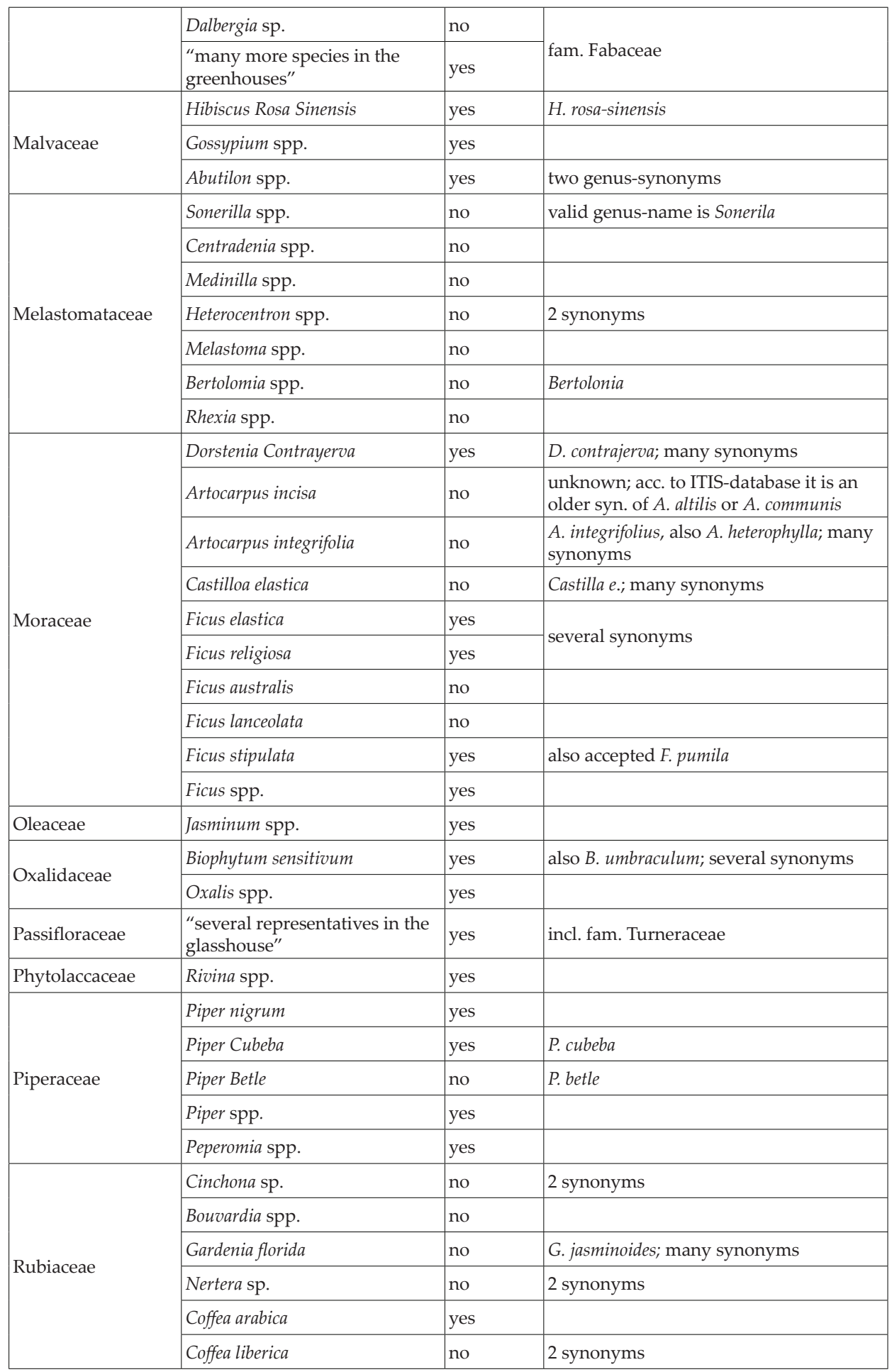




\begin{tabular}{|c|c|c|c|}
\hline & Uragoga (Cephaëlis) Ipecacuanha & no & $\begin{array}{l}\text { U. ipecacuanha; unresolved; several } \\
\text { synonyms and accepted names, incl. } \\
\text { Carapichea i. and Psychotria i.; also } \\
\text { Cephaelis ipecacuanha }\end{array}$ \\
\hline & "and others" & yes & \\
\hline Rutaceae & Pilocarpus pennatifolius & no & many synonyms \\
\hline \multirow{2}{*}{ Scrophulariaceae } & Tetranema mexicanum & yes & $\begin{array}{l}\text { accepted name also T. roseum; several } \\
\text { synonyms; fam. Plantaginaceae }\end{array}$ \\
\hline & "many others" & yes & today fam. Plantaginaceae \\
\hline \multirow{3}{*}{ Solanaceae } & Cestrum spp. & yes & several genus-synonyms \\
\hline & Brunfelsia spp. & yes & \\
\hline & "many others" & yes & \\
\hline \multirow{3}{*}{ Sterculiaceae } & Theobroma Cacao & yes & many synonyms; today fam. Malvaceae \\
\hline & Hermannia spp. & no & today fam. Malvaceae \\
\hline & Dombeya (Astrapaea) sp. & yes & $\begin{array}{l}\text { several genus-synonyms, among which } \\
\text { also Astrapaea; today fam. Malvaceae }\end{array}$ \\
\hline Turneraceae & $\begin{array}{l}\text { "several representatives in the } \\
\text { glasshouse" }\end{array}$ & yes & today fam. Passifloraceae \\
\hline \multirow{3}{*}{ Urticaceae } & Laportea Gigas & no & L. gigas \\
\hline & Boehmeria nivea & no & several synonyms \\
\hline & Pilea spp. & yes & \\
\hline \multirow{2}{*}{ Verbenaceae } & Clerodendron spp. & yes & $\begin{array}{l}\text { valid genus-name is Clerodendrum; fam. } \\
\text { Lamiaceae }\end{array}$ \\
\hline & Lippia citriodora & yes & $\begin{array}{l}\text { valid name is Aloysia c.; several } \\
\text { synonyms }\end{array}$ \\
\hline Vitaceae & $\begin{array}{l}\text { Cissus spp. ("with variegated } \\
\text { leaves") }\end{array}$ & yes & several genus-synonyms \\
\hline \multicolumn{4}{|c|}{$\begin{array}{l}\text { MONOCOTYLEDONAE ("Embryophyta } \\
\text { siphonogama" - Angiospermae) }\end{array}$} \\
\hline \multirow{5}{*}{ Amaryllidaceae } & Sprekelia formosissima & yes & several synonyms \\
\hline & Fourcroya gigantea & yes & $\begin{array}{l}\text { syn. of Furcraea foetida, F. gigantea or } \\
\text { Agave foetida (all valid) }\end{array}$ \\
\hline & Beschorneria yuccoides & no & today fam. Asparagaceae \\
\hline & Curculigo recurvata & no & $\begin{array}{l}\text { syn. Molinieria } r \text {; today fam. } \\
\text { Hypoxidaceae }\end{array}$ \\
\hline & "and other" & yes & $\begin{array}{l}\text { (today many smaller families belong to } \\
\text { Amaryllidaceae) }\end{array}$ \\
\hline \multirow{9}{*}{ Araceae } & Pothos spp. & yes & several synonyms \\
\hline & Anthurium Scherzerianum & yes & A. scherzerianum \\
\hline & Anthurium radicans & no & \\
\hline & Anthurium Andreanum & yes & A. andreanum \\
\hline & Anthurium crystallinum & no & \\
\hline & Anthurium Warocqueanum & yes & $\begin{array}{l}\text { A. warocqueanum T. Moore or A. } x \\
\text { warocqueanum }\end{array}$ \\
\hline & Aglaonema sp. & yes & \\
\hline & Philodendron spp. & yes & several synonyms \\
\hline & Remusatia vivipara & yes & several synonyms \\
\hline
\end{tabular}




\begin{tabular}{|c|c|c|c|}
\hline & Typhonium divaricatum & no & several synonyms \\
\hline & Sauromatum spp. & yes & \\
\hline & Ariopsis peltata & yes & \\
\hline \multirow{10}{*}{ Bromeliaceae } & Nidularium spp. & yes & \\
\hline & Cryptanthus zonatus & yes & \\
\hline & Ananas sativus & no & syn. A. comosus \\
\hline & Bilbergia spp. & yes & \\
\hline & Aechmea spp. & yes & \\
\hline & Pitcairnia spp. & yes & \\
\hline & Dyckia spp. & yes & \\
\hline & Tillandsia spp. & yes & \\
\hline & Vriesea spp. & yes & \\
\hline & $\begin{array}{l}\text { "and many others of the same } \\
\text { family" }\end{array}$ & yes & \\
\hline \multirow{4}{*}{ Commelinaceae } & $\begin{array}{l}\text { Zebrina pendula (Tradescantia } \\
\text { zebrina) }\end{array}$ & yes & both valid; several synonyms \\
\hline & Palisota Barteri & no & Palisota barteri \\
\hline & Dichorisandra sp. & no & \\
\hline & Rhoeo discolor & yes & $\begin{array}{l}\text { accepted names include } R h \text {. spathacea, } \\
\text { Tradescantia spathacea and T. discolor; } \\
\text { several synonyms }\end{array}$ \\
\hline Dioscoreaceae & $\begin{array}{l}\text { Testudinaria Elephantipes } \\
\text { ("wilted") }\end{array}$ & no & T. elephantipes; also Dioscorea e. \\
\hline Gramineae & "several in the greenhouses" & yes & today fam. Poaceae \\
\hline Iridaceae & $\begin{array}{l}\text { "many species in the } \\
\text { greenhouses" }\end{array}$ & yes & (today is family devided) \\
\hline \multirow{12}{*}{ Liliaceae } & Chlorophytum Sternbergianum & no & $\begin{array}{l}\text { Ch. sternbergianum; today fam. } \\
\text { Asparagaceae }\end{array}$ \\
\hline & Bowiea volubilis & yes & \multirow{7}{*}{ today fam. Asparagaceae } \\
\hline & Dasylirion spp. & yes & \\
\hline & Cordyline spp. & yes & \\
\hline & Dracaena draco & yes & \\
\hline & Dracaena spp. & yes & \\
\hline & Aspidistra elatior & yes & \\
\hline & Ophiopogon sp. & yes & \\
\hline & Sanseviera sp. & yes & $\begin{array}{l}\text { valid genus-name is Sansevieria; fam. } \\
\text { Asparagaceae }\end{array}$ \\
\hline & Lapageria rosea & no & today fam. Philesiaceae \\
\hline & Aletris sp. & no & $\begin{array}{l}\text { today in genus Hosta, fam. Asparagaceae } \\
\text { (? acc. to other sources, valid genus of } \\
\text { Nartheciaceae family) }\end{array}$ \\
\hline & Smilax medica & yes & today Smilax aristolochiifolia \\
\hline \multirow[b]{2}{*}{ Marantaceae } & Maranta arundinacea & no & many synonyms \\
\hline & Phrynium sp. & no & $\begin{array}{l}\text { accepted is also genus-name Saranthe; } \\
\text { several synonyms }\end{array}$ \\
\hline Musaceae & Strelitzia spp. & yes & today fam. Strelitziaceae \\
\hline
\end{tabular}




\begin{tabular}{|c|c|c|c|}
\hline Orchidaceae & $\begin{array}{l}\text { "many species in the } \\
\text { greenhouses" }\end{array}$ & yes & \\
\hline \multirow{2}{*}{ Pandanaceae } & Pandanus utilis & yes & many synonyms \\
\hline & "and other similar species" & yes & \\
\hline Taccaceae & Tacca pinnatifida & no & $\begin{array}{l}\text { accepted also T. leontopetaloides; many } \\
\text { synonyms; today fam. Dioscoreaceae }\end{array}$ \\
\hline \multirow{10}{*}{ Zingiberaceae } & Curcuma Zedoaria & no & also C. phaeocaulis; several synonyms \\
\hline & Hedychium Gardnerianum & yes & H. gardnerianum \\
\hline & Brachychilum sp. & yes & some taxa accepted, some in Hedychium \\
\hline & Kämpferia sp. & no & Kaempferia \\
\hline & Alpinia nutans & yes & $\begin{array}{l}\text { accepted names } A . \text { zerumbet and } A . \\
\text { speciosa; several synonyms }\end{array}$ \\
\hline & Alpinia vittata & no & \\
\hline & Zingiber Zerumbet & no & $\begin{array}{l}\text { it is not clear if such species exists: it } \\
\text { could be Alpinia zerumbe }\end{array}$ \\
\hline & Amomum Melegueta & no & $\begin{array}{l}\text { accepted } A \text {. melegueta, also Aframomum } \\
\text { melegueta }\end{array}$ \\
\hline & Globba spp. & no & \\
\hline & $\begin{array}{l}\text { "and many others of the same } \\
\text { family" }\end{array}$ & yes & \\
\hline
\end{tabular}


Table 2. Collection of the temperate (warm) glasshouse between 1948 and 2014: recently non-existing plants

\begin{tabular}{|c|c|c|c|c|c|}
\hline 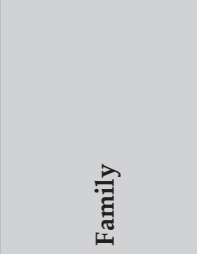 & 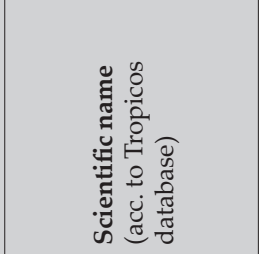 & 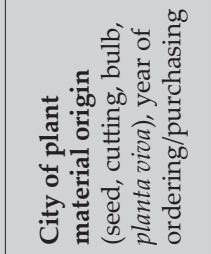 & 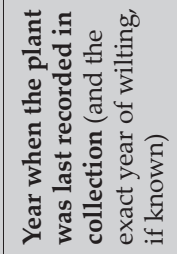 & 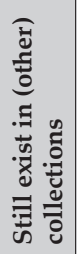 & 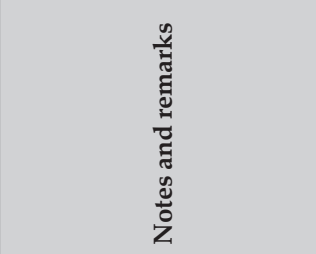 \\
\hline & $\begin{array}{l}\text { THALLOSE } \\
\text { LIVERWORTS }\end{array}$ & & & & \\
\hline \multirow[t]{3}{*}{ Aytoniaceae } & $\begin{array}{l}\text { Fimbriaria ludwigii } \\
\text { (Schwaegr.) Limpr. } \\
\text { ex Muell. Frib. }\end{array}$ & Jena, 1963 \& 1964 & $1963 \& 1966$ & no & syn. Marchantia ludwigii \\
\hline & & $\begin{array}{l}\text { Antwerp, } 1967 \& \\
1968\end{array}$ & 1968 & no & \\
\hline & $\begin{array}{l}\text { Mannia dichotoma } \\
\text { S.W. Arnell }\end{array}$ & Jena, 1966 & 1968 & no & older: Grimaldia d. \\
\hline Corsiniaceae & $\begin{array}{l}\text { Corsinia marchantio- } \\
\text { ides Raddi }\end{array}$ & Antwerp, 1968 & 1968 & no & \\
\hline \multirow[t]{3}{*}{ Lunulariaceae } & $\begin{array}{l}\text { Lunularia cruciata (L.) } \\
\text { Dumort. ex Lindb. }\end{array}$ & nn, 1949 & 1961 & yes & \\
\hline & & Jena, 1963 & 1963 & yes & \\
\hline & & Antwerp, 1965 & 1966 & yes & \\
\hline \multirow[t]{8}{*}{ Marchantiaceae } & $\begin{array}{l}\text { Dumortiera hirsuta } \\
\text { (Sw.) Nees }\end{array}$ & Jena, 1963 & 1963 & no & \\
\hline & & Budapest, 1963 & 1963 & no & \\
\hline & & Antwerp, 1966 & 1968 & no & \\
\hline & $\begin{array}{l}\text { Dumortiera velutina } \\
\text { Schiffn. }\end{array}$ & Jena, 1964 & 1966 & no & $\begin{array}{l}\text { not listed in Tropicos, Plant } \\
\text { List nor IPNI; Catalogue of } \\
\text { Life states that it is one of } \\
\text { the many synonyms of } D \text {. } \\
\text { hirsuta }\end{array}$ \\
\hline & & $\begin{array}{l}\text { Antwerp, 1965, } \\
\text { 1966 \& } 1967\end{array}$ & $\begin{array}{l}1966,1968 \& \\
1969\end{array}$ & no & \\
\hline & $\begin{array}{l}\text { Marchantia paleacea } \\
\text { Bertol. }\end{array}$ & $\begin{array}{l}\text { Antwerp, 1962, } \\
1964 \text { \& } 1965\end{array}$ & $\begin{array}{l}1963,1966 \& \\
1966\end{array}$ & no & \\
\hline & & Jena, 1963 & 1966 & no & \\
\hline & $\begin{array}{l}\text { Marchantia } \\
\text { polymorpha } \mathrm{L} .\end{array}$ & Jena, $1970 \& 1981$ & $\begin{array}{l}\text { no furter } \\
\text { records }\end{array}$ & yes & replaced? \\
\hline \multirow[t]{3}{*}{ Targioniaceae } & $\begin{array}{l}\text { Targionia hypophylla } \\
\text { L. }\end{array}$ & Jena, 1963 \& 1966 & $\begin{array}{l}\text { no further } \\
\text { record \& } 1968\end{array}$ & no & \\
\hline & & Antwerp, 1968 & $\begin{array}{l}\text { no further } \\
\text { record }\end{array}$ & no & \\
\hline & $\begin{array}{l}\text { Targionia lorbeeriana } \\
\text { Muell. Frib. }\end{array}$ & $\begin{array}{l}\text { Antwerp, 1965, } \\
\text { 1966 \& } 1968\end{array}$ & $\begin{array}{l}1966,1968 \& \\
1968\end{array}$ & no & \\
\hline \multirow[t]{2}{*}{ Wiesnerellaceae } & $\begin{array}{l}\text { Wiesnerella denudata } \\
\text { (Mitt.) Stephani }\end{array}$ & Jena, 1966 & 1968 & no & \\
\hline & SPIKEMOSSES & & & & \\
\hline Selaginellaceae & $\begin{array}{l}\text { Selaginella apoda (L.) } \\
\text { C. Morren }\end{array}$ & nn, 1961 & 1984 & no & \\
\hline
\end{tabular}




\begin{tabular}{|c|c|c|c|c|c|}
\hline & $\begin{array}{l}\text { Selaginella biformis A. } \\
\text { Braun ex Kuhn }\end{array}$ & Lyon, 1965 & $1968(1975)$ & no & \\
\hline & $\begin{array}{l}\text { Selaginella delicatula } \\
\text { (Desv. ex Poir.) } \\
\text { Alston }\end{array}$ & Lyon, 1965 & $\begin{array}{l}\text { no further } \\
\text { record }\end{array}$ & no & $\begin{array}{l}\text { arrived as S. canaliculata, } \\
\text { one of about } 10 \text { recognized } \\
\text { synonyms }\end{array}$ \\
\hline & $\begin{array}{l}\text { Selaginella erythropus } \\
\text { (Mart.) Spring }\end{array}$ & Oslo, 1957 & 1966 (1975) & no & $\begin{array}{l}\text { arrived with the synonym } \\
\text { "S. umbrosa", which is } \\
\text { nowdays syn. of } S \text {. } \\
\text { erythropus var. major }\end{array}$ \\
\hline & $\begin{array}{l}\text { Selaginella haematodes } \\
\text { (Kunze) Spring }\end{array}$ & Szeged, 1963 & 1975 & no & \\
\hline & & Lyon, 1963 & 1975 & no & \\
\hline & $\begin{array}{l}\text { Selaginella involvens } \\
\text { (Sw.) Spring }\end{array}$ & nn, 1950 & 1952 & no & $\begin{array}{l}\text { arrived as } S . \text { caulescens; } \\
\text { there are atleast } 3 \text { more } \\
\text { accepted names and many } \\
\text { synonyms }\end{array}$ \\
\hline & $\begin{array}{l}\text { Selaginella kraussiana } \\
\text { (Kunze) A. Braun }\end{array}$ & nn, 1961 & $\begin{array}{l}\text { no further } \\
\text { record }\end{array}$ & no & $\begin{array}{l}\text { synonyms are also } S \text {. } \\
\text { cansecens and } S \text {. denticulata }\end{array}$ \\
\hline & & Besancon, 1963 & $\begin{array}{l}\text { no further } \\
\text { record }\end{array}$ & no & \\
\hline & & Szeged, 1963 & $\begin{array}{l}\text { no further } \\
\text { record }\end{array}$ & no & \\
\hline & & Genova, 1963 & $\begin{array}{l}\text { no further } \\
\text { record }\end{array}$ & no & \\
\hline & & Cork, 1963 & $\begin{array}{l}1988 \\
\text { ("incorrect!") }\end{array}$ & no & \\
\hline & & Antwerp, 1968 & 1969 & no & arrived as S. denticulata \\
\hline & $\begin{array}{l}\text { Selaginella kraussiana } \\
\text { (Kunze) A. Braun } \\
\text { 'Aurea' }\end{array}$ & Lyon, 1965 & 1965 (1975) & no & arrived as "var. aurea" \\
\hline & $\begin{array}{l}\text { Selaginella lepidophylla } \\
\text { (Hook. \& Grev.) } \\
\text { Spring }\end{array}$ & nn, 2007 & 2009 & no & $\begin{array}{l}\text { given to the students' } \\
\text { laboratory }\end{array}$ \\
\hline & \begin{tabular}{|l|} 
Selaginella lyallii \\
(Hook. \& Grev.) \\
Spring
\end{tabular} & $\begin{array}{l}\text { Lyon, } 1963 \& \\
1965\end{array}$ & $\begin{array}{l}\text { no further } \\
\text { records (both) }\end{array}$ & no & $\begin{array}{l}\text { arrived as S. laevigata var. } \\
\text { lyallii }\end{array}$ \\
\hline & $\begin{array}{l}\text { Selaginella martensii } \\
\text { Spring var. } \\
\text { watsoniana (?) }\end{array}$ & Antwep, 1968 & 1969 (1975) & no & $\begin{array}{l}\text { arrived as "var. aurea"; the } \\
\text { variety is unknown to } \\
\text { Tropicos, it could be } \\
\text { "hort." }\end{array}$ \\
\hline & $\begin{array}{l}\text { Selaginella } \\
\text { millspaughii Hieron. }\end{array}$ & Antwerp, 1962 & 1966 & no & 3 other accepted names \\
\hline & & \begin{tabular}{|l|} 
Lyon, 1963, 1964, \\
1965 \& 1966
\end{tabular} & $\begin{array}{l}1975,1968, \\
1978 \& 1978 \\
\end{array}$ & no & \\
\hline & & Besancon, 1965 & 1966 & no & \\
\hline & $\begin{array}{l}\text { Selaginella pilifera A. } \\
\text { Braun }\end{array}$ & Lyon, 1965 & 1969 (1975) & no & \\
\hline & $\begin{array}{l}\text { Selaginella plana } \\
\text { (Desv. ex Poir.) } \\
\text { Hieron. }\end{array}$ & Lyon, 1963 & $\begin{array}{l}\text { no further } \\
\text { record }\end{array}$ & no & \\
\hline & & Cork, 1963 & & no & \\
\hline & $\begin{array}{l}\text { Selaginella stellata } \\
\text { Spring }\end{array}$ & Antwerp, 1962 & $\begin{array}{l}1988 \\
\text { ("incorrect!") }\end{array}$ & no & \\
\hline & & $\begin{array}{l}\text { Lyon, } 1963 \& \text { \& } \\
1965\end{array}$ & $\begin{array}{l}1966 \& \text { no } \\
\text { further record }\end{array}$ & no & $\begin{array}{l}\text { arrived as } S . \text { galeottii } \\
\text { Spring, a single synonym }\end{array}$ \\
\hline
\end{tabular}




\begin{tabular}{|c|c|c|c|c|c|}
\hline & & Szeged, 1965 & 1966 & no & 1 synonym \\
\hline & & Besancon, 1965 & 1988 & no & $\begin{array}{l}\text { arrived as } S . \text { galeottii } \\
\text { Spring, a single synonym }\end{array}$ \\
\hline & $\begin{array}{l}\text { Selaginella uncinata } \\
\text { (Desv. ex Poir.) } \\
\text { Spring } \\
\end{array}$ & nn, 1951 & 1966 & no & 1 synonym \\
\hline & & Lyon, 1966 & $1966(1975)$ & no & \\
\hline & $\begin{array}{l}\text { Selaginella usteri } \\
\text { Hieron. }\end{array}$ & Pisa, 1963 & $1969(1975)$ & no & \\
\hline & $\begin{array}{l}\text { Selaginella victoriae } \\
\text { Moor. }\end{array}$ & Antwerp, 1968 & $1969(1975)$ & no & $\begin{array}{l}\text { not recognized in Tropicos } \\
\text { nor IPNI; acc. to the } \\
\text { caverock.net.nz' World } \\
\text { Species list of Selaginel- } \\
\text { laceae, it is a forgotten } \\
\text { synonym of S. atroviridis } \\
\text { Spring }\end{array}$ \\
\hline & $\begin{array}{l}\text { Selaginella viticulosa } \\
\text { Klotzsch }\end{array}$ & nn, 1952 & $1969(1975)$ & no & \\
\hline & $\begin{array}{l}\text { Selaginella watsonii } \\
\text { Underw. }\end{array}$ & $\begin{array}{l}\text { Antwerp, } 1964 \& \\
1965\end{array}$ & $\begin{array}{l}\text { no further } \\
\text { record \& } 1966\end{array}$ & no & \\
\hline & $\begin{array}{l}\text { Selaginella willdenovii } \\
\text { Baker }\end{array}$ & Oslo, 1965 & $\begin{array}{l}\text { no further } \\
\text { record (1975) }\end{array}$ & no & \\
\hline & FERNS & & & & \\
\hline Anemiaceae & $\begin{array}{l}\text { Anemia rotundifolia } \\
\text { Schrad. }\end{array}$ & Marburg, 1982 & 1982 & no & \\
\hline & & Karlsruhe, 1982 & 1988 & no & \\
\hline Aspleniaceae & $\begin{array}{l}\text { Asplenium bulbiferum } \\
\text { G. Forst. }\end{array}$ & Haren, 1974 & 1975 & no & 4 synonyms \\
\hline & & Strasbourg, 1986 & 1988 & no & \\
\hline & $\begin{array}{l}\text { Asplenium nidus L. } \\
\text { 'Osaka' }\end{array}$ & nn, 2008 & 2009 & no & $\begin{array}{l}\text { purchased; famous old } \\
\text { cultivar }\end{array}$ \\
\hline & $\begin{array}{l}\text { Asplenium } \\
\text { scolopendrium } \mathrm{L} . \\
\text { Crispum Group }\end{array}$ & Essen, 2001 & 2005 (2006) & no & $\begin{array}{l}\text { arrived as Phyllitis 'Crispa'; } \\
\text { several synonyms \& } \\
\text { accepted names of species }\end{array}$ \\
\hline Blechnaceae & $\begin{array}{l}\text { Blechnum brasiliense } \\
\text { Desv. }\end{array}$ & nn, 1953 & $\begin{array}{l}\text { no further } \\
\text { record }\end{array}$ & no & 6 synonyms \\
\hline & & $\begin{array}{l}\text { Amsterdam, } \\
1968\end{array}$ & 1969 & no & \\
\hline & & Berlin, 1969 & 1971 & no & \\
\hline & $\begin{array}{l}\text { Blechnum moorei } \\
\text { C.Chr. }\end{array}$ & Kiel, 1982 & 1988 & no & $\begin{array}{l}\text { acc. to IPNI \& Plant List; an } \\
\text { unresolved name }\end{array}$ \\
\hline & & Muenchen, 1982 & 2000 & no & \\
\hline & $\begin{array}{l}\text { Blechnum neohollandi- } \\
\text { cum Christenh. }\end{array}$ & Lyon, 1954 & 1963 & no & $\begin{array}{l}\text { arrived as syn. Doodia } \\
\text { aspera, which is replaced by } \\
\text { B. } n \text {. }\end{array}$ \\
\hline & $\begin{array}{l}\text { Stenochlaena palustris } \\
\text { (Burm. f.) Bedd. }\end{array}$ & Antwerp, 1966 & 1968 & no & 10+ synonyms \\
\hline & $\begin{array}{l}\text { Stenochlaena tenuifolia } \\
\text { (Desv.) T. Moore }\end{array}$ & Besancon, 1977 & 1980 & no & $10+$ synonyms \\
\hline Cyatheaceae & $\begin{array}{l}\text { Cyathea australis } \\
\text { Domin }\end{array}$ & $\begin{array}{l}\text { Amsterdam, } \\
1968 \\
\end{array}$ & 1969 & no & $\begin{array}{l}\text { arrived as syn. Alsophila } \\
\text { australis }\end{array}$ \\
\hline & & Potsdam, 2010 & 2012 (2013) & no & \\
\hline
\end{tabular}




\begin{tabular}{|c|c|c|c|c|c|}
\hline & $\begin{array}{l}\text { Cyathea leichhardtiana } \\
\text { Copel. }\end{array}$ & $\begin{array}{l}\text { Amsterdam, } \\
1968 \\
\end{array}$ & 1969 & no & $\begin{array}{l}\text { arrived as syn. } A . \\
\text { leichardtiana }\end{array}$ \\
\hline \multirow[t]{2}{*}{ Cystopteridaceae } & $\begin{array}{l}\text { Cystopteris bulbifera } \\
\text { (L.) Bernh. }\end{array}$ & Bologna, 1974 & 1975 & no & 6 synonyms \\
\hline & & Berlin, 1989 & 1996 & no & \\
\hline \multirow[t]{8}{*}{ Davalliaceae } & $\begin{array}{l}\text { Davallia denticulata } \\
\text { (Burm. f.) Mett. ex } \\
\text { Kuhn }\end{array}$ & $\begin{array}{l}\text { Antwerp, } 1967 \& \\
1973\end{array}$ & $1968 \& 1978$ & no & $\begin{array}{l}\text { accepted name is also } D . \\
\text { chaerophylloides }\end{array}$ \\
\hline & $\begin{array}{l}\text { Davallia solida (G. } \\
\text { Forst.) Sw. }\end{array}$ & Tuebingen, 1971 & 1972 & yes & $\begin{array}{l}\text { accepted name is also } D \text {. } \\
\text { sinensis }\end{array}$ \\
\hline & $\begin{array}{l}\text { Nephrolepis biserrata } \\
\text { (Sw.) Schott var. } \\
\text { furcans L.H. Bailey }\end{array}$ & Montreal, 1978 & 1979 & no & also as 'Furcans' hort. \\
\hline & $\begin{array}{l}\text { Nephrolepis cordifolia } \\
\text { (L.) C. Presl }\end{array}$ & Szeged, 1970 & $1980(1988)$ & no & 12 synonyms \\
\hline & $\begin{array}{l}\text { Nephrolepis exaltata } \\
\text { (L.) Schott f. muscosa } \\
\text { Clute }^{*}\end{array}$ & nn, 1963 & $\begin{array}{l}1969 \\
\text { ("incorrect!") }\end{array}$ & no & $\begin{array}{l}\text { f. acc. to IPNI (as fam. } \\
\text { Oleandraceae) }\end{array}$ \\
\hline & $\begin{array}{l}\text { Nephrolepis exaltata } \\
\text { (L.) Schott 'Green } \\
\text { Fantasy' }\end{array}$ & nn, 2003 & 2005 (2006) & no & $\begin{array}{l}\text { purchased; well known } \\
\text { cultivar }\end{array}$ \\
\hline & $\begin{array}{l}\text { Nephrolepis exaltata } \\
\text { (L.) Schott 'Whitma- } \\
\text { nii' }\end{array}$ & Opeka, 1963 & 1978 & no & 4 species-synonyms \\
\hline & $\begin{array}{l}\text { Nephrolepis hirsutula } \\
\text { (G. Forst.) C. Presl }\end{array}$ & Szeged, 2002 & 2007 (2008) & no & 3 synonyms \\
\hline \multirow[t]{2}{*}{ Dennstaedtiaceae } & $\begin{array}{l}\text { Histiopteris incisa } \\
\text { (Thunb.) J. Sm. }\end{array}$ & $\begin{array}{l}\text { Leningrad (St. } \\
\text { Petersburg), } 1981\end{array}$ & 1988 & no & $\begin{array}{l}\text { arrived as syn. Pteris } \\
\text { quadriaurita (Pteridaceae); } 9 \\
\text { synonyms; perhaps the } \\
\text { family is monotypic }\end{array}$ \\
\hline & $\begin{array}{l}\text { Microlepia speluncae } \\
\text { (L.) T. Moore }\end{array}$ & Muenchen, 1958 & 1961 & no & $\begin{array}{l}\text { accepted name is also } M . \\
\text { hancei; } 30+\text { synonyms }\end{array}$ \\
\hline Dicksoniaceae & $\begin{array}{l}\text { Dicksonia antarctica } \\
\text { Labill. }\end{array}$ & Potsdam, 2010 & 2012 & no & \\
\hline \multirow[t]{8}{*}{ Dryopteridaceae } & $\begin{array}{l}\text { Arachniodes aristata } \\
\text { (G. Forst.) Tindale }\end{array}$ & Berlin, 1968 & 1972 (1988) & no & $\begin{array}{l}\text { arrived as syn. Polystichum } \\
\text { aristatum; 20+ synonyms }\end{array}$ \\
\hline & $\begin{array}{l}\text { Cyrtomium falcatum } \\
\text { (L. f.) C. Presl } \\
\text { 'Rochfordianum' }\end{array}$ & Stockholm, 1958 & 1988 & no & 7 species-synonyms \\
\hline & & $\begin{array}{l}\text { Cluj-Napoca, } \\
1969 \\
\end{array}$ & 1975 & no & \\
\hline & $\begin{array}{l}\text { Cyrtomium } \\
\text { lonchitoides (Christ) } \\
\text { Christ } \\
\end{array}$ & nn, 1961 \& 1963 & $\begin{array}{l}1988 \& 2001 \\
(2003)\end{array}$ & no & $\begin{array}{l}\text { arrived as Polystichum } \\
\text { lonchitoides, one of } 4 \text { today's } \\
\text { synonyms }\end{array}$ \\
\hline & $\begin{array}{l}\text { Diacalpe aspidioides } \\
\text { Blume }\end{array}$ & nn, 1961 & 2000 & no & $\begin{array}{l}2 \text { more accepted names } \\
\text { (Peranema a., Dryopteris } \\
\text { pseudocaenopteris) }\end{array}$ \\
\hline & & nn, 2005 & 2005 (2007) & no & purchased \\
\hline & \begin{tabular}{|l|}
$\begin{array}{l}\text { Polystichum braunii } \\
\text { (Spenn.) Fée }\end{array}$ \\
\end{tabular} & nn, 1961 & $\begin{array}{l}\text { no further } \\
\text { record }\end{array}$ & no & 4 species-synonyms \\
\hline & \begin{tabular}{|l|} 
Polystichum \\
polyblepharum (Roem. \\
ex Kunze) C. Presl \\
\end{tabular} & nn, 2007 & 2008 & no & 3 synonyms \\
\hline Lygodiaceae & $\begin{array}{l}\text { Lygodium japonicum } \\
\text { (Thunb.) Sw. }\end{array}$ & Lyon, 1966 & $\begin{array}{l}\text { no further } \\
\text { record }\end{array}$ & no & $15+$ synonyms \\
\hline
\end{tabular}




\begin{tabular}{|c|c|c|c|c|c|}
\hline & & Brno, 2001 & $\begin{array}{l}2003 \\
\text { (removed) }\end{array}$ & no & \\
\hline & & Lyon, 1967 & $\begin{array}{l}\text { no further } \\
\text { record }\end{array}$ & no & \\
\hline Onocleaceae & Onoclea sensibilis L. & $\begin{array}{l}\text { Amsterdam, } \\
1958\end{array}$ & 1963 & no & $\begin{array}{l}8 \text { synonyms; perhaps the } \\
\text { family is monotypic }\end{array}$ \\
\hline \multirow[t]{8}{*}{ Polypodiaceae } & $\begin{array}{l}\text { Drynaria quercifolia } \\
\text { (L.) J. Sm. }\end{array}$ & Aachen, 1990 & 1991 & no & syn. Polypodium q. \\
\hline & & Berlin, 1969 & $\begin{array}{l}1975 \\
\text { ("incorrect!") }\end{array}$ & no & \\
\hline & $\begin{array}{l}\text { Microsorum } \\
\text { punctatum (L.) Copel. }\end{array}$ & nn, $1961 \& 1969$ & 2000 & no & $\begin{array}{l}\text { arrived as M. polycarpon, } \\
\text { which is also an accepted } \\
\text { name; contains } 60+ \\
\text { synonyms }\end{array}$ \\
\hline & & $\begin{array}{l}\text { nn, 1961, 1972, } \\
1981\end{array}$ & $\begin{array}{l}1981 \\
(\text { "incorrect!"), } \\
1991\end{array}$ & no & $\begin{array}{l}\text { arrived as Microsorium } p . ; \\
\text { accepted name is also } M . \\
\text { polycarpon; contains } 60+ \\
\text { synonyms }\end{array}$ \\
\hline & $\begin{array}{l}\text { Platycerium } \\
\text { ‘Wilhelminae-Regi- } \\
\text { nae' }\end{array}$ & Potsdam, 1982 & 1982 & no & $\begin{array}{l}\text { ?, probably Platycerium } \\
\text { grande J. Sm. 'Queen } \\
\text { Wilhelmina' }\end{array}$ \\
\hline & $\begin{array}{l}\text { Polypodium glaucum } \\
\text { Thunb. ex Houtt. }\end{array}$ & $\begin{array}{l}\text { Cluj-Napoca, } \\
1968\end{array}$ & 1973 & no & $\begin{array}{l}\text { with } 4 \text { accepted names; } \\
\text { several synonyms }\end{array}$ \\
\hline & $\begin{array}{l}\text { Polypodium leiorhizum } \\
\text { Wall.* }\end{array}$ & Karlsruhe, 1969 & $\begin{array}{l}1973 \\
\text { ("incorrect: } \\
\text { Pteris!") }\end{array}$ & no & $\begin{array}{l}\text { that name is marked as } \\
\text { "unsolved" in all consulted } \\
\text { databases }\end{array}$ \\
\hline & $\begin{array}{l}\text { Pyrrosia } \\
\text { nummulariifolia (Sw.) } \\
\text { Ching }\end{array}$ & Leiden, 1985 & 2007 (2008) & no & $10+$ synonyms \\
\hline \multirow[t]{11}{*}{ Pteridaceae } & $\begin{array}{l}\text { Adiantum } \\
\text { ‘Magnificum' (?) }\end{array}$ & \begin{tabular}{|l|} 
Bucarest, $1986 \&$ \& \\
1988
\end{tabular} & $1988 \& 1989$ & no & unknown cultivar \\
\hline & $\begin{array}{l}\text { Adiantum raddianum } \\
\text { C. Presl 'Gracilli- } \\
\text { mum' }\end{array}$ & Riga, 1959 & $2010(2011)$ & no & $\begin{array}{l}\text { arrived under also } \\
\text { accepted name: } A \text {. } \\
\text { cuneatum var. gracillimum }\end{array}$ \\
\hline & $\begin{array}{l}\text { Adiantum raddianum } \\
\text { 'Brilliantelse' or } \\
\text { 'Brilliant Elze' }\end{array}$ & Opeka, 1963 & 1968 & no & arrived as $A$. brillantelse \\
\hline & $\begin{array}{l}\text { Adiantum tenerum } \\
\text { Sw. (cult.?) }\end{array}$ & Opeka, 1963 & 1969 & no & $\begin{array}{l}\text { arrived as syn. A. tenerum } \\
\text { var. scutatum hort f. roseum, } \\
\text { hard to tell which cultivar } \\
\text { it was, perhaps 'Scutum } \\
\text { Roseum' }\end{array}$ \\
\hline & $\begin{array}{l}\text { Adiantum } \\
\text { tetraphyllum Humb. } \\
\text { \& Bonpl. ex Willd.* }\end{array}$ & Szeged, 1970 & \begin{tabular}{|l|}
1984 \\
("incorrect: \\
A.capillus- \\
veneris!") \\
\end{tabular} & no & 4 synonyms \\
\hline & $\begin{array}{l}\text { Pellaea atropurpurea } \\
\text { (L.) Link }\end{array}$ & Muenchen, 1997 & 2005 (2006) & no & $\begin{array}{l}5 \text { synonyms; there is also } P \text {. } \\
\times \text { atropurpurea (pro. sp.) }\end{array}$ \\
\hline & $\begin{array}{l}\text { Pellaea paradoxa } \\
\text { Hook. "Glow Star" }\end{array}$ & nn, 2007 & 2008 & no & $\begin{array}{l}\text { purchased; well known } \\
\text { cultivar }\end{array}$ \\
\hline & $\begin{array}{l}\text { Pellaea rotundifolia (G. } \\
\text { Forst.) Hook. }\end{array}$ & Cork, 1960 & 1961 & no & \\
\hline & & Zurich, 1982 & 1983 & no & \\
\hline & & Marburg, 1982 & 2007 (2008) & no & \\
\hline & $\begin{array}{l}\text { Pityrogramma } \\
\text { calomelanos (L.) Link }\end{array}$ & Berlin, 1968 & 1969 & no & $15+$ synonyms \\
\hline
\end{tabular}




\begin{tabular}{|c|c|c|c|c|c|}
\hline & $\begin{array}{l}\text { Pityrogramma } \\
\text { sulphurea (Sw.) } \\
\text { Maxon }\end{array}$ & nn, 1954 \& 1982 & $\begin{array}{l}\text { no further } \\
\text { record \& } 1984\end{array}$ & no & \\
\hline & & Muenster, 1988 & 1990 & no & \\
\hline & & Dresden, 1989 & 1990 & no & \\
\hline & & nn, 2005 & 2005 & no & purchased \\
\hline & $\begin{array}{l}\text { Pteris argyraea } \mathrm{T} . \\
\text { Moore }\end{array}$ & Opeka, 1963 & 1972 & no & \\
\hline & & $\begin{array}{l}\text { Nancy, } 1968 \& \\
1982\end{array}$ & $1975 \& 1991$ & no & \\
\hline & & Potsdam, 1982 & 1991 & no & \\
\hline & & Muenchen, 1982 & 1991 & no & \\
\hline & Pteris cretica L. & nn, 1961 & 2010 & no & $\begin{array}{l}\text { accepted name is also } P . \\
\text { stenophylla; } 10 \text { synonyms }\end{array}$ \\
\hline & $\begin{array}{l}\text { Pteris cretica L. } \\
\text { 'Gauthierii' (?) }\end{array}$ & Opeka, 1963 & 1978 & no & $\begin{array}{l}\text { accepted species-name is } \\
\text { also } P \text {. stenophylla; } 10 \\
\text { species-synonyms; the } \\
\text { cultivar is unknown, } \\
\text { maybe 'Gautheri'? }\end{array}$ \\
\hline & $\begin{array}{l}\text { Pteris cretica L. } \\
\text { 'Rowerii' }\end{array}$ & Opeka, 1963 & 1966 & no & $\begin{array}{l}\text { accepted name is also } P \text {. } \\
\text { stenophylla; } 10 \text { species- } \\
\text { synonyms; the cultivar } \\
\text { arrived as 'Roeweri' }\end{array}$ \\
\hline & $\begin{array}{l}\text { Pteris multifida Poir. } \\
\text { 'Cristata' }\end{array}$ & nn, 1975 & 1978 & no & 3 species-synonyms \\
\hline & Pteris vittata $\mathrm{L}$. & nn, $1961 \& 1963$ & 1981 & no & $\begin{array}{l}\text { arrived as } P \text {. longifolia, } \\
\text { accepted name of } P \text {. vittata } \\
\text { together with } 2 \text { others; } 10 \\
\text { synonyms }\end{array}$ \\
\hline & & Utrecht, 1980 & 2010 & no & 10 synonyms \\
\hline & $\begin{array}{l}\text { DICOTYLEDONS } \\
\text { (Magnoliopsida) }\end{array}$ & & & & \\
\hline \multirow[t]{11}{*}{ Acanthaceae } & $\begin{array}{l}\text { Aphelandra aurantiaca } \\
\text { (Scheidw.) Lindl. }\end{array}$ & $\begin{array}{l}\text { Rouen, } 1961 \& \\
1966\end{array}$ & $1962 \& 1968$ & no & 10 synonyms \\
\hline & $\begin{array}{l}\text { Aphelandra squarrosa } \\
\text { Nees }\end{array}$ & nn, 1961 & $\begin{array}{l}\text { no further } \\
\text { record }\end{array}$ & no & \\
\hline & $\begin{array}{l}\text { Barleria lupulina } \\
\text { Lindl. }\end{array}$ & Bogor, 2004 & 2005 & no & 2 synonyms \\
\hline & Barleria prionitis $\mathrm{L}$. & Wuppertal, 1964 & $\begin{array}{l}\text { no further } \\
\text { record }\end{array}$ & no & 5 synonyms \\
\hline & & Glasgow, 1987 & 1988 & no & \\
\hline & & $\begin{array}{l}\text { Copenhagen, } \\
1986\end{array}$ & 1988 & no & \\
\hline & $\begin{array}{l}\text { Brillantaisia lamium } \\
\text { (Nees) Benth. }\end{array}$ & $\begin{array}{l}\text { Saarbruecken, } \\
1968\end{array}$ & 1969 & no & syn. Leucorhaphis lamium \\
\hline & $\begin{array}{l}\text { Brillantaisia palisotii } \\
\text { Lindau }\end{array}$ & Goettingen, 1965 & 1988 & no & \\
\hline & $\begin{array}{l}\text { Chamaeranthemum } \\
\text { beyrichii Nees }\end{array}$ & Aachen, 1966 & 1977 & no & \\
\hline & $\begin{array}{l}\text { Crabbea velutina S. } \\
\text { Moore }\end{array}$ & Besancon, 1965 & 1969 & no & $\begin{array}{l}\text { arrived as C. reticulata, one } \\
\text { of } 2 \text { synonyms }\end{array}$ \\
\hline & & Jena, 1964 & 1988 & no & \\
\hline
\end{tabular}


S14 Kovačić, S.: Plethora of plants - collections of the Botanical Garden, Faculty of Science, University of Zagreb (1)

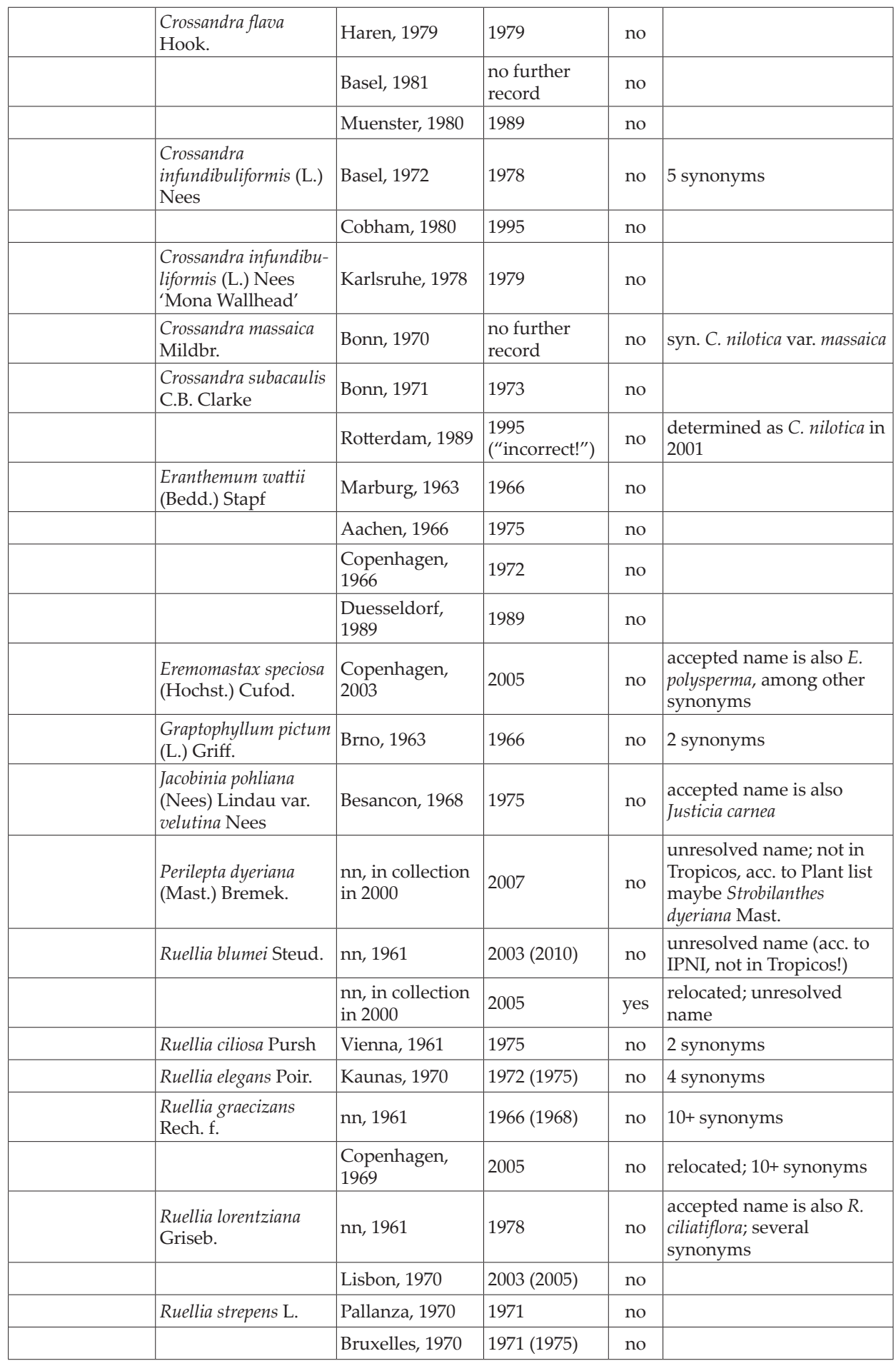




\begin{tabular}{|c|c|c|c|c|c|}
\hline & Ruellia tuberosa $\mathrm{L}$. & nn, 1961 & 2003 & no & \\
\hline & $\begin{array}{l}\text { Ruspolia seticalyx } \\
\text { (C.B. Clarke) } \\
\text { Milne-Redh. }\end{array}$ & Berlin, 1967 & $1984(1988)$ & no & \\
\hline & & Turlen, 1989 & 2001 & no & \\
\hline & $\begin{array}{l}\text { Strobilanthes glabrata } \\
\text { Nees }\end{array}$ & Tuebingen, 1963 & 1966 (1975) & no & \\
\hline & $\begin{array}{l}\text { Strobilanthes "roseus } \\
\text { Nees" (?) }\end{array}$ & Kaunas 1962 & $1968(1975)$ & no & $\begin{array}{l}\text { unresolved name (acc. to } \\
\text { IPNI, not in Tropicos nor } \\
\text { Plant List); perhaps Ruellia } \\
\text { rosea }\end{array}$ \\
\hline & $\begin{array}{l}\text { Thunbergia fragrans } \\
\text { Roxb. }\end{array}$ & Bonn, 1987 & 1991 & no & $\begin{array}{l}\text { accepted name is also Th. } \\
\text { convolvulifolia; } 6 \text { synonyms }\end{array}$ \\
\hline & $\begin{array}{l}\text { Thunbergia grandiflora } \\
\text { Roxb. }\end{array}$ & Barcelona, 1963 & 1972 & no & 5 synonyms \\
\hline & $\begin{array}{l}\text { Whitfieldia longifolia } \\
\text { T. Anderson }\end{array}$ & Caen, 2002 & $2003(2010)$ & no & $\begin{array}{l}\text { accepted name is also } \\
\text { Whitfieldia elongata, syn. } \\
\text { Ruellia elongata and } 4 \text { more }\end{array}$ \\
\hline Amaranthaceae & Iresine herbstii Hook. & nn, 1981 & 2005 & yes & $\begin{array}{l}3 \text { accepted names, } 2 \\
\text { synonyms }\end{array}$ \\
\hline \multirow[t]{2}{*}{ Anacardiaceae } & Mangifera indica $\mathrm{L}$. & nn, 1981 & 1984 & no & 2 synonyms \\
\hline & $\begin{array}{l}\text { Maytenus "serrotina" } \\
(?)\end{array}$ & Muenchen, 1979 & 1988 & no & $\begin{array}{l}\text { unknown species, perhaps } \\
\text { a misspelling; maybe "M. } \\
\text { serrata"...? }\end{array}$ \\
\hline Annonaceae & Annona reticulata L. & Potsdam, 1982 & 1984 & no & $\begin{array}{l}\text { accepted names are also } A \text {. } \\
\text { muscosa and Rollinia } \\
\text { mucosa; } 20+\text { synonyms }\end{array}$ \\
\hline \multirow[t]{13}{*}{ Apocynaceae } & $\begin{array}{l}\text { Acokanthera spectabilis } \\
\text { (Sond.) Hook. f. }\end{array}$ & Dublin, 1964 & 1965 & no & $\begin{array}{l}\text { accepted name is also } \\
\text { A.oblongifolia; } 2 \text { synonyms }\end{array}$ \\
\hline & $\begin{array}{l}\text { Carissa macrocarpa } \\
\text { (Eckl.) A. DC. }\end{array}$ & Antibes, 1978 & 1981 & no & $\begin{array}{l}\text { arrived as C. grandiflora, } \\
\text { one of } 4 \text { synonyms }\end{array}$ \\
\hline & $\begin{array}{l}\text { Cryptolepis } \\
\text { triangularis N.E. Br. }\end{array}$ & “Angola", 1972 & 1975 & no & $\begin{array}{l}\text { possibly from the native } \\
\text { (Angolan) locality, via } \\
\text { some Delectus Seminum }\end{array}$ \\
\hline & $\begin{array}{l}\text { Mandevilla suaveolens } \\
\text { Lindl. }\end{array}$ & Coimbra, 1953 & $\begin{array}{l}\text { no further } \\
\text { record }\end{array}$ & no & $\begin{array}{l}\text { accepted name is also } M . \\
\text { laxa; } 10 \text { synonyms }\end{array}$ \\
\hline & & Lisbon, 1960 & 1968 & no & \\
\hline & $\begin{array}{l}\text { Philibertia gracilis D. } \\
\text { Don }\end{array}$ & $\begin{array}{l}\text { Besancon, } 1962 \\
\& 1965\end{array}$ & \begin{tabular}{|l|}
$1963 \&$ no \\
further record
\end{tabular} & no & 3 more accepted names \\
\hline & $\begin{array}{l}\text { Rauvolfia tetraphylla } \\
\text { L. }\end{array}$ & Muenchen, 1980 & 1988 & no & $15+$ synonyms \\
\hline & & Shanghai, 1980 & 1988 & no & $\begin{array}{l}\text { arrived as Rauwolfia t.; one } \\
\text { of } 15+\text { synonyms }\end{array}$ \\
\hline & & $\begin{array}{l}\text { Marburg, } 1958 \& \\
1959\end{array}$ & $\begin{array}{l}1961 \text { \& no } \\
\text { further record }\end{array}$ & no & $\begin{array}{l}\text { arrived as Rauwolfia } \\
\text { canescens, one of } 15+ \\
\text { synonyms }\end{array}$ \\
\hline & & Tsukuba, 1983 & 1984 & no & $15+$ synonyms \\
\hline & $\begin{array}{l}\text { Stephanotis floribunda } \\
\text { Brongn. }\end{array}$ & Karlsruhe, 1963 & 1964 & no & $\begin{array}{l}2 \text { more accepted names; } 8 \\
\text { synonyms }\end{array}$ \\
\hline & & $\begin{array}{l}\text { Wuppertal, } 1989 \\
\& 1990\end{array}$ & $\begin{array}{l}2003(2010) \& \\
2007(2008)\end{array}$ & no & \\
\hline & $\begin{array}{l}\text { Thevetia peruviana } \mathrm{K} . \\
\text { Schum. }\end{array}$ & nn, 1989 & 1996 & no & $\begin{array}{l}\text { accepted name is also } \\
\text { Cascabela thevetia; } 10 \\
\text { synonyms }\end{array}$ \\
\hline
\end{tabular}




\begin{tabular}{|c|c|c|c|c|c|}
\hline & $\begin{array}{l}\text { Tweedia coerulea D. } \\
\text { Don ex Sweet }\end{array}$ & Chelsea, 1954 & 1961 & no & $\begin{array}{l}\text { accepted name is also } \\
\text { Oxypetalum coeruleum, } 2 \\
\text { synonyms }\end{array}$ \\
\hline & & Lyon, 1963 & $\begin{array}{l}\text { no further } \\
\text { record }\end{array}$ & no & \\
\hline & $\begin{array}{l}\text { Wrightia religiosa } \\
\text { (Teijsm. \& Binn.) } \\
\text { Benth ex Kurz }\end{array}$ & Bogor, 2004 & 2005 & no & $\begin{array}{l}\text { author acc. to Plant List; } 2 \\
\text { synonyms }\end{array}$ \\
\hline \multirow[t]{5}{*}{ Araliaceae } & $\begin{array}{l}\text { Acanthopanax } \\
\text { setchuenensis Harms } \\
\text { ex Diels }\end{array}$ & Prague, 1962 & 1963 & no & $\begin{array}{l}2 \text { more accepted names; } 4 \\
\text { synonyms }\end{array}$ \\
\hline & \begin{tabular}{|l|} 
X Fatshedera lizei \\
(Cochet) Guillaumin \\
'Silver Prince'
\end{tabular} & $\begin{array}{l}\text { “Lada” Nursery } \\
\text { Slovenia, } 1964\end{array}$ & 1988 & no & $\begin{array}{l}\text { hybrid genus of Fatsia } \\
\text { japonica 'Moserii' and } \\
\text { Hedera helix }\end{array}$ \\
\hline & $\begin{array}{l}\text { Schefflera digitata J.R. } \\
\text { Forst. \& G. Forst. }\end{array}$ & Bucharest, 1985 & 2005 & yes & \\
\hline & & nn, 1986 & 1988 & yes & \\
\hline & $\begin{array}{l}\text { Tetrapanax papyrifer } \\
\text { (Hook.) K. Koch }\end{array}$ & Barcelona, 1968 & 1970 & no & 4 synonyms \\
\hline \multirow[t]{3}{*}{ Asteraceae } & $\begin{array}{l}\text { Chrysocoma } \\
\text { coma-aurea L. }\end{array}$ & $\begin{array}{l}\text { Wuppertal, 1985, } \\
\text { 1986 \& } 1989\end{array}$ & $\begin{array}{l}1988,1987 \& \\
1989\end{array}$ & no & \\
\hline & & Lisbon, 1988 & 1989 & no & \\
\hline & $\begin{array}{l}\text { Rhodanthemum } \\
\text { gayanum (Coss. \& } \\
\text { Durieu) B.H.Wilcox, } \\
\text { K.Bremer \& } \\
\text { Humphries }\end{array}$ & Reading, 2002 & 2004 (2005) & no & $\begin{array}{l}\text { (acc. to IPNI \& Plant List: } \\
\text { unknown to Tropicos) }\end{array}$ \\
\hline \multirow[t]{3}{*}{ Balsaminaceae } & $\begin{array}{l}\text { Impatiens sodenii } \\
\text { Engl. \& Warb. }\end{array}$ & Bonn, 1960 & 2002 & no & 6 synonyms \\
\hline & $\begin{array}{l}\text { Impatiens flaccida } \\
\text { Arn. }\end{array}$ & Bonn, 1972 & $\begin{array}{l}\text { no further } \\
\text { record }\end{array}$ & no & \\
\hline & $\begin{array}{l}\text { Impatiens holstii Engl. } \\
\text { \& Warb. 'Roodkapje' }\end{array}$ & Nijmegen, 1969 & 1972 & no & $\begin{array}{l}\text { accepted species-name is } \\
\text { also } I \text {. walleriana; } 7 \\
\text { synonyms }\end{array}$ \\
\hline \multirow[t]{10}{*}{ Begoniaceae } & $\begin{array}{l}\text { Begonia } \times \text { albopicta } \mathrm{W} \text {. } \\
\text { Bull }\end{array}$ & nn, 1961 & $\begin{array}{l}\text { no further } \\
\text { record }\end{array}$ & no & $\begin{array}{l}\text { there is also B. albopicta W. } \\
\text { Bull }\end{array}$ \\
\hline & $\begin{array}{l}\text { Begonia } \times \text { argenteogut- } \\
\text { tata } \mathrm{M} . \text { Lemoine }\end{array}$ & $\mathrm{nn}, 2000$ & 2003 & no & $\begin{array}{l}\text { purchased as Begonia } \\
\text { 'Argenteoguttata' }\end{array}$ \\
\hline & $\begin{array}{l}\text { Begonia angularis } \\
\text { Raddi }\end{array}$ & Nantes, 1962 & 1963 & no & \\
\hline & $\begin{array}{l}\text { Begonia } \times \text { bunchii L.H. } \\
\text { Bailey }\end{array}$ & Bergen, 1976 & 1988 & no & \\
\hline & $\begin{array}{l}\text { Begonia biserrata } \\
\text { Lindl. }\end{array}$ & Pisa, 1966 & 2013 & no & $\begin{array}{l}\text { arrived as B. palmaris, a } \\
\text { single synonym }\end{array}$ \\
\hline & $\begin{array}{l}\text { Begonia bowerae } \\
\text { Ziesenh. 'Nigra' }\end{array}$ & Lublin, 1987 & 2013 & no & \\
\hline & $\begin{array}{l}\text { Begonia } \times \text { credneri } \mathrm{F} \text {. } \\
\text { Haage \& E. Schmidt }\end{array}$ & Edinburgh, 1960 & 1966 & no & \\
\hline & Begonia caffra Meisn. & Potsdam, 1986 & 1991 & no & $\begin{array}{l}\text { accepted name is also } B \text {. } \\
\text { homonyma }\end{array}$ \\
\hline & $\begin{array}{l}\text { Begonia 'Comte de } \\
\text { Lesseps' }\end{array}$ & Nancy, 1958 & 1961 & no & $\begin{array}{l}\text { arrived as B. 'Ferdinand de } \\
\text { Lesseps'; hybrid }\end{array}$ \\
\hline & $\begin{array}{l}\text { Begonia concinna } \\
\text { Schott }\end{array}$ & Wuppertal, 1978 & $\begin{array}{l}\text { no further } \\
\text { record }\end{array}$ & no & \\
\hline
\end{tabular}




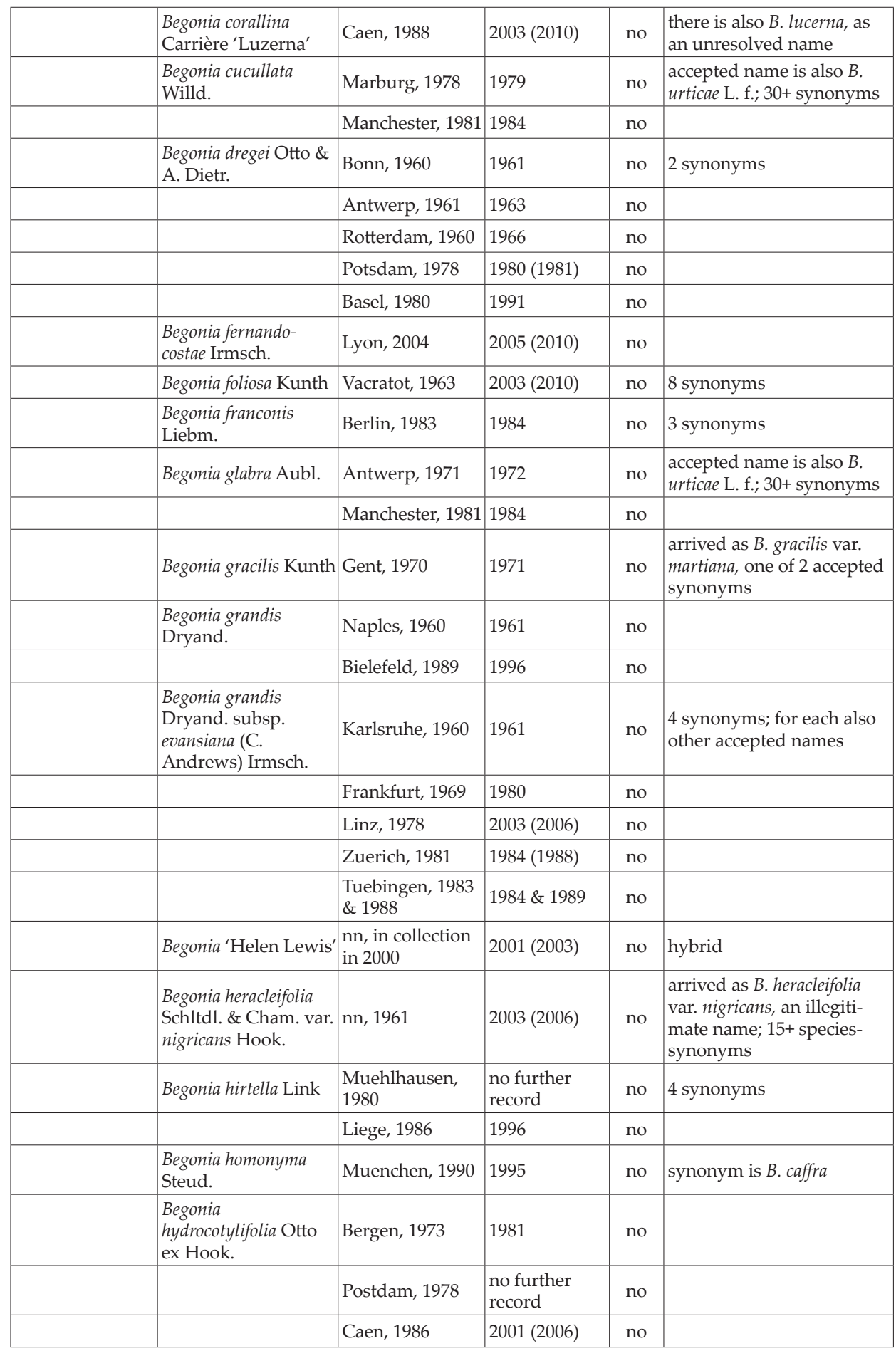




\begin{tabular}{|c|c|c|c|c|c|}
\hline & $\begin{array}{l}\text { Begonia imperialis } \\
\text { Lem. var. smaragdina } \\
\text { Lem. }\end{array}$ & nn, 1961 & 2003 (2006) & no & $\begin{array}{l}3 \text { species-synonyms, } \\
\text { accepted name is also } B \text {. } \\
\text { imperialis; arrived as } \\
\text { 'Smaragdina' }\end{array}$ \\
\hline & $\begin{array}{l}\text { Begonia incarnata } \\
\text { Link \& Otto var. } \\
\text { maculosa (?) }\end{array}$ & Antwerp, 1965 & 1966 & no & unknown variety \\
\hline & $\begin{array}{l}\text { Begonia maculata } \\
\text { Raddi }\end{array}$ & Antwerp, 1966 & 1969 & no & 3 synonyms \\
\hline & $\begin{array}{l}\text { Begonia malabarica } \\
\text { Lam. }\end{array}$ & Bergen, 1965 & 1966 & no & 2 homonyms \\
\hline & & Antwerp, 1965 & 1966 & no & \\
\hline & & Wuppertal, 1978 & $\begin{array}{l}1980 \\
\text { ("incorrect!") }\end{array}$ & no & \\
\hline & & Gembloux, 1989 & 1991 (1995) & no & \\
\hline & $\begin{array}{l}\text { Begonia manicata } \\
\text { Brongn. ex F. Cels } \\
\text { 'Crispa' (?) }\end{array}$ & nn, 1961 & 1975 & no & $\begin{array}{l}\text { arrived as } B . m \text {. var. crispa } \\
\text { hort.; unknown variety }\end{array}$ \\
\hline & $\begin{array}{l}\text { Begonia manicata } \\
\text { Brongn. ex F. Cels } \\
\text { var. aureomaculata } \\
\text { Ziesenh. }\end{array}$ & Antwerp, 1967 & 2003 (2006) & no & $\begin{array}{l}8 \text { species-synonyms; } \\
\text { accepted name is also } B \text {. } \\
\text { manicata; there is also a } \\
\text { cultivar 'Aureo-maculata' }\end{array}$ \\
\hline & $\begin{array}{l}\text { Begonia masoniana } \\
\text { Irmsch. ex Ziesenh. } \\
\text { 'Iron Cross' }\end{array}$ & $\begin{array}{l}\text { "Lada" Nursery } \\
\text { Slovenia, } 1964\end{array}$ & 2012 & no & $\begin{array}{l}\text { also as B. 'Iron Cross' } \\
\text { (hort.) }\end{array}$ \\
\hline & $\begin{array}{l}\text { Begonia molleri (A. } \\
\text { DC.) Warb. }\end{array}$ & Porto, 1967 & 1968 & no & \\
\hline & $\begin{array}{l}\text { Begonia mollicaulis } \\
\text { Irmsch. }\end{array}$ & Gent, 1988 & 1996 & no & \\
\hline & $\begin{array}{l}\text { Begonia multiflora } \\
\text { Benth. 'Helen Harms' }\end{array}$ & nn, 1962 & 1968 & no & arrived as B. 'Helen Harms' \\
\hline & $\begin{array}{l}\text { Begonia multiflora } \\
\text { Benth. 'Plam Deyon' } \\
(?)\end{array}$ & nn, 1962 & 1968 & no & unknown cultivar \\
\hline & $\begin{array}{l}\text { Begonia oxysperma A. } \\
\text { DC. }\end{array}$ & Vienna, 2003 & 2005 & no & \\
\hline & Begonia pearcei Hook. & Montreal, 1960 & 1963 & no & \\
\hline & $\begin{array}{l}\text { Begonia 'Perle de } \\
\text { Lorraine' }\end{array}$ & nn, 1961 & $\begin{array}{l}\text { no further } \\
\text { record }\end{array}$ & no & hybrid \\
\hline & Begonia rajah Ridl. & Gent, 1988 & 1996 & no & \\
\hline & Begonia Rex Cultorum & nn, 1961 & $\begin{array}{l}\text { no further } \\
\text { record }\end{array}$ & no & $\begin{array}{l}\text { there is also described a } \\
\text { wild species B. rex Putz, } \\
\text { but the large group of } \\
\text { cultivars 'Rex Cultorum' } \\
\text { are horticultural hybrids } \\
\text { (acc. to UK Begonia } \\
\text { Society) }\end{array}$ \\
\hline & & Vacratot, 1963 & 1966 & no & \\
\hline & & nn, 1980 & 1988 & no & \\
\hline & & nn, 1987 & 1995 & no & \\
\hline & & nn, 1987 & 1995 & no & \\
\hline & $\begin{array}{l}\text { Begonia Rex Cultorum } \\
\text { 'Abel Carriere' }\end{array}$ & Manchester, 1985 & 1996 & no & \\
\hline
\end{tabular}




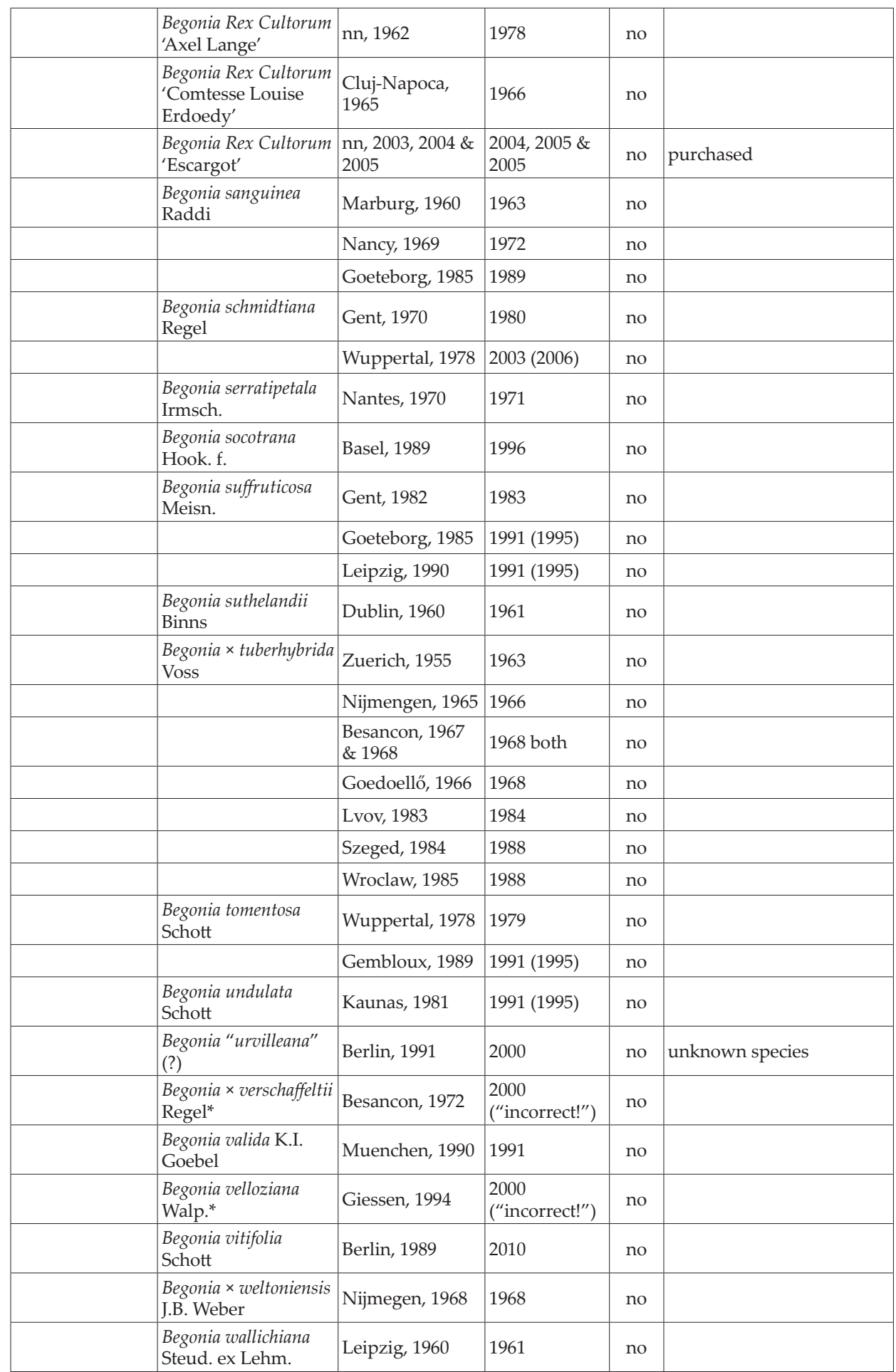




\begin{tabular}{|c|c|c|c|c|c|}
\hline & & Potsdam, 1978 & $\begin{array}{l}\text { no further } \\
\text { record }\end{array}$ & no & \\
\hline & & Wuppertal, 1987 & 1988 & no & \\
\hline \multirow[t]{12}{*}{ Bignoniaceae } & $\begin{array}{l}\text { Jacaranda acutifolia } \\
\text { Bonpl. 'Tango' (?) }\end{array}$ & Frankfurt, 1969 & 1989 & no & unknown cultivar \\
\hline & $\begin{array}{l}\text { Jacaranda mimosifolia } \\
\text { D. Don }\end{array}$ & Bogor, 1980 & 1981 & no & $\begin{array}{l}\text { arrived as J.filicifolia, one of } \\
3 \text { accepted names }\end{array}$ \\
\hline & & Legon, 1964 & 1984 & no & 4 synonyms \\
\hline & & Izmir, 1965 & 1978 & no & \\
\hline & & Frankfurt, 1969 & 1989 & no & \\
\hline & & Hong Kong, 1979 & 1989 & no & \\
\hline & $\begin{array}{l}\text { Macfadyena } \\
\text { unguis-cati (L.) A.H. } \\
\text { Gentry }\end{array}$ & nn, 1961 & 1968 & no & $\begin{array}{l}\text { arrived as Bignonia } \\
\text { tweediana; } 40+\text { accepted } \\
\text { names and synonyms }\end{array}$ \\
\hline & & Bonn, 1981 & 1983 & no & $\begin{array}{l}\text { from a native locality in the } \\
\text { Caribbean; } 40+\text { accepted } \\
\text { names and synonyms }\end{array}$ \\
\hline & & Adelaide, 1982 & 1988 & no & $\begin{array}{l}\text { arrived as Doxantha } u .-c . ; \\
\text { 40+ accepted names and } \\
\text { synonyms }\end{array}$ \\
\hline & $\begin{array}{l}\text { Markhamia lutea } \\
\text { (Benth.) K. Schum. }\end{array}$ & $\begin{array}{l}\text { Orotava- } \\
\text { Tenerife, } 2004\end{array}$ & 2010 & no & \\
\hline & $\begin{array}{l}\text { Pandorea ricasoliana } \\
\text { (Tanfani) K. Schum. }\end{array}$ & Ariana, 1963 & 1978 & no & \\
\hline & $\begin{array}{l}\text { Radermachera sinica } \\
\text { (Hance) Hemsl. }\end{array}$ & nn, 2003 & 2005 & no & purchased; 2 synonyms \\
\hline \multirow[t]{2}{*}{ Boraginaceae } & $\begin{array}{l}\text { Wigandia urens (Ruiz } \\
\text { \& Pav.) Kunth }\end{array}$ & Stuttgart, 1987 & 1990 & no & $\begin{array}{l}\text { arrived as } W . \text { caracasana, } \\
\text { one of } 15+\text { synonyms }\end{array}$ \\
\hline & & \begin{tabular}{|l} 
Duesseldorf, \\
1989
\end{tabular} & 1990 & no & $15+$ synonyms \\
\hline \multirow[t]{9}{*}{ Campanulaceae } & $\begin{array}{l}\text { Azorina vidalii } \\
\text { (H.C.Wats.) Feer }\end{array}$ & \begin{tabular}{|l|} 
Orotava- \\
Tenerife, 2004 \\
\end{tabular} & 2006 & no & relocated \\
\hline & $\begin{array}{l}\text { Campanumoea } \\
\text { javanica Blume }\end{array}$ & Shangai, 2003 & $2004(2010)$ & no & syn. Codonopsis javanica \\
\hline & $\begin{array}{l}\text { Hippobroma longiflora } \\
\text { (L.) G. Don }\end{array}$ & Bergen, 1965 & 1968 & no & $\begin{array}{l}\text { arrived as Isotoma } 1 . \\
\text { (invalid); } 7 \text { synonyms }\end{array}$ \\
\hline & & Bonn, 1980 & 1981 & no & 7 synonyms \\
\hline & & Nancy, 2002 & $2010(2012)$ & no & \\
\hline & $\begin{array}{l}\text { Laurentia gasparrinii } \\
\text { (Tineo) Strobl }\end{array}$ & $\begin{array}{l}\text { Copenhagen, } \\
1978 \\
\end{array}$ & 1979 (1989) & no & \\
\hline & Lobelia fulgens Willd. & Karlsruhe, 2002 & 2005 & no & $\begin{array}{l}\text { syn. L. cardinalis; } 20 \\
\text { synonyms }\end{array}$ \\
\hline & $\begin{array}{l}\text { Michauxia campanu- } \\
\text { loides L'Hér. }\end{array}$ & Teplice, 2004 & 2005 & no & \\
\hline & $\begin{array}{l}\text { Musschia aurea (L.) } \\
\text { Dumort. }\end{array}$ & Bochum, 2002 & $2003(2005)$ & no & \\
\hline Convolvulaceae & $\begin{array}{l}\text { Ipomoea batatas (L.) } \\
\text { Lam. }\end{array}$ & Wroclaw, 1986 & $2002(2010)$ & no & 30 synonyms \\
\hline \multirow[t]{2}{*}{ Cucurbitaceae } & $\begin{array}{l}\text { Schizopepon } \\
\text { bryoniifolius Maxim. }\end{array}$ & Tskuba, 2002 & 2004 (2006) & no & 2 synonyms \\
\hline & $\begin{array}{l}\text { Trichosanthes } \\
\text { quinquangulata A. } \\
\text { Gray }\end{array}$ & Hengchun, 2002 & 2003 & no & \\
\hline
\end{tabular}




\begin{tabular}{|c|c|c|c|c|c|}
\hline & $\begin{array}{l}\text { Momordica charantia } \\
\text { L. }\end{array}$ & Duisburg, 1969 & 1970 & yes & $10+$ synonyms \\
\hline Erythroxylaceae & $\begin{array}{l}\text { Erythroxylum } \\
\text { novogranatense (D. } \\
\text { Morris) Hieron. }\end{array}$ & Singapore, 1961 & 1984 & no & 4 synonyms \\
\hline \multirow[t]{4}{*}{ Euphorbiaceae } & $\begin{array}{l}\text { Dalechampia } \\
\text { spathulata (Scheidw.) } \\
\text { Baill. var. spathulata } \\
(?)\end{array}$ & Tuebingen, 1963 & 1968 & no & $\begin{array}{l}\text { unknown variety; with a } \\
\text { syn. D. roezliana var. rosea } \\
\text { - acc. to Tropicos, these are } \\
\text { separate species; } 8 \\
\text { synonyms }\end{array}$ \\
\hline & $\begin{array}{l}\text { Euphorbia } \\
\text { tithymaloides (L.) Poit. }\end{array}$ & Antwerp, 1964 & 1966 & no & $\begin{array}{l}\text { accepted name is also } \\
\text { Pedilanthus t.; } 30+ \\
\text { synonyms }\end{array}$ \\
\hline & & $\begin{array}{l}\text { Vacratot, } 1963 \& \\
1965\end{array}$ & 1968 both & yes & \\
\hline & $\begin{array}{l}\text { Triadica sebifera }(\mathrm{L} .) \\
\text { Small }\end{array}$ & Sendai, 1980 & 1991 & no & $\begin{array}{l}\text { accepted name is also } \\
\text { Sapium sebiferum; } 10+ \\
\text { synonyms }\end{array}$ \\
\hline \multirow[t]{15}{*}{ Fabaceae } & $\begin{array}{l}\text { Caesalpinia } \\
\text { pulcherrima (L.) Sw. } \\
\text { 'Ruby Red' }\end{array}$ & Auroville, 1990 & 1996 & no & $\begin{array}{l}\text { accepted name is also } \\
\text { Poinciana pulcherrima; } 6 \\
\text { species-synonyms }\end{array}$ \\
\hline & $\begin{array}{l}\text { Cajanus cajan (L.) } \\
\text { Huth }\end{array}$ & $\begin{array}{l}\text { Duesseldorf, } \\
2012\end{array}$ & 2013 & no & $\begin{array}{l}\text { several accepted names } \\
\text { and many synonyms }\end{array}$ \\
\hline & $\begin{array}{l}\text { Desmodium illinoense } \\
\text { A. Gray }\end{array}$ & Lyon, 1991 & 1996 & no & \\
\hline & $\begin{array}{l}\text { Gastrolobium } \\
\text { coriaceum (Sm.) G. } \\
\text { Chandler \& Crisp } \\
\end{array}$ & Prague, 1961 & 1965 & no & $\begin{array}{l}\text { arrived as Oxylobium } \\
\text { retusum; one of } 10 \\
\text { synonyms }\end{array}$ \\
\hline & $\begin{array}{l}\text { Hardenbergia } \\
\text { comptoniana Benth. }\end{array}$ & Menton, 2011 & 2013 & no & \\
\hline & $\begin{array}{l}\text { Leucaena glauca } \\
\text { Benth. }\end{array}$ & Basel, 1995 & 1996 & yes & $\begin{array}{l}3 \text { more accepted names; } 7 \\
\text { synonyms }\end{array}$ \\
\hline & $\begin{array}{l}\text { Samanea saman (Jacq.) } \\
\text { Merr. }\end{array}$ & Bogor, 1984 & $1984(1989)$ & no & $\begin{array}{l}3 \text { more accepted names; } 6 \\
\text { synonyms }\end{array}$ \\
\hline & & Auroville, 1990 & 1996 & no & \\
\hline & Tamarindus indica L. & Brisbane, 1963 & 1964 & no & $\begin{array}{l}\text { monotypic genus or with } \\
\text { several species? Widely } \\
\text { cultivated; } 3 \text { synonyms }\end{array}$ \\
\hline & & Legon, 1970 & 1989 & no & \\
\hline & & Bogor, 1978 & 1984 & no & \\
\hline & $\begin{array}{l}\text { Tipuana tipu (Benth.) } \\
\text { Kuntze }\end{array}$ & $\begin{array}{l}\text { Braunschweig, } \\
1978 \text { \& } 1979\end{array}$ & $1989 \& 1979$ & yes & $\begin{array}{l}\text { arrived as T. speciosa } \\
\text { Benth., one of } 3 \text { synonyms }\end{array}$ \\
\hline & & $\begin{array}{l}\text { Blanes, } 1981 \& \\
1984\end{array}$ & $1984 \& 1989$ & yes & 3 synonyms \\
\hline & & $\begin{array}{l}\text { Barcelona, } 1981 \\
\& 1982\end{array}$ & $1989 \& 1984$ & yes & \\
\hline & & Coimbra, 1984 & 1989 & yes & \\
\hline \multirow[t]{5}{*}{ Gesneriaceae } & $\begin{array}{l}\text { Achimenes erecta } \\
\text { (Lam.) H.P. Fuchs }\end{array}$ & Duisburg 1982 & $\begin{array}{l}\text { no further } \\
\text { record }\end{array}$ & yes & 10 synonyms \\
\hline & & Berlin, 2003 & 2005 (2006) & yes & \\
\hline & $\begin{array}{l}\text { Aeschynanthus } \\
\text { lobbianus Hook. }\end{array}$ & Bergen, 1957 & 1961 & yes & \\
\hline & & Antwerp, 1972 & 1980 & yes & \\
\hline & & nn, $2001 \& 2003$ & $2005 \& 2007$ & yes & purchased \\
\hline
\end{tabular}




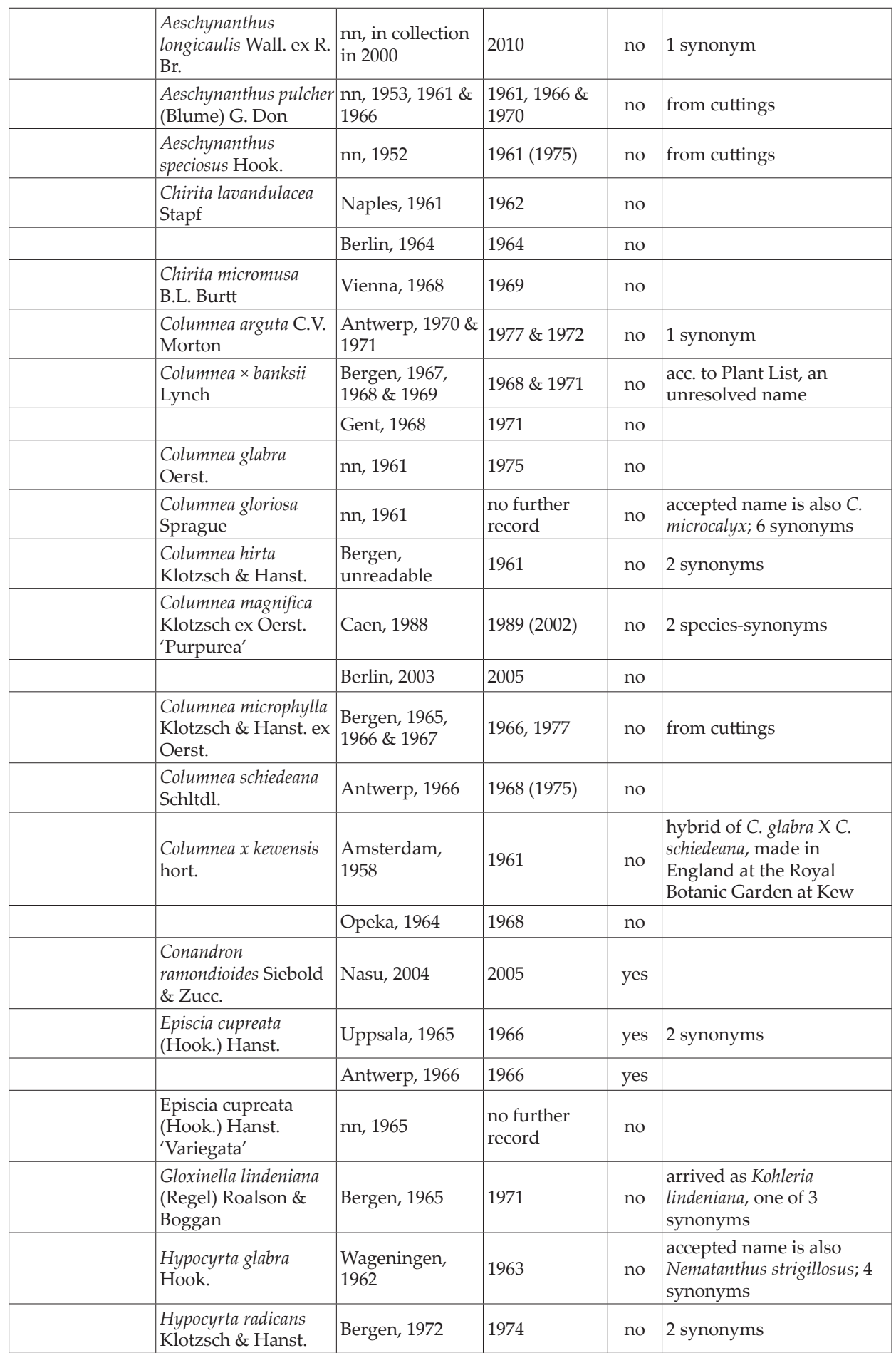




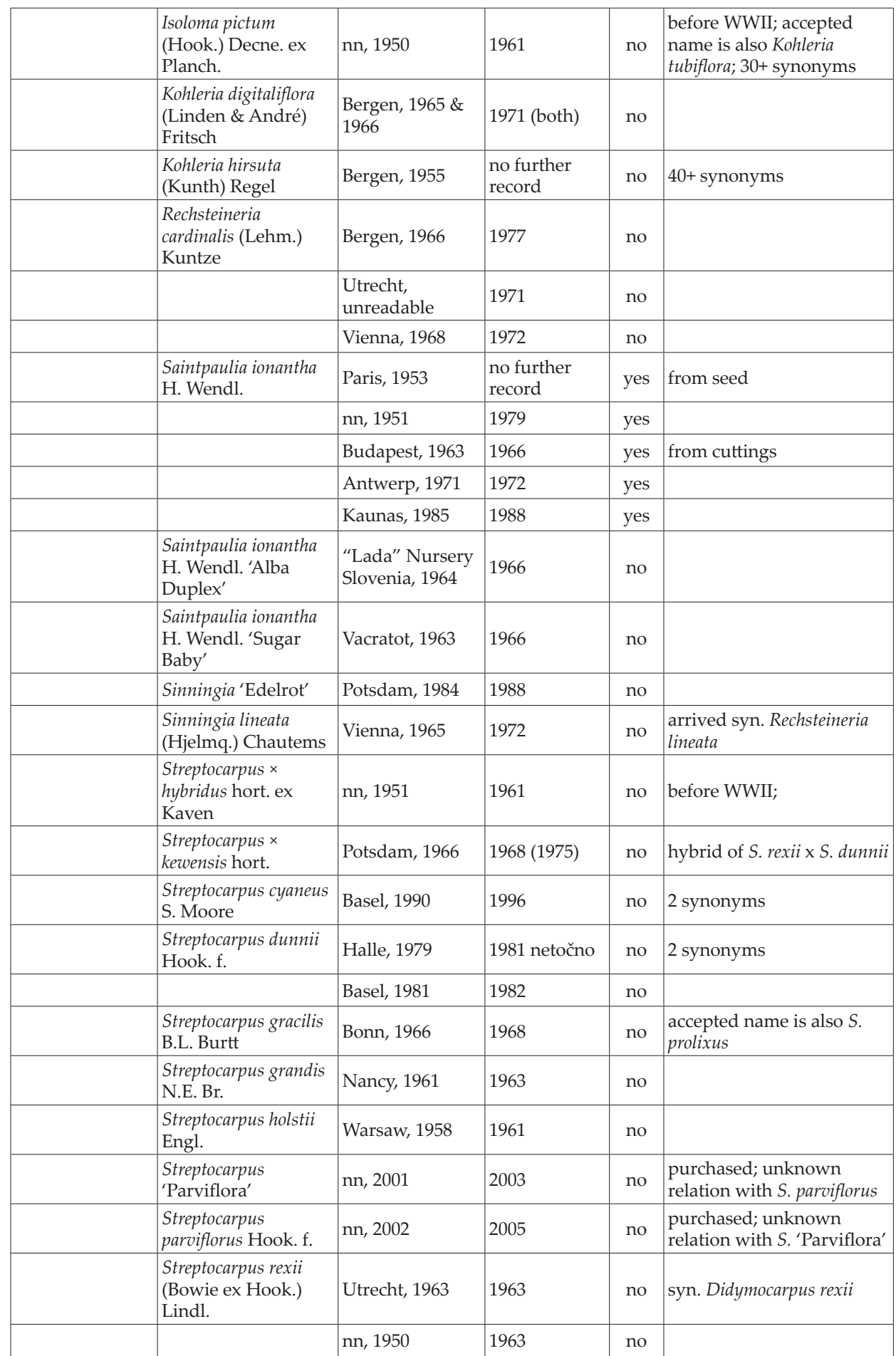




\begin{tabular}{|c|c|c|c|c|c|}
\hline & & Cork, 1950 & 1981 & no & \\
\hline & & Košice, 1957 & 1963 & no & \\
\hline & & Warsaw, 1958 & 1963 & no & \\
\hline & & Stockholm, 1963 & 1963 & no & \\
\hline & & Berlin, 1966 & 1971 & no & \\
\hline & & Muenster, 1966 & 1969 & no & \\
\hline & & Meinz, 1967 & 1979 & no & \\
\hline & & Dresden, 1967 & 1995 & no & \\
\hline & & $\begin{array}{l}\text { Potsdam, } 1967 \& \text { \& } \\
1978\end{array}$ & $\begin{array}{l}1995 \& 2005 \\
(2006)\end{array}$ & no & \\
\hline & & Nancy, 1971 & 1980 & no & \\
\hline & \begin{tabular}{|l|} 
Streptocarpus rexii \\
(Bowie ex Hook.) \\
Lindl. var. pelona (?) \\
\end{tabular} & Kaunas, 1967 & 1968 & no & unknown variety \\
\hline & $\begin{array}{l}\text { Streptocarpus saxorum } \\
\text { Engl. }\end{array}$ & nn, 2002 & 2005 & no & purchased \\
\hline & \begin{tabular}{|l|} 
Streptocarpus wilmsii \\
Engl.
\end{tabular} & Berlin, 1964 & 1966 (1975) & no & syn. S. muddii \\
\hline \multirow[t]{17}{*}{ Lamiaceae } & $\begin{array}{l}\text { Clerodendrum } \\
\text { cunninghamii Benth. }\end{array}$ & Brisbane, 1987 & 1991 (1995) & no & $\begin{array}{l}\text { the genus previously } \\
\text { belonged to fam. } \\
\text { Verbenaceae }\end{array}$ \\
\hline & $\begin{array}{l}\text { Clerodendrum fallax } \\
\text { Lindl.* }\end{array}$ & Bergen, 1958 & $\begin{array}{l}1961 \\
\text { ("incorrect"!) }\end{array}$ & no & $\begin{array}{l}\text { arrived as C. speciosissi- } \\
\text { mum, probably hort.; fam. } \\
\text { Verbenaceae }\end{array}$ \\
\hline & & Liberec, 1988 & $\begin{array}{l}1991 \\
\text { ("incorrect!") }\end{array}$ & no & \\
\hline & $\begin{array}{l}\text { Clerodendrum } \\
\text { fortunatum L. }\end{array}$ & Shangai, 1988 & 1988 & no & 6 synonyms \\
\hline & $\begin{array}{l}\text { Clerodendrum } \\
\text { kaichianum P.S. Hsu }\end{array}$ & Shangai, 1991 & 1995 & no & \\
\hline & $\begin{array}{l}\text { Clerodendrum } \\
\text { speciosissimum C. } \\
\text { Morren }\end{array}$ & Goettingen, 1979 & 1980 & no & \\
\hline & & Bergen, 1973 & $2002(2011)$ & no & \\
\hline & & Berlin, 1973 & 1986 & no & \\
\hline & & $\begin{array}{l}\text { Amsterdam, } \\
1979\end{array}$ & 1986 (1995) & no & \\
\hline & & $\begin{array}{l}\text { Leningrad (St. } \\
\text { Petersburg), } 1973\end{array}$ & 1978 & no & \\
\hline & & Basel, 1973 & 1978 & no & \\
\hline & & Muenchen, 1978 & 1991 (1995) & no & \\
\hline & $\begin{array}{l}\text { Clerodendrum } \\
\text { squamatum Vahl }\end{array}$ & Rouen, 1988 & 1989 & no & $\begin{array}{l}\text { accepted name is also } C \text {. } \\
\text { japonicum; } 9 \text { synonyms }\end{array}$ \\
\hline & $\begin{array}{l}\text { Clerodendrum } \\
\text { thomsoniae Balf. }\end{array}$ & nn, 2000 & 2005 (2005) & no & $\begin{array}{l}\text { purchased as C. thomsonae, } \\
\text { fam. Verbenaceae }\end{array}$ \\
\hline & $\begin{array}{l}\text { Clerodendrum } \\
\text { umbellatum Poir. }\end{array}$ & Marburg, 1986 & 1988 & no & \\
\hline & $\begin{array}{l}\text { Plectranthus fruticosus } \\
\text { L'Hér. }\end{array}$ & $\begin{array}{l}\text { Nancy, 1971, } \\
\text { 1976, 1980, } 1982\end{array}$ & \begin{tabular}{|l} 
1980, 1984, \\
1980, 2001 \\
("incorrect!")
\end{tabular} & yes & \\
\hline & & nn, 1961 & 1963 & yes & \\
\hline
\end{tabular}




\begin{tabular}{|c|c|c|c|c|c|}
\hline & $\begin{array}{l}\text { Plectranthus } \\
\text { oertendahlii T.C.E. Fr. }\end{array}$ & nn, 1961 & 1984 & no & \\
\hline & $\begin{array}{l}\text { Plectranthus } \\
\text { tomentosus Benth.* }\end{array}$ & Oxford, 1983 & $\begin{array}{l}2000 \\
\text { ("incorrect!") }\end{array}$ & no & \\
\hline Lecythidaceae & $\begin{array}{l}\text { Barringtonia samoensis } \\
\text { A. Gray }\end{array}$ & Bogor, 2004 & 2010 & no & \\
\hline \multirow[t]{24}{*}{ Malvaceae } & $\begin{array}{l}\text { Abutilon hybridum } \\
\text { hort. ex Voss }\end{array}$ & Hamburg, 1960 & 1963 & no & \\
\hline & & Bratislava, 1972 & 1975 & no & \\
\hline & $\begin{array}{l}\text { Abutilon indicum (L.) } \\
\text { Sweet }\end{array}$ & Berlin, 1973 & 1976 & no & $10+$ synonyms \\
\hline & & Turku, 1985 & 1996 & no & \\
\hline & & Haren, 1989 & 2003 & no & \\
\hline & $\begin{array}{l}\text { Abutilon mauritianum } \\
\text { (Jacq.) Medik. }\end{array}$ & Haren, 1989 & $\begin{array}{l}\text { no further } \\
\text { record }\end{array}$ & no & \\
\hline & \begin{tabular}{|l|} 
Abutilon \\
sonneratianum (Cav.) \\
Sweet
\end{tabular} & $\begin{array}{l}\text { Haren, } 1983 \& \\
1987\end{array}$ & $1991 \& 1994$ & no & \\
\hline & $\begin{array}{l}\text { Abutilon theophrasti } \\
\text { Medik. }\end{array}$ & Marburg, 1986 & 1989 & no & $10+$ synonyms \\
\hline & $\begin{array}{l}\text { Abutilon vitifolium } \\
\text { (Cav.) G. Don }\end{array}$ & $\begin{array}{l}\text { Harrogate \& } \\
\text { Manchester, } 1989\end{array}$ & 1989 & no & $\begin{array}{l}\text { accepted name is also } \\
\text { Corynabutilon vitifolium; } 3 \\
\text { synonyms }\end{array}$ \\
\hline & Adansonia digitata $\mathrm{L}$. & $\begin{array}{l}\text { Kirstenbosch, } \\
1986 \\
\end{array}$ & $2008(2010)$ & no & 8 synonyms \\
\hline & & Strasbourg, 1985 & 1986 & no & \\
\hline & $\begin{array}{l}\text { Hibiscus calyphyllus } \\
\text { Cav. }\end{array}$ & Harren, 1989 & 1991 & no & syn. H. calycinus \\
\hline & & $\begin{array}{l}\text { nn, in collection } \\
\text { in } 2000\end{array}$ & 2005 & yes & \\
\hline & $\begin{array}{l}\text { Hibiscus cameronii } \\
\text { Knowles \& Westcott }\end{array}$ & Harren, 1989 & 1991 & no & 2 synonyms \\
\hline & $\begin{array}{l}\text { Hibiscus eetveldeanus } \\
\text { De Wild. \& Durand* }\end{array}$ & Berlin, 1963 & $\begin{array}{l}1963 \\
(\text { "incorrect!") }\end{array}$ & no & syn. H. acetosella \\
\hline & $\begin{array}{l}\text { Hibiscus moscheutos } \\
\text { L. }\end{array}$ & Karlsruhe, 1986 & 1989 (1991) & no & \\
\hline & Hibiscus mutabilis L. & Siene, 1989 & 1991 & no & 5 synonyms \\
\hline & & Kunming, 1989 & 1996 & no & \\
\hline & Hibiscus roseus Thore & Paris, 1988 & 1989 & no & \\
\hline & Hibiscus sabdariffa L. & Potsdam, 1990 & 1996 & no & 5 synonyms \\
\hline & & Joensuu, 1989 & 1989 & no & \\
\hline & Sida rhombifolia L. & Bruxelles, 1962 & 1963 ( 1989) & no & $20+$ synonyms \\
\hline & $\begin{array}{l}\text { Thespesia lampas } \\
\text { (Cav.) Dalzell \& A. } \\
\text { Gibson }\end{array}$ & Haren, 1984 & $\begin{array}{l}\text { no further } \\
\text { record }\end{array}$ & no & 3 synonyms \\
\hline & $\begin{array}{l}\text { Thespesia populnea } \\
\text { (L.) Sol. ex Corrêa }\end{array}$ & Bonn, 1981 & 1984 (1989) & no & $\begin{array}{l}\text { accepted name also } T \text {. } \\
\text { populneoides; } 10+\text { synonyms }\end{array}$ \\
\hline \multirow[t]{3}{*}{ Melastomataceae } & $\begin{array}{l}\text { Bertolonia maculata } \\
\text { DC. }\end{array}$ & Basel, 1981 & 1989 & no & \\
\hline & & Karlsruhe, 1984 & 1989 & no & \\
\hline & Calvoa orientalis Taub. & Berlin, 1984 & $1984(2002)$ & no & syn. C. sessiliflora \\
\hline
\end{tabular}




\begin{tabular}{|c|c|c|c|c|c|}
\hline & $\begin{array}{l}\text { Gravesia guttata } \\
\text { (Hook.) Triana }\end{array}$ & Bergen, 1981 & 1981 (1989) & no & syn. Bertolonia g. \\
\hline & $\begin{array}{l}\text { Medinilla magnifica } \\
\text { Lindl. }\end{array}$ & nn, 2010 & 2010 & no & purchased \\
\hline \multirow[t]{15}{*}{ Moraceae } & $\begin{array}{l}\text { Dorstenia contrajerva } \\
\text { L. var. hudsoniana (?) }\end{array}$ & nn, 1952 & $\begin{array}{l}\text { no further } \\
\text { record }\end{array}$ & no & unknown variety \\
\hline & $\begin{array}{l}\text { Dorstenia contrajerva } \\
\text { L. var. maculata } \\
\text { (Lem.) Bureau }\end{array}$ & Kaunas, 1960 & 1961 & no & \\
\hline & $\begin{array}{l}\text { Ficus elastica Roxb. ex } \\
\text { Hornem. 'Schryveri- } \\
\text { ana' }\end{array}$ & $\begin{array}{l}\text { "Lada" Nursery } \\
\text { Slovenia, } 1964\end{array}$ & 1978 (1989) & no & $\begin{array}{l}3 \text { species-synonyms; that } \\
\text { cultivar is recognized also } \\
\text { as a natural variety, " } F \text {. } \\
\text { decora var. schryveriana" }\end{array}$ \\
\hline & $\begin{array}{l}\text { Ficus elastica Roxb. ex } \\
\text { Hornem. 'Variegata' }\end{array}$ & $\begin{array}{l}\text { nn, in collection } \\
\text { in } 2000\end{array}$ & 2010 & no & $\begin{array}{l}\text { leaf stripes faded } \\
\text { eventually }\end{array}$ \\
\hline & Ficus "hemeana" (?) & $\begin{array}{l}\text { nn, in collection } \\
\text { in } 2000\end{array}$ & 2005 & no & $\begin{array}{l}\text { unknown taxon, maybe } \\
\text { misspeled F. henneana? }\end{array}$ \\
\hline & Ficus henneana Miq. & nn, 1963 & 2000 & no & syn. F. superba var. henneana \\
\hline & Ficus infectoria Roxb. & nn, 1954 & 1961 (1989) & no & $\begin{array}{l}\text { accepted name is also } F . \\
\text { virens }\end{array}$ \\
\hline & & Genova, 1967 & 1989 & no & \\
\hline & $\begin{array}{l}\text { Ficus microphylla } \\
\text { Salzm. ex Miq. }\end{array}$ & Antwerp, 1966 & $1971(1989)$ & no & \\
\hline & $\begin{array}{l}\text { Ficus parcellii Veitch } \\
\text { ex Cogn. \& Marchal }\end{array}$ & Prague, 1964 & $1966(1989)$ & no & $\begin{array}{l}\text { accepted name is also } F \text {. } \\
\text { aspera }\end{array}$ \\
\hline & $\begin{array}{l}\text { Ficus quercifolia } \\
\text { Blume }\end{array}$ & Antwerp, 1965 & 1968 & no & \\
\hline & & Uppsala, 1965 & $1977(1989)$ & no & \\
\hline & Ficus umbellata Vahl & Legon, 1967 & $1981(1989)$ & no & \\
\hline & $\begin{array}{l}\text { Ficus variegata Blume } \\
\text { var. chlorocarpa } \\
\text { (Benth.) Benth. ex } \\
\text { King }\end{array}$ & $\begin{array}{l}\text { Hong Kong, } \\
1973\end{array}$ & $1977(1989)$ & no & $\begin{array}{l}\text { accepted name is also } F \text {. } \\
\text { variegata; known also as } F \text {. } \\
\text { chlorocarpa }\end{array}$ \\
\hline & $\begin{array}{l}\text { Ficus watkinsiana F.M. } \\
\text { Bailey }\end{array}$ & Melbourne, 1959 & 1981 (1989) & no & \\
\hline Oleaceae & $\begin{array}{l}\text { Jasminum sambac (L.) } \\
\text { Aiton }\end{array}$ & Bratislava, 1971 & 1989 & no & syn. Nyctanthes sambac L. \\
\hline Oxalidaceae & Averrhoa carambola L. & Basel, 1990 & $2009(2010)$ & no & 4 synonyms \\
\hline \multirow[t]{4}{*}{ Passifloraceae } & $\begin{array}{l}\text { Passiflora adenopoda } \\
\text { DC. }\end{array}$ & Muenchen, 1962 & 1968 (1989) & no & 7 synonyms \\
\hline & $\begin{array}{l}\text { Passiflora } \\
\text { quadrangularis L. }\end{array}$ & Glasgow, 1953 & $\begin{array}{l}\text { no further } \\
\text { record }\end{array}$ & no & 6 synonyms \\
\hline & & $\begin{array}{l}\text { Wageningen, } \\
1963\end{array}$ & 1968 & no & \\
\hline & & $\begin{array}{l}\text { Bratislava, } 1971 \\
\& 1979\end{array}$ & $\begin{array}{l}1974 \& \text { \& } 1984 \\
(1989)\end{array}$ & no & \\
\hline Pentaphyllaceae & $\begin{array}{l}\text { Cleyera japonica } \\
\text { Thunb. }\end{array}$ & Kyoto, 1986 & $1987(1988)$ & no & $\begin{array}{l}\text { accepted name is also } \\
\text { Ternstroemia } j . ; 8 \text { synonyms; } \\
\text { arrived as fam. Theaceae }\end{array}$ \\
\hline \multirow[t]{2}{*}{ Phytolaccaceae } & $\begin{array}{l}\text { Rivina purpurascens } \\
\text { Schrad. }\end{array}$ & nn, 1974 & 1975 & no & $40+$ synonyms of $R$.humilis \\
\hline & $\begin{array}{l}\text { Rivina tinctoria Ham. } \\
\text { ex G. Don }\end{array}$ & Bruxelles, 1970 & $\begin{array}{l}\text { no further } \\
\text { records }\end{array}$ & no & \\
\hline
\end{tabular}




\begin{tabular}{|c|c|c|c|c|c|}
\hline Piperaceae & $\begin{array}{l}\text { Peperomia argyreia } \\
\text { (Miq.) E. Morren }\end{array}$ & Genova, 1963 & 1969 & no & 2 synonyms \\
\hline & & nn, $1969 \& 1972$ & 1991 both & no & \\
\hline & & Duisburg, 1988 & $2005(2007)$ & no & \\
\hline & $\begin{array}{l}\text { Peperomia bracteata } \\
\text { A.W. Hill }\end{array}$ & nn, 2003 & $2005(2010)$ & no & \\
\hline & $\begin{array}{l}\text { Peperomia caperata } \\
\text { Yunck. }\end{array}$ & $\begin{array}{l}\text { nn, 1961, } 1963 \& \text { \& } \\
1972\end{array}$ & $\begin{array}{l}1969,2005 \& \\
2003\end{array}$ & no & \\
\hline & & Potsdam, 1969 & 1984 & no & \\
\hline & $\begin{array}{l}\text { Peperomia caperata } \\
\text { Yunck. 'Pink Lady' }\end{array}$ & nn, 2003 & 2005 & no & \\
\hline & $\begin{array}{l}\text { Peperomia caperata } \\
\text { Yunck. 'Shumi' }\end{array}$ & nn, 2002 & 2003 & no & \\
\hline & $\begin{array}{l}\text { Peperomia caulibarbis } \\
\text { Miq. }\end{array}$ & nn, 1961 & $\begin{array}{l}\text { no further } \\
\text { record }\end{array}$ & no & $\begin{array}{l}\text { an accepted name between } \\
\text { 30+ synonyms; } P \text {. glabella } \\
\text { and Piper glabellum also are } \\
\text { accepted }\end{array}$ \\
\hline & & Iasi, 1963 & 1969 & no & \\
\hline & \begin{tabular}{|l|} 
Peperomia \\
dolabriformis Kunth $\mathrm{f}$. \\
angustifolia (?)
\end{tabular} & Salaspils, 1988 & 2003 (2005) & no & $\begin{array}{l}\text { unknown form; probably a } \\
\text { cultivar }\end{array}$ \\
\hline & $\begin{array}{l}\text { Peperomia forsythii C. } \\
\text { DC. }\end{array}$ & Basel, 1986 & 1989 & no & $\begin{array}{l}\text { accepted name is also } P \text {. } \\
\text { trichophylla }\end{array}$ \\
\hline & $\begin{array}{l}\text { Peperomia galioides } \\
\text { Kunth }\end{array}$ & Basel, 1988 & 2003 (2005) & no & $35+$ synonyms \\
\hline & $\begin{array}{l}\text { Peperomia } \\
\text { griseoargentia Yunck. }\end{array}$ & $\begin{array}{l}\text { Uppsala, } 1961 \& \text { \& } \\
1964\end{array}$ & $1991 \& 1966$ & no & \\
\hline & & Vacratot, 1963 & 1963 & no & \\
\hline & & nn, 1981 & 2003 (2004) & no & \\
\hline & $\begin{array}{l}\text { Peperomia longispicata } \\
\text { C. DC. 'Variegata'* }\end{array}$ & Antwerp, 1960 & $\begin{array}{l}1981 \\
\text { ("incorrect!") }\end{array}$ & no & $\begin{array}{l}\text { arrived as a natural variety, } \\
\text { "var. variegata" }\end{array}$ \\
\hline & $\begin{array}{l}\text { Peperomia maculosa } \\
\text { (L.) Hook. }\end{array}$ & Gent, 1979 & 2005 & no & $\begin{array}{l}10 \text { synonyms, among } \\
\text { which } P \text {. grandifolia, } P \text {. } \\
\text { sarcophylla etc. are } \\
\text { sometimes considered to } \\
\text { be accepted }\end{array}$ \\
\hline & & $\begin{array}{l}\text { Amsterdam, } \\
1960\end{array}$ & 1963 & no & \\
\hline & & Nancy, 1966 & 1972 & no & \\
\hline & & Basel, 1989 & 1991 & no & \\
\hline & & Gent, 1988 i 1979 & 1995 \& 2010 & no & \\
\hline & & Goettingen, 1993 & 1995 & no & \\
\hline & $\begin{array}{l}\text { Peperomia magnoliifo- } \\
\text { lia (Jacq.) A. Dietr. }\end{array}$ & $\begin{array}{l}\text { Antwerp, } 1962 \& \text { \& } \\
1964\end{array}$ & 1966 (both) & no & $\begin{array}{l}\text { accepted name is also } P \text {. } \\
\text { obtusifolia; } 40+\text { synonyms }\end{array}$ \\
\hline & & Brno, 1963 & $\begin{array}{l}\text { no further } \\
\text { record }\end{array}$ & no & \\
\hline & & nn, 1972 & $\begin{array}{l}1980 \\
\text { ("incorrect!") }\end{array}$ & no & \\
\hline & & Kaunas, 1970 & 1971 & no & \\
\hline & $\begin{array}{l}\text { Peperomia marmorata } \\
\text { Hook. f. }\end{array}$ & $\begin{array}{l}\text { Amsterdam, } \\
1961\end{array}$ & $\begin{array}{l}\text { no further } \\
\text { record }\end{array}$ & no & \\
\hline
\end{tabular}




\begin{tabular}{|c|c|c|c|c|c|}
\hline & & \begin{tabular}{|l} 
Cluj-Napoca, \\
$1964 \& 1966$ \\
\end{tabular} & $1966 \& 1969$ & no & \\
\hline & & Košice, 1970 & 1975 & no & \\
\hline & & Caen, 1981 & 2005 (2007) & no & \\
\hline & $\begin{array}{l}\text { Peperomia metallica } \\
\text { Linden \& Rodigas }\end{array}$ & $\begin{array}{l}\text { Cluj-Napoca, } \\
1966\end{array}$ & 1981 & no & \\
\hline & & $\begin{array}{l}\text { Caen, } 1981 \& \\
1988\end{array}$ & $\begin{array}{l}1984(1989) \& \\
2003(2010)\end{array}$ & no & \\
\hline & $\begin{array}{l}\text { Peperomia obtusifolia } \\
\text { (L.) A. Dietr. }\end{array}$ & $\begin{array}{l}\text { Cluj-Napoca, } \\
1966\end{array}$ & 1991 & no & $40+$ synonyms \\
\hline & & Vacratot, 1961 & $1961(1975)$ & no & \\
\hline & & Genova, 1963 & $1963(1975)$ & no & \\
\hline & $\begin{array}{l}\text { Peperomia obtusifolia } \\
\text { (L.) A. Dietr. f. } \\
\text { magnoliaefolia (?) }\end{array}$ & Kaunas, 1961 & 1981 (1989) & no & $\begin{array}{l}\text { 40+ species-synonyms; } \\
\text { unknown form }\end{array}$ \\
\hline & $\begin{array}{l}\text { Peperomia pellucida } \\
\text { (L.) Kunth }\end{array}$ & Brno, 1989 & 1989 & no & $10+$ synonyms \\
\hline & $\begin{array}{l}\text { Peperomia pereskiifolia } \\
\text { (Jacq.) Kunth }\end{array}$ & nn, 1963 & 1972 (1975) & no & 10 synonyms \\
\hline & $\begin{array}{l}\text { Peperomia } \\
\text { puberulispica C. DC. }\end{array}$ & $\begin{array}{l}\text { Amsterdam, } \\
1971\end{array}$ & 1972 (1975) & no & \\
\hline & $\begin{array}{l}\text { Peperomia reptilis } \mathrm{C} . \\
\text { DC. }\end{array}$ & Besancon, 1992 & $\begin{array}{l}\text { no further } \\
\text { record }\end{array}$ & no & \\
\hline & $\begin{array}{l}\text { Peperomia rotundifolia } \\
\text { (L.) Kunth }\end{array}$ & Duisburg, 1986 & 1988 & no & $\begin{array}{l}\text { arrived as P.nummularifolia, } \\
\text { one of } 20+\text { synonyms }\end{array}$ \\
\hline & & $\begin{array}{l}\text { Antwerp, } 1960 \& \\
1966\end{array}$ & $1961 \& 1972$ & no & $20+$ synonyms \\
\hline & & Naples, 1963 & 1969 & no & \\
\hline & & Vacratot, 1965 & $1973(1975)$ & no & \\
\hline & $\begin{array}{l}\text { Peperomia serpens } \\
\text { (Sw.) Loudon }\end{array}$ & nn, 1961 & 1977 & no & $\begin{array}{l}\text { arrived as P.scandens, one } \\
\text { of } 30+\text { synonyms }\end{array}$ \\
\hline & $\begin{array}{l}\text { Peperomia subpeltata } \\
\text { (Willd.) A. Dietr. }\end{array}$ & Gent, 1979 & $\begin{array}{l}1980 \\
\text { ("incorrect!") }\end{array}$ & no & det.as P. sarcophylla \\
\hline & $\begin{array}{l}\text { Peperomia subrenifolia } \\
\text { Trel. \& Yunck. }\end{array}$ & Nantes, 1989 & $2003(2010)$ & no & $\begin{array}{l}\text { accepted name is also } P \text {. } \\
\text { quadrifolia; } 20+\text { synonyms, } \\
\text { among which is Piper } \\
\text { quadrifolium "!" }\end{array}$ \\
\hline & $\begin{array}{l}\text { Peperomia theodori } \\
\text { Trel. }\end{array}$ & Goeteborg, 1970 & 1975 & no & syn. $P$. reflexa f. longilimba \\
\hline & $\begin{array}{l}\text { Peperomia trinervis } \\
\text { Ruiz \& Pav. }\end{array}$ & Duisburg, 1982 & 1985 (1989) & no & 3 synonyms \\
\hline & $\begin{array}{l}\text { Peperomia velutina } \\
\text { Linden \& André }\end{array}$ & $\begin{array}{l}\text { Cantonspark } \\
\text { (Baarn), } 1953\end{array}$ & 1963 & no & \\
\hline & & Gent, 1979 & $2003(2010)$ & no & \\
\hline & & Nancy, 1981 & 1991 & no & \\
\hline & $\begin{array}{l}\text { Peperomia } \\
\text { verschaffeltii Lem. }\end{array}$ & Duisburg, 1986 & 1991 & no & 2 synonyms \\
\hline & & Caen, 1988 & 2007 & no & \\
\hline & $\begin{array}{l}\text { Peperomia verticillata } \\
\text { (L.) A. Dietr. }\end{array}$ & Krakow, 1961 & $\begin{array}{l}\text { no further } \\
\text { record }\end{array}$ & no & 3 synonyms \\
\hline & & Vienna, 1961 & 1977 & no & \\
\hline & Piper betle L. & Besancon, 1965 & 1989 & no & \\
\hline
\end{tabular}




\begin{tabular}{|c|c|c|c|c|c|}
\hline & & Dijon, 1986 & 1989 & no & \\
\hline & $\begin{array}{l}\text { Piper officinarum } \\
\text { (Miq.) C. DC. }\end{array}$ & Muenster, 1967 & 1968 & no & $\begin{array}{l}\text { accepted name is also } \\
\text { P.retrofractum; } 3 \text { synonyms }\end{array}$ \\
\hline & $\begin{array}{l}\text { Piper ornatum N.E. } \\
\text { Br. }\end{array}$ & Vacratot, 1967 & $1978(1989)$ & no & \\
\hline \multirow[t]{5}{*}{ Rubiaceae } & $\begin{array}{l}\text { Gardenia jasminoides } \\
\text { J. Ellis var. } \\
\text { jasminoides }\end{array}$ & Kyoto, 1980 & 1984 & no & $\begin{array}{l}3 \text { species-synonyms; } \\
\text { arrived as G. jasminoides } \mathrm{f} . \\
\text { grandiflora, one of } 20 \\
\text { synonyms }\end{array}$ \\
\hline & Hamelia patens Jacq. & $\begin{array}{l}\text { Bochum, } 1980 \& \\
1981\end{array}$ & 1984 both & no & $15+$ synonyms \\
\hline & $\begin{array}{l}\text { Hydnophytum } \\
\text { formicarum Jack }\end{array}$ & $\begin{array}{l}\text { Duesseldorf, } \\
2004\end{array}$ & 2005 & no & 1 synonym \\
\hline & $\begin{array}{l}\text { Nertera granadensis } \\
\text { (Mutis ex L. f.) Druce }\end{array}$ & nn, 1970 & 1971 & no & $\begin{array}{l}2 \text { more accepted names; } 5+ \\
\text { synonyms }\end{array}$ \\
\hline & Paederia foetida $\mathrm{L}$. & Lushan, 1987 & 1987 & no & $\begin{array}{l}\text { arrived as } P \text {. scandens; } \\
\text { accepted name is also } P \text {. } \\
\text { diffusa; } 15+\text { synonyms }\end{array}$ \\
\hline \multirow[t]{4}{*}{ Rutaceae } & \begin{tabular}{|l|} 
Erythrochiton \\
brasiliensis Nees \& C. \\
Mart.
\end{tabular} & $\begin{array}{l}\text { Vienna- } \\
\text { Belvedere, } 1956\end{array}$ & 1961 & no & $\begin{array}{l}\text { accepted name is also } \\
\text { Pentamorpha graveolens }\end{array}$ \\
\hline & & $\begin{array}{l}\text { Frankfurt, } 1963 \\
\& 1968\end{array}$ & $1965 \& 1969$ & no & \\
\hline & & Bern, 1966 & 1969 & no & \\
\hline & $\begin{array}{l}\text { Severinia buxifolia } \\
\text { (Poir.) Ten. }\end{array}$ & Havana, 1990 & 1996 & no & \\
\hline Sapindaceae & $\begin{array}{l}\text { Sapindus laurifolia } \\
\text { Vahl. }\end{array}$ & Annoville, 1990 & 1991 & no & $\begin{array}{l}\text { acc.to IPNI; in Tropicos } \\
\text { and Plant List (S. } \\
\text { laurifolius) it is an } \\
\text { unresolved name }\end{array}$ \\
\hline Saxifragaceae & $\begin{array}{l}\text { Rodgersia podophylla } \\
\text { A. Gray }\end{array}$ & Nasu, 2004 & 2005 & yes & relocated, syn. R. japonica \\
\hline \multirow[t]{4}{*}{ Solanaceae } & $\begin{array}{l}\text { Browallia speciosa } \\
\text { Hook. }\end{array}$ & nn, 1974 & 1975 & no & \\
\hline & $\begin{array}{l}\text { Cestrum roseum } \\
\text { Kunth }\end{array}$ & nn, 1963 & 1972 (1975) & no & syn. Habrothamnus roseus \\
\hline & $\begin{array}{l}\text { Jaborosa integrifolia } \\
\text { Lam. }\end{array}$ & Bologna, 1968 & 1968 & no & syn. J. bonariensis \\
\hline & $\begin{array}{l}\text { Vestia foetida } \\
\text { Hoffmanns. }\end{array}$ & Chelsea, 2003 & 2009 (2010) & no & monotypic; syn. $V$. lycioides \\
\hline \multirow[t]{5}{*}{ Urticaceae } & $\begin{array}{l}\text { Pilea microphylla (L.) } \\
\text { Liebm. }\end{array}$ & Coimbra, 1961 & 1963 & no & $10+$ synonyms \\
\hline & & Amsterdan, 1987 & 2010 & no & \\
\hline & $\begin{array}{l}\text { Pilea mollis Wedd. } \\
\text { ‘Moon Valley' }\end{array}$ & Vienna, 1971 & 1984 & no & \\
\hline & $\begin{array}{l}\text { Pilea pumila (L.) A. } \\
\text { Gray }\end{array}$ & Goettingen, 1989 & $\begin{array}{l}\text { no further } \\
\text { record }\end{array}$ & no & syn. Urtica pumila \\
\hline & Pilea spruceana Wedd. & London, 1959 & 1979 & no & \\
\hline Verbenaceae & Lantana involucrata L. & Bonn, 1981 & 1984 & no & 4 synonyms \\
\hline \multirow[t]{2}{*}{ Vitaceae } & Cissus sp. & Prague, 1964 & 1983 & no & \\
\hline & $\begin{array}{l}\text { Cissus njegerre Gilg \& } \\
\text { H.C. Strauss }\end{array}$ & Giessen, 1988 & 2012 (2013) & no & \\
\hline Winteraceae & $\begin{array}{l}\text { Tasmannia lanceolata } \\
\text { (Poir.) A.C.Sm. }\end{array}$ & nn, 2008 & 2010 & no & \\
\hline
\end{tabular}




\begin{tabular}{|c|c|c|c|c|c|}
\hline & $\begin{array}{l}\text { MONOCOTYLE- } \\
\text { DONS (Liliopsida) }\end{array}$ & & & & \\
\hline \multirow[t]{15}{*}{ Amaryllidaceae } & $\begin{array}{l}\text { Haemanthus } \\
\text { magnificus Herb. }\end{array}$ & $\begin{array}{l}\text { Besancon, } 1969 \\
\& 1971\end{array}$ & 1979 (both) & no & $\begin{array}{l}\text { accepted name is also } \\
\text { Scadoxus puniceus }\end{array}$ \\
\hline & $\begin{array}{l}\text { Hippeastrum aulicum } \\
\text { Herb. }\end{array}$ & $\begin{array}{l}\text { Amsterdam, } \\
1957\end{array}$ & 1968 & no & syn. Amaryllis aulica \\
\hline & & Potsdam, 1978 & 1979 & no & \\
\hline & $\begin{array}{l}\text { Hippeastrum } \\
\text { brachyandrum Baker }\end{array}$ & Koeln, 1959 & 1981 & no & $\begin{array}{l}\text { accepted name is also } \\
\text { Habranthus brachyandrus; } 2 \\
\text { synonyms }\end{array}$ \\
\hline & & Groningen, 1964 & 1968 & no & \\
\hline & & Basel, 1975 & 1988 & no & \\
\hline & & Haren, 1988 & $\begin{array}{l}\text { no further } \\
\text { record }\end{array}$ & no & \\
\hline & $\begin{array}{l}\text { Hippeastrum } \\
\text { reticulatum Herb.* }\end{array}$ & Louvain, 1961 & $\begin{array}{l}1979 \\
\text { ("incorrect!") }\end{array}$ & no & det. as Zephyranthes sp. \\
\hline & $\begin{array}{l}\text { Hippeastrum rutilum } \\
\text { (Ker Gawl.) Herb. } \\
\text { var. fulgidum } \\
\text { Sprenger }\end{array}$ & $\begin{array}{l}\text { Vienna, 1965, } \\
1967,1968,1969, \\
1976\end{array}$ & $\begin{array}{l}1981,1975 \\
1972,1972 \\
2000\end{array}$ & no & $\begin{array}{l}2 \text { more accepted species- } \\
\text { names; syn. Amaryllis } \\
\text { fulgida }\end{array}$ \\
\hline & $\begin{array}{l}\text { Hymenocallis rotata } \\
\text { (Ker Gawl.) Herb. }\end{array}$ & Zuerich, 1960 & 1963 & no & 4 synonyms \\
\hline & $\begin{array}{l}\text { Ipheion uniflorum } \\
\text { (Lindl.) Raf. }\end{array}$ & Basel, 1966 & 1980 & no & $\begin{array}{l}\text { accepted name is also } \\
\text { Tristagma uniflorum; } 8 \\
\text { synonyms }\end{array}$ \\
\hline & \begin{tabular}{|l|} 
Scadoxus multiflorus \\
(Martyn) Raf. subsp. \\
katharinae Friis \& \\
Nordal \\
\end{tabular} & nn, 2000 & 2000 & no & $\begin{array}{l}\text { arrived as Haemanthus } \\
\text { katharinae, a single valid } \\
\text { synonym }\end{array}$ \\
\hline & & nn, 1961 & 2000 & no & \\
\hline & & Essen, 1969 & 1971 & no & \\
\hline & & Wroclaw, 1969 & 1973 & no & \\
\hline \multirow[t]{9}{*}{ Araceae } & $\begin{array}{l}\text { Aglaonema costatum } \\
\text { N.E. Br. }\end{array}$ & Duisburg, 1960 & 1961 & no & 2 synonyms \\
\hline & $\begin{array}{l}\text { Aglaonema marantifo- } \\
\text { lium Blume }\end{array}$ & $\begin{array}{l}\text { Pavia 1967, } 1969 \\
\& 1970\end{array}$ & $\begin{array}{l}1972,1972 \& \& \\
1971\end{array}$ & no & $\begin{array}{l}\text { arrived as Scindapsus } \\
\text { erectus, one of } 5 \text { synonyms }\end{array}$ \\
\hline & & Dresden, 1967 & $1972(1975)$ & no & $\begin{array}{l}\text { arrived as A.oblongifolium, } \\
\text { invalid synonym among } \\
\text { others }\end{array}$ \\
\hline & $\begin{array}{l}\text { Aglaonema marmora- } \\
\text { tum Engl. }\end{array}$ & Besancon, 1965 & 1968 & no & $\begin{array}{l}\text { accepted name is also } \\
\text { A.pumilum }\end{array}$ \\
\hline & & Greifswald, 1966 & 1978 & no & \\
\hline & $\begin{array}{l}\text { Alocasia indica (Lour.) } \\
\text { Spach }\end{array}$ & Besancon, 1964 & 1966 & no & $\begin{array}{l}\text { arrived as Arum indicum, } \\
\text { one of } 40+\text { synonyms; } \\
\text { accepted name is also } \\
\text { A.macrorrhizos }\end{array}$ \\
\hline & & nn, 1961 & $\begin{array}{l}\text { no further } \\
\text { record }\end{array}$ & no & $\begin{array}{l}\text { arrived as Colocasia indica, } \\
\text { accepted name as well as } \\
\text { A.macrorrhizos }\end{array}$ \\
\hline & $\begin{array}{l}\text { Alocasia korthalsii } \\
\text { Schott }\end{array}$ & nn, 1961 & $\begin{array}{l}\text { no further } \\
\text { record }\end{array}$ & no & syn. A.thibautiana \\
\hline & $\begin{array}{l}\text { Alocasia sanderiana W. } \\
\text { Bull }\end{array}$ & Goeteborg, 1978 & 1988 & no & \\
\hline
\end{tabular}




\begin{tabular}{|c|c|c|c|c|c|}
\hline & $\begin{array}{l}\text { Arisaema consangui- } \\
\text { neum Schott }\end{array}$ & Turku, 1985 & 1988 & no & $\begin{array}{l}\text { accepted name is also } A \text {. } \\
\text { erubescens; } 4 \text { synonyms }\end{array}$ \\
\hline & $\begin{array}{l}\text { Arisaema ringens } \\
\text { (Thunb.) Schott var. } \\
\text { praecox (de Vriese ex } \\
\text { K. Koch) Engl. }\end{array}$ & Basel, 1970 & 1972 & no & $\begin{array}{l}\text { accepted name is also } A \text {. } \\
\text { reingens; there is also a " } \mathrm{f} \text {. } \\
\text { praecox" }\end{array}$ \\
\hline & $\begin{array}{l}\text { Caladium bicolor } \\
\text { (Aiton) Vent. }\end{array}$ & Bergen, 1973 & 1975 & no & $\begin{array}{l}\text { arrived as C. hortulanum; } \\
\text { accepted name is C. } \mathrm{x} \\
\text { hortulanum }\end{array}$ \\
\hline & & $\begin{array}{l}\text { Linz, } 1955 \& \\
1963\end{array}$ & $1961 \& 1966$ & no & $\begin{array}{l}\text { arrived as Arum bicolor, one } \\
\text { of } 50+\text { synonyms }\end{array}$ \\
\hline & & Bratislava, 1966 & 1970 & no & $50+$ synonyms \\
\hline & & $\begin{array}{l}\text { Cluj-Napoca, } \\
1966 \text { \& } 1967 \\
\end{array}$ & 1977 both & no & \\
\hline & & Potsdam, 1968 & $\begin{array}{l}\text { no further } \\
\text { record }\end{array}$ & no & \\
\hline & & $\begin{array}{l}\text { Oslo, } 1968 \& \\
1969\end{array}$ & 1975 both & no & \\
\hline & $\begin{array}{l}\text { Dieffenbachia } \\
\text { "japonica viridis" (?) }\end{array}$ & $\begin{array}{l}\text { "Dalmatia", } 1961 \\
\& 1975\end{array}$ & $\begin{array}{l}\text { no further } \\
\text { records }\end{array}$ & no & $\begin{array}{l}\text { unknown taxon and origin: } \\
\text { probably a gift }\end{array}$ \\
\hline & $\begin{array}{l}\text { Epipremnum } \\
\text { pinnatum (L.) Engl. }\end{array}$ & Naples, 1963 & 1968 & no & $\begin{array}{l}\text { arrived as E. mirabile, one } \\
\text { of } 40+\text { synonyms }\end{array}$ \\
\hline & Pothos "platycaule" (?) & Nancy, 1963 & 1978 & no & $\begin{array}{l}\text { unknown species - maybe } \\
\text { as a counterpart of } P \text {. } \\
\text { "acaule"? }\end{array}$ \\
\hline & $\begin{array}{l}\text { Rhaphidophora } \\
\text { africana N.E. Br. }\end{array}$ & Besancon, 1972 & 1975 & no & \\
\hline & $\begin{array}{l}\text { Rhaphidophora } \\
\text { decursiva (Roxb.) } \\
\text { Schott }\end{array}$ & $\begin{array}{l}\text { Antwerp, } 1965 \& \\
1970\end{array}$ & $\begin{array}{l}1966 \& 1971 \\
(1975)\end{array}$ & no & 8 synonyms \\
\hline & $\begin{array}{l}\text { Sauromatum venosum } \\
\text { (Aiton) Kunth }\end{array}$ & Halle, 2001 & $2004(2010)$ & yes & $\begin{array}{l}\text { accepted name is also } \\
\text { Typhonium venosum; 30+ } \\
\text { synonyms, ima } 12767 \\
\text { Dusseldorf } 05 \\
\end{array}$ \\
\hline & \begin{tabular}{|l|} 
Typhonodorum \\
lindleyanum Schott
\end{tabular} & Muenster, 1967 & 1968 & no & monotypic; 2 synonyms \\
\hline & $\begin{array}{l}\text { Xanthosoma violaceum } \\
\text { Schott }\end{array}$ & Kiel, 1966 & 1968 & no & $\begin{array}{l}2 \text { more accepted names; } 2 \\
\text { synonyms }\end{array}$ \\
\hline & & Besancon, 1968 & 1968 & no & \\
\hline & & Oslo, 1968 & $\begin{array}{l}\text { no further } \\
\text { record }\end{array}$ & no & \\
\hline & \begin{tabular}{|l|} 
Zantedeschia \\
aethiopica (L.) Spreng.
\end{tabular} & Palermo, 1969 & 2013 & yes & relocated; 4 synonyms \\
\hline & $\begin{array}{l}\text { Zantedeschia } \\
\text { rehmannii Engl. }\end{array}$ & Stockholm, 1968 & 1972 & yes & \\
\hline & & Wroclaw, 1986 & 1988 & yes & \\
\hline & & Bogor, 2004 & 2005 & yes & \\
\hline \multirow[t]{3}{*}{ Asparagaceae } & $\begin{array}{l}\text { Asparagus macowanii } \\
\text { Baker 'Blossfeld' }\end{array}$ & nn, 1985 & \begin{tabular}{|l}
2005 \\
(relocated)
\end{tabular} & yes & \\
\hline & $\begin{array}{l}\text { Asparagus setaceus } \\
\text { (Kunth) Jessop } \\
\text { 'Cupressoides' } \\
\end{array}$ & Warsaw, 1986 & $1991(1996)$ & no & $\begin{array}{l}\text { accepted species name is } \\
\text { also Protasparagus s.; } 4 \\
\text { synonyms }\end{array}$ \\
\hline & $\begin{array}{l}\text { Asparagus sprengeri } \\
\text { Regel }\end{array}$ & nn, 1963 & $1989(1996)$ & no & $\begin{array}{l}\text { accepted names are also } \\
\text { A.densiflorus and } \\
\text { A.aethiopicus }\end{array}$ \\
\hline
\end{tabular}




\begin{tabular}{|c|c|c|c|c|c|}
\hline & $\begin{array}{l}\text { Aspidistra elatior } \\
\text { Blume 'Variegata' }\end{array}$ & nn, $1961 \& 1981$ & $\begin{array}{l}1989 \& 2002 \\
(2005)\end{array}$ & no & 2 species-synonyms \\
\hline & $\begin{array}{l}\text { Chlorophytum capense } \\
\text { (L.) Voss 'Variegatum' }\end{array}$ & Debrecen, 1968 & 1968 & no & 4 species-synonyms \\
\hline & $\begin{array}{l}\text { Chlorophytum } \\
\text { inornatum Ker Gawl. }\end{array}$ & Berlin, 1973 & 1991 & no & \\
\hline & $\begin{array}{l}\text { Chlorophytum } \\
\text { madagascariense Baker }\end{array}$ & Bergen, 2004 & 2005 & yes & $\begin{array}{l}\text { accepted synonym is also } \\
\text { Ch.gramineum; } 3 \text { synonyms }\end{array}$ \\
\hline & & Karlsruhe, 1965 & 1966 & yes & \\
\hline & & Bogor, 2004 & 2005 & yes & \\
\hline & & Innsbruck, 2004 & 2005 & yes & \\
\hline & $\begin{array}{l}\text { Cordyline australis } \\
\text { Hook. f. } \\
\text { 'Atropurpurea' }\end{array}$ & Adelaide, 1971 & 1989 & yes & \\
\hline & $\begin{array}{l}\text { Cordyline indivisa (G. } \\
\text { Forst.) Endl. }\end{array}$ & Invercargill, 1986 & 1988 & no & 3 homonyms, 2 still valid \\
\hline & & Wuppertal, 1990 & 1991 & no & \\
\hline & $\begin{array}{l}\text { Cordyline terminalis } \\
\text { (L.) Kunth 'Baueri' }\end{array}$ & Norfolk, 1981 & 1989 & no & $\begin{array}{l}\text { arrived as C. baueri } \\
\text { - probably a synonim of } \\
\text { C.t. 'B.' }\end{array}$ \\
\hline & & Zuerich, 1959 & 1979 & no & \\
\hline & $\begin{array}{l}\text { Cordyline terminalis } \\
\text { (L.) Kunth 'Youngii' }\end{array}$ & Kiel, 1972 & 1978 & no & $\begin{array}{l}\text { arrived as C. terminalis } \\
\text { 'Youngi' }\end{array}$ \\
\hline & $\begin{array}{l}\text { Dracaena fragrans (L.) } \\
\text { Ker Gawl. } \\
\text { 'Compacta' }\end{array}$ & nn, 2003 & 2010 & no & $\begin{array}{l}\text { purchased; } 3 \text { species } \\
\text { synonyms, incl. } D . \\
\text { deremensis }\end{array}$ \\
\hline & $\begin{array}{l}\text { Dracaena fragrans (L.) } \\
\text { Ker-Gawl. } \\
\text { 'Massangeana' } \\
\end{array}$ & nn, 2003 & 2008 & no & $\begin{array}{l}\text { purchased; } 3 \text { species } \\
\text { synonyms, incl. } D . \\
\text { deremensis }\end{array}$ \\
\hline & $\begin{array}{l}\text { Dracaena godseffiana } \\
\text { Sander ex Mast. }\end{array}$ & $\begin{array}{l}\text { "Lada" Nursery } \\
\text { Slovenia, } 1964\end{array}$ & $\begin{array}{l}\text { no further } \\
\text { record }\end{array}$ & no & $\begin{array}{l}\text { purchased; several } \\
\text { synonyms }\end{array}$ \\
\hline & & Duisburg, 1969 & 1977 & no & several synonyms \\
\hline & Dracaena 'Haageana' & Palermo, 1948 & 1977 & no & before WWII; hort. \\
\hline & $\begin{array}{l}\text { Dracaena hookeriana } \\
\text { K. Koch }\end{array}$ & nn, $1961 \& 1973$ & $\begin{array}{l}\text { no further } \\
\text { records }\end{array}$ & no & syn. D.transvaalensis \\
\hline & $\begin{array}{l}\text { Dracaena sanderiana } \\
\text { Sander }\end{array}$ & $\begin{array}{l}\text { "Lada" Nursery } \\
\text { Slovenia, } 1964\end{array}$ & 1966 (1989) & no & $\begin{array}{l}\text { homonym D.sanderiana } \\
\text { hort. }\end{array}$ \\
\hline & $\begin{array}{l}\text { Dracaena surculosa } \\
\text { Lindl. var. maculata } \\
\text { Hook. f. 'Florida } \\
\text { Beauty' } \\
\end{array}$ & Turku, 1990 & 1991 & no & \\
\hline & Eucomis bicolor Baker & Uppsala, 1957 & 2013 & no & \\
\hline & $\begin{array}{l}\text { Lachenalia reflexa } \\
\text { Thunb. }\end{array}$ & Kyoto, 2002 & 2010 & no & relocated \\
\hline & $\begin{array}{l}\text { Nolina greenei S. } \\
\text { Watson ex Trel. }\end{array}$ & nn, 1990 & $2005(2010)$ & no & \\
\hline & $\begin{array}{l}\text { Nolina longifolia } \\
\text { (Karw. ex Schult. f.) } \\
\text { Hemsl. }\end{array}$ & $\begin{array}{l}\text { Berlin-Dahlem, } \\
1951\end{array}$ & 2010 & no & $\begin{array}{l}\text { relocated; } 3 \text { synonyms, } \\
\text { incl. Beaucarnea } l .\end{array}$ \\
\hline & $\begin{array}{l}\text { Nolina matapensis } \\
\text { Wiggins }\end{array}$ & San Marino, 1976 & 2010 & yes & relocated \\
\hline & $\begin{array}{l}\text { Nolina microcarpa S. } \\
\text { Watson }\end{array}$ & Barcelona, 1985 & $2005(2010)$ & no & 2 synonyms \\
\hline
\end{tabular}




\begin{tabular}{|c|c|c|c|c|c|}
\hline & $\begin{array}{l}\text { Nolina stricta (Lem.) } \\
\text { Cif. \& Giacom. }\end{array}$ & Adelaide, 1953 & 1991 (1995) & no & $\begin{array}{l}\text { accepted name is also } \\
\text { Beaucarnea stricta; } 5 \\
\text { synonyms }\end{array}$ \\
\hline & $\begin{array}{l}\text { Sansevieria dooneri } \\
\text { N.E. Br. }\end{array}$ & nn, 2003 & 2005 & no & \\
\hline & $\begin{array}{l}\text { Sansevieria ehrenbergi } \\
\text { Schweinf. ex Baker* }\end{array}$ & $\begin{array}{l}\text { Bucharest, } 1971 \\
\& 1972\end{array}$ & $\begin{array}{l}1980 \text { \& } 1981 \\
\text { ("incorrect!") }\end{array}$ & no & \\
\hline & \begin{tabular}{|l|} 
Sansevieria grandis \\
Hook. f.
\end{tabular} & nn, 1961 & 1989 & no & \\
\hline & $\begin{array}{l}\text { Sansevieria liberica } \\
\text { Gérôme \& Labroy }\end{array}$ & Legon, 1967 & $1969(1975)$ & no & \\
\hline & $\begin{array}{l}\text { Sansevieria parva N.E. } \\
\text { Br. }\end{array}$ & Goeteborg, 1978 & 2007 & no & \\
\hline & $\begin{array}{l}\text { Veltheimia bracteata } \\
\text { Harv. ex Baker }\end{array}$ & Rotterdam, 1969 & 2010 & no & arrived as syn. $V$. viridifolia \\
\hline & Veltheimia capensis DC. & Vienna, 2012 & 2013 & no & 3 synonyms \\
\hline & Veltheimia glauca Jacq. & nn, $1961 \& 1971$ & $\begin{array}{l}1968 \& 1984 \\
(1989)\end{array}$ & no & $\begin{array}{l}\text { accepted name is also } V \text {. } \\
\text { capensis }\end{array}$ \\
\hline & $\begin{array}{l}\text { Yucca aloifolia L. } \\
\text { 'Variegata'* }\end{array}$ & Monaco, 1994 & $\begin{array}{l}2000 \\
(\text { "incorrect!") }\end{array}$ & yes & 3 species-synonyms \\
\hline \multirow[t]{16}{*}{ Bromeliaceae } & $\begin{array}{l}\text { Aechmea candida } \mathrm{E} . \\
\text { Morren ex Baker }\end{array}$ & $\begin{array}{l}\text { nn, in collection } \\
\text { in } 2000\end{array}$ & $2010(2011)$ & no & 1 synonym \\
\hline & $\begin{array}{l}\text { Aechmea cariocae L.B. } \\
\text { Sm }\end{array}$ & $\begin{array}{l}\text { Bruxelles, } 1965 \& \\
1969\end{array}$ & $1995 \& 1977$ & no & \\
\hline & $\begin{array}{l}\text { Aechmea mexicana } \\
\text { Baker }\end{array}$ & Muenchen, 1986 & 2006 (2007) & no & 7 synonyms \\
\hline & $\begin{array}{l}\text { Aechmea mexicana } \\
\text { Baker var. nudicaulis } \\
(?)\end{array}$ & Bruxelles, 1965 & 1969 & no & $\begin{array}{l}8 \text { synonyms; unknown } \\
\text { variety (perhaps } A e . \\
\text { nudicaulis?) }\end{array}$ \\
\hline & $\begin{array}{l}\text { Aechmea nudicaulis } \\
\text { (L.) Griseb. }\end{array}$ & nn, 2004 & 2008 & no & $20+$ synonyms \\
\hline & $\begin{array}{l}\text { Aechmea nudicaulis } \\
\text { (L.) Griseb. var. } \\
\text { nudicaulis }\end{array}$ & Utrecht, 2004 & 2005 & no & $10+$ synonyms \\
\hline & $\begin{array}{l}\text { Aechmea racinae L.B. } \\
\text { Sm }\end{array}$ & Prague, 1964 & 1978 & no & \\
\hline & $\begin{array}{l}\text { Aechmea recurvata } \\
\text { (Klotzsch) L.B. Sm. } \\
\text { var. ortgiesii (Baker) } \\
\text { Reitz }\end{array}$ & Prague, 1964 & 1984 & no & $\begin{array}{l}4 \text { synonyms; accepted } \\
\text { name is also Ae.r. var. } \\
\text { albobracteata }\end{array}$ \\
\hline & & Essen, 1968 & 1972 & no & \\
\hline & $\begin{array}{l}\text { Ananas comosus (L.) } \\
\text { Merr. }\end{array}$ & nn, 1956 \& 1966 & $1963 \& 1978$ & no & 13 synonyms \\
\hline & $\begin{array}{l}\text { Araeococcus } \\
\text { goeldianus L.B. Sm. }\end{array}$ & $\begin{array}{l}\text { nn, in collection } \\
\text { in } 2000\end{array}$ & 2005 (2006) & no & \\
\hline & $\begin{array}{l}\text { Billbergia decora } \\
\text { Poepp. \& Endl. }\end{array}$ & Erlangen, 1965 & 1969 & no & \\
\hline & & Gent, 1965 & 1995 & no & \\
\hline & $\begin{array}{l}\text { Billbergia viridiflora } \\
\text { H.L. Wendl. }\end{array}$ & Utrecht, 2004 & 2005 (2006) & no & 3 synonyms \\
\hline & Bromelia balansae $\mathrm{Mez}$ & nn, 1953 & 1980 & no & $\begin{array}{l}\text { arrived as B. pinguin, one } \\
\text { of } 4 \text { synonyms }\end{array}$ \\
\hline & \begin{tabular}{|l|} 
Bromelia nidus-puellae \\
(André) André ex \\
Mez $^{*}$
\end{tabular} & Gent, 1955 & $\begin{array}{l}2010 \\
(\text { "incorrect!") }\end{array}$ & no & $\begin{array}{l}\text { arrived as "Karatas puellae" } \\
\text { (unknown member of fam. } \\
\text { Bromeliaceaea) }\end{array}$ \\
\hline
\end{tabular}




\begin{tabular}{|c|c|c|c|c|c|}
\hline & $\begin{array}{l}\text { Canistrum lindenii } \\
\text { (Regel) Mez }\end{array}$ & “Erute”, 1967 & 1978 & no & $\begin{array}{l}\text { accepted name is also } \\
\text { Edmundoa lindenii var. rosea; } \\
12 \text { synonyms }\end{array}$ \\
\hline & $\begin{array}{l}\text { Cryptanthus acaulis } \\
\text { (Lindl.) Beer }\end{array}$ & Duisburg, 1961 & 1975 & no & \\
\hline & $\begin{array}{l}\text { Cryptanthus acaulis } \\
\text { (Lindl.) Beer var. } \\
\text { ruber hort. ex Beer }\end{array}$ & Duisburg, 1961 & 1972 & no & \\
\hline & $\begin{array}{l}\text { Cryptanthus } \\
\text { 'Aurantiacus' }\end{array}$ & nn, 1963 & 1968 & no & arrived as "C. aurantiaca" \\
\hline & Dyckia brevifolia Baker & Barcelona, 1981 & 1988 & no & 3 synonyms \\
\hline & $\begin{array}{l}\text { Fosterella penduliflora } \\
\text { (C.H. Wright) L.B. } \\
\text { Sm. }\end{array}$ & Berlin, 1985 & 1986 & no & 4 synonyms \\
\hline & & Trondheim, 1989 & $1991(1995)$ & no & \\
\hline & & Warsaw, 1989 & $1991(1995)$ & no & \\
\hline & $\begin{array}{l}\text { Guzmania fastuosa } \\
\text { (André) André ex } \\
\text { Mez }\end{array}$ & $\begin{array}{l}\text { Leningrad (St. } \\
\text { Petersburg), } 1958\end{array}$ & 1968 & no & $\begin{array}{l}2 \text { more accepted names; } \\
10+\text { synonyms }\end{array}$ \\
\hline & $\begin{array}{l}\text { Karatas nidus-puellae } \\
\text { Andrè }\end{array}$ & Gent, 1955 & $1984(1988)$ & no & $\begin{array}{l}\text { accepted name is also } \\
\text { Bromelia nidus-puellae }\end{array}$ \\
\hline & $\begin{array}{l}\text { Neoregelia spectabilis } \\
\text { (Moore) L.B. Sm. }\end{array}$ & $\begin{array}{l}\text { Prague, } 1964 \& \\
1967\end{array}$ & 1995 \& 1975 & no & 5 synonyms \\
\hline & Nidularium 'Tricolor' & $\begin{array}{l}\text { "Lada" Nursery } \\
\text { Slovenia, } 1964\end{array}$ & $1966(1975)$ & no & arrived as "N. tricolor" \\
\hline & $\begin{array}{l}\text { Pitcairnia albucifolia } \\
\text { Schrad.* }\end{array}$ & nn, 1963 & $\begin{array}{l}\text { 1981, } \\
\text { "incorrect" }\end{array}$ & no & $\begin{array}{l}\text { accepted name is also } \\
\text { Pitcairnia spicata; } 10+ \\
\text { synonyms }\end{array}$ \\
\hline & $\begin{array}{l}\text { Pitcairnia andreana } \\
\text { Linden }\end{array}$ & Vienna, 1965 & 1969 & no & 3 synonyms \\
\hline & $\begin{array}{l}\text { Pitcairnia } \\
\text { aphelandriflora Lem. }\end{array}$ & Brisel, 1966 & 1968 & no & 3 synonyms \\
\hline & Puya coerulea Lindl. & Berkeley, 1961 & $\begin{array}{l}\text { no further } \\
\text { record }\end{array}$ & no & 3 synonyms \\
\hline & Puya gigas André & Košice, 1965 & 1980 & no & $\begin{array}{l}\text { CR B1ab(iii) - Critically } \\
\text { Endangered - Global }\end{array}$ \\
\hline & $\begin{array}{l}\text { Puya mirabilis (Mez) } \\
\text { L.B. Sm. }\end{array}$ & Nancy, 1981 & 1991 & no & 2 synonyms \\
\hline & & Nantes, 1986 & 1993 & no & \\
\hline & Puya raimondii Harms & Berkeley, 1988 & 1991 (1995) & no & syn. Pourretia gigantea \\
\hline & $\begin{array}{l}\text { Puya spathacea } \\
\text { (Griseb.) Mez }\end{array}$ & Adelaide, 1959 & 1991 & no & 2 synonyms \\
\hline & $\begin{array}{l}\text { Vriesea imperialis } \\
\text { Carrière }\end{array}$ & Bruxelles, 1965 & 1969 (1975) & no & 3 synonyms \\
\hline & $\begin{array}{l}\text { Vriesea saundersii } \\
\text { (Carrière) E. Morren } \\
\text { ex Mez }\end{array}$ & Bruxelles, 1966 & $\begin{array}{l}\text { no further } \\
\text { record }\end{array}$ & no & 3 synonyms \\
\hline & $\begin{array}{l}\text { Werauhia gigantea } \\
\text { (Mart. ex Schult. f.) } \\
\text { J.R. Grant }\end{array}$ & nn, 2004 & 2005 & no & $\begin{array}{l}\text { purchased; accepted name } \\
\text { is also Vriesea amazonica; } \\
\text { several synonyms }\end{array}$ \\
\hline Commelinaceae & $\begin{array}{l}\text { Aneilema acuminatum } \\
\text { R. Br. (?) }\end{array}$ & Nancy, 1981 & $2010(2013)$ & no & $\begin{array}{l}\text { arrived as "Gibasis } \\
\text { papuana", possibly } \\
\text { Aneilema papuanum, acc. to } \\
\text { Plant list, a synonym of } A \text {. } \\
\text { acuminatum }\end{array}$ \\
\hline
\end{tabular}




\begin{tabular}{|c|c|c|c|c|c|}
\hline & $\begin{array}{l}\text { Aneilema beniniense } \\
\text { (P. Beauv.) Kunth }\end{array}$ & Antwerp, 1965 & 1983 & no & \\
\hline & $\begin{array}{l}\text { Callisia elegans } \\
\text { Alexander ex H.E. } \\
\text { Moore }\end{array}$ & $\begin{array}{l}\text { Rotterdam, } 1959 \\
\& 2001\end{array}$ & $1972 \& 2008$ & no & $\begin{array}{l}\text { accepted name is also } C . \\
\text { gentlei var. elegans }\end{array}$ \\
\hline & & Strasbourg, 1972 & 1978 & no & $\begin{array}{l}\text { arrived as Setcreasea striata, } \\
\text { older synonym }\end{array}$ \\
\hline & $\begin{array}{l}\text { Callisia fragrans } \\
\text { (Lindl.) Woodson }\end{array}$ & Antwerp, 1966 & $\begin{array}{l}\text { no further } \\
\text { record }\end{array}$ & no & 2 synonyms \\
\hline & & Vacratot, 1963 & 1971 & no & \\
\hline & & Bologna, 1968 & 1969 & no & \\
\hline & $\begin{array}{l}\text { Callisia repens (Jacq.) } \\
\text { L. }\end{array}$ & nn, 1950 & 1961 & no & 3 synonyms \\
\hline & & Vacratot, 1963 & 1966 & no & \\
\hline & & Strasbourg, 1973 & 1978 & no & \\
\hline & $\begin{array}{l}\text { Coleotrype natalensis } \\
\text { C.B. Clarke }\end{array}$ & Besancon, 1965 & 1966 & no & \\
\hline & & Kaunas, 1967 & 1975 & no & \\
\hline & $\begin{array}{l}\text { Commelina } \\
\text { benghalensis L. }\end{array}$ & Tsukuba 08 & 2013 & yes & 8 synonyms; relocated \\
\hline & Commelina tuberosa L. & Graz, 1973 & 1976 & no & 8 synonyms \\
\hline & & Glasgow, 1987 & 1989 & no & \\
\hline & $\begin{array}{l}\text { Cyanotis kewensis C.B. } \\
\text { Clarke }\end{array}$ & nn, 1951 & 1961 & no & \\
\hline & & $\begin{array}{l}\text { Cluj-Napoca, } \\
1970\end{array}$ & $1970(1975)$ & no & \\
\hline & $\begin{array}{l}\text { Cyanotis nodiflora } \\
\text { (Lam.) Kunth }\end{array}$ & Kiel, 1966 & 1967 & no & $\begin{array}{l}\text { accepted name is also } \\
\text { Cyanotis speciosa }\end{array}$ \\
\hline & & Strasbourg, 1969 & 1978 & no & $\begin{array}{l}\text { arrived as Tradescantia } \\
\text { nodiflora, invalid synonym }\end{array}$ \\
\hline & $\begin{array}{l}\text { Cyanotis somaliensis } \\
\text { C.B. Clarke }\end{array}$ & nn, $1961 \& 1967$ & 1968 & no & \\
\hline & & Brno, 1963 & 1980 & no & \\
\hline & $\begin{array}{l}\text { Floscopa scandens } \\
\text { Lour. }\end{array}$ & Marburg, 1985 & 1986 & no & \\
\hline & Palisota barteri Hook. & Nancy, 1953 & 1961 & no & \\
\hline & & Vienna, 1959 & $\begin{array}{l}\text { no further } \\
\text { record }\end{array}$ & no & \\
\hline & & Erlangen, 1965 & 1975 & no & \\
\hline & & Gent, 1965 & 1973 & no & \\
\hline & & Hamburg, 1965 & 1973 & no & \\
\hline & $\begin{array}{l}\text { Palisota bracteosa C.B. } \\
\text { Clarke }\end{array}$ & Stockholm, 1958 & 1988 & no & \\
\hline & & Gent, 1965 & 1973 & no & \\
\hline & & Marburg, 1965 & 1972 & no & \\
\hline & & Nancy, 1981 & 1984 & no & \\
\hline & & $\begin{array}{l}\text { nn, in collection } \\
\text { in } 2000\end{array}$ & 2003 & no & \\
\hline & $\begin{array}{l}\text { Palisota schweinfurthii } \\
\text { C.B. Clarke }\end{array}$ & \begin{tabular}{|l|} 
Leningrad (St. \\
Petersburg), 1958
\end{tabular} & 1963 & no & $\begin{array}{l}\text { arrived as Palisota mannii, a } \\
\text { single valid synonym }\end{array}$ \\
\hline
\end{tabular}




\begin{tabular}{|c|c|c|c|c|c|}
\hline & & Nancy, 1981 & 1983 & no & \\
\hline & $\begin{array}{l}\text { Polyspatha paniculata } \\
\text { Benth. }\end{array}$ & Strasbourg, 1969 & 1975 & no & 2 synonyms \\
\hline & $\begin{array}{l}\text { Tinantia erecta (Jacq.) } \\
\text { Schltdl. }\end{array}$ & Frankfurt, 1985 & 1990 & no & 8 synonyms \\
\hline & $\begin{array}{l}\text { Tradescantia } \\
\text { amplexicaulis } \\
\text { Klotzsch ex C.B. } \\
\text { Clarke }\end{array}$ & Antwerp, 1961 & 1966 & no & $\begin{array}{l}\text { accepted name is also } \\
\text { Tripogandra amplexicaulis; } 4 \\
\text { synonyms }\end{array}$ \\
\hline & & $\begin{array}{l}\text { Cluj-Napoca, } \\
1961\end{array}$ & 1963 & no & \\
\hline & $\begin{array}{l}\text { Tradescantia pulchella } \\
\text { Kunth }\end{array}$ & $\begin{array}{l}\text { Ciudad de } \\
\text { Mexico, } 1969\end{array}$ & 1977 & no & $\begin{array}{l}\text { accepted name is also } \\
\text { Gibasis pulchella; } 2 \\
\text { synonyms }\end{array}$ \\
\hline & $\begin{array}{l}\text { Tradescantia spathacea } \\
\text { Sw. 'Vittata' }\end{array}$ & Gent, 1967 & $1972(1975)$ & no & $\begin{array}{l}\text { accepted species-names are } \\
\text { also Rhoeo spathacea and } \\
\text { T.discolor; } 4 \text { synonyms }\end{array}$ \\
\hline & $\begin{array}{l}\text { Tradescantia } \\
\text { venezuelensis } \\
\text { Steyerm. }\end{array}$ & Vacratot, 1963 & 1963 & no & $\begin{array}{l}\text { accepted name is also } \\
\text { Tradescantia gracillima; } 3 \\
\text { synonyms }\end{array}$ \\
\hline & & Atwerpen, 1967 & 1972 & no & \\
\hline & & $\begin{array}{l}\text { Besancon, } 1968 \\
\& 1977\end{array}$ & $1968 \& 1978$ & no & \\
\hline & $\begin{array}{l}\text { Tradescantia zebrina } \\
\text { Heynh. ex Bosse } \\
\text { 'Purpusii' }\end{array}$ & Nantes, 1970 & 1972 & no & $\begin{array}{l}\text { arrived as T. purpusii; } \\
\text { accepted species-names are } \\
\text { also T. pendula and Zebrina } \\
\text { pendula; } 3 \text { synonyms }\end{array}$ \\
\hline & $\begin{array}{l}\text { Tripogandra pflanzii } \\
\text { (G. Brueckn.) Rohwe- } \\
\text { der }\end{array}$ & Muenchen, 1968 & 1968 & no & $\begin{array}{l}\text { accepted names are also } T \text {. } \\
\text { glandulosa and T.radiata; } \\
\text { arrived with an invalid } \\
\text { synonym "Tradescantia } \\
\text { lanceolata" }\end{array}$ \\
\hline & $\begin{array}{l}\text { Weldenia candida } \\
\text { Schult. f. }\end{array}$ & Marburg, 1965 & $\begin{array}{l}\text { no further } \\
\text { record }\end{array}$ & no & 2 synonyms; monotypic \\
\hline \multirow[t]{2}{*}{ Costaceae } & $\begin{array}{l}\text { Costus scaber Ruiz \& } \\
\text { Pav. }\end{array}$ & Chemnitz, 2005 & 2013 & no & $\begin{array}{l}10+\text { synonyms and } \\
\text { accepted names }\end{array}$ \\
\hline & $\begin{array}{l}\text { Tapeinochilos } \\
\text { spectabilis K. Schum. }\end{array}$ & $\begin{array}{l}\text { Puerto de la } \\
\text { Cruz, } 2004\end{array}$ & 2010 & no & \\
\hline Dioscoreaceae & $\begin{array}{l}\text { Dioscorea batatas } \\
\text { Decne. }\end{array}$ & nn, 2005 & 2007 & yes & $\begin{array}{l}\text { accepted name is also } D \text {. } \\
\text { polystachya; } 5 \text { synonyms }\end{array}$ \\
\hline Doryanthaceae & $\begin{array}{l}\text { Doryanthes excelsa } \\
\text { Corrêa }\end{array}$ & Antibes, 1955 & 2010 & no & arrived as fam. Liliaceae \\
\hline \multirow[t]{7}{*}{ Iridaceae } & $\begin{array}{l}\text { Babiana ambigua G.J. } \\
\text { Lewis }\end{array}$ & $\begin{array}{l}\text { Kirstenbosch, } \\
1979\end{array}$ & $\begin{array}{l}\text { no further } \\
\text { record }\end{array}$ & no & \\
\hline & Babiana ecklonii Klatt & nn, 2004 & 2005 & no & \\
\hline & $\begin{array}{l}\text { Babiana macrantha } \\
\text { MacOwan }\end{array}$ & $\begin{array}{l}\text { Kirstenbosch, } \\
1954\end{array}$ & $\begin{array}{l}\text { no further } \\
\text { record }\end{array}$ & no & \\
\hline & $\begin{array}{l}\text { Chasmanthe bicolor } \\
\text { (Gasp. ex Ten.) N.E. } \\
\text { Br. }\end{array}$ & Blanes 2004 & 2005 & no & syn. Petamenes bicolor \\
\hline & $\begin{array}{l}\text { Herbertia pulchella } \\
\text { Sweet }\end{array}$ & $\begin{array}{l}n n \text {, in collection } \\
\text { in } 2000\end{array}$ & 2013 & no & purchased, 3 synonyms \\
\hline & & Kyoto, 2002 & 2005 & no & 4 synonyms \\
\hline & $\begin{array}{l}\text { Trimezia martinicensis } \\
\text { (Jacq.) Herb. }\end{array}$ & Bonn, 1981 & 1983 & no & 3 synonyms \\
\hline
\end{tabular}




\begin{tabular}{|c|c|c|c|c|c|}
\hline \multirow[t]{3}{*}{ Liliaceae } & $\begin{array}{l}\text { Semele androgyna (L.) } \\
\text { Kunth }\end{array}$ & Chelsea, 1953 & 1961 (1989) & no & \\
\hline & \begin{tabular}{|l|} 
Streptopus \\
amplexifolius (L.) DC.
\end{tabular} & Moscow, 1975 & 1980 & no & 8 synonyms \\
\hline & & $\begin{array}{l}\text { Meyrin, } 1973 \& \& \\
1977\end{array}$ & $\begin{array}{l}1974(1975) \& \\
1979\end{array}$ & no & \\
\hline \multirow[t]{3}{*}{ Marantaceae } & $\begin{array}{l}\text { Calathea makoyana } \mathrm{E} . \\
\text { Morren }\end{array}$ & nn, 2003 & $2003(2004)$ & no & $\begin{array}{l}\text { accepted name is also Goep } \\
\text { pertia makoyana }\end{array}$ \\
\hline & $\begin{array}{l}\text { Maranta leuconeura E. } \\
\text { Morren }\end{array}$ & nn, 1984 & 1985 (1989) & no & syn. Calathea leuconura \\
\hline & $\begin{array}{l}\text { Marantochloa flexuosa } \\
\text { (Benth.) Hutch. }\end{array}$ & Aachen, 1996 & 2003 (2005) & no & syn. Phyllodes flexuosa \\
\hline Orchidaceae & $\begin{array}{l}\text { Pleione formosana } \\
\text { Hayata 'Oriental } \\
\text { Grace' }\end{array}$ & Bern, 2002 & 2004 (2005) & no & $\begin{array}{l}\text { species is listed at CITES } \\
\text { App. II; } 7 \text { synonyms }\end{array}$ \\
\hline \multirow[t]{6}{*}{ Pandanaceae } & $\begin{array}{l}\text { Pandanus houlletii } \\
\text { Carrière }\end{array}$ & Montreal, 1956 & 1979 & no & \\
\hline & & Brisbane, 1956 & 1979 & no & \\
\hline & Pandanus lais Kurz & nn, 1968 & $\begin{array}{l}\text { no further } \\
\text { record }\end{array}$ & no & \\
\hline & $\begin{array}{l}\text { Pandanus } \\
\text { pedunculatus R. Br. }\end{array}$ & Naples, 1958 & 1961 & no & \\
\hline & & nn, 1968 & 1978 & no & \\
\hline & Pandanus sp. & Brisbane, 1956 & 1975 & no & never determined \\
\hline \multirow[t]{4}{*}{ Strelitziaceae } & $\begin{array}{l}\text { Strelitzia nicolai Regel } \\
\text { \& Koern. }\end{array}$ & Sydney, 1962 & 1963 & yes & syn. S. quensoni \\
\hline & \begin{tabular}{|l|} 
Strelitzia parvifolia \\
W.T. Aiton
\end{tabular} & Sydney, 1963 & 1972 & no & \\
\hline & & Adelaide, 1967 & 1975 & no & \\
\hline & $\begin{array}{l}\text { Strelitzia reginae } \\
\text { Aiton }\end{array}$ & nn, 1961 & $\begin{array}{l}\text { no further } \\
\text { record }\end{array}$ & yes & \\
\hline \multirow[t]{2}{*}{ Xanthorrhoeaceae } & $\begin{array}{l}\text { Geitonoplesium } \\
\text { cymosum (R. Br.) A. } \\
\text { Cunn. ex Hook. }\end{array}$ & Palermo, 1964 & 1991 & no & \\
\hline & & Genova, 1983 & 1991 & no & \\
\hline \multirow[t]{9}{*}{ Zingiberaceae } & $\begin{array}{l}\text { Alpinia arundelliana } \\
\text { (F.M. Bailey) K. } \\
\text { Schum. }\end{array}$ & $\begin{array}{l}\text { Orotava- } \\
\text { Tenerife, } 2004\end{array}$ & 2005 (2006) & no & \\
\hline & $\begin{array}{l}\text { Alpinia calcarata } \\
\text { Roscoe }\end{array}$ & nn, $1961 \& 1962$ & $\begin{array}{l}\text { no further } \\
\text { record \& } 1984 \\
\text { ("incorrect!") }\end{array}$ & no & syn. Languas calcarata \\
\hline & & Rouen, 1966 & 1982 & no & \\
\hline & & Basel, 1982 & $\begin{array}{l}\text { no further } \\
\text { record }\end{array}$ & no & \\
\hline & $\begin{array}{l}\text { Alpinia formosana } \mathrm{K} . \\
\text { Schum. }\end{array}$ & Hengchun, 2013 & 2014 & no & 4 synonyms \\
\hline & Curcuma hort. & $\begin{array}{l}\text { nn, in collection } \\
\text { in } 2000\end{array}$ & 2000 & yes & \\
\hline & Curcuma longa L. & Uppsala, 1966 & 1972 & no & $\begin{array}{l}\text { arrived as } C \text {. domestica, one } \\
\text { of } 3 \text { synonyms }\end{array}$ \\
\hline & Curcuma longa L. & Aachen, 1966 & 2010 & yes & \\
\hline & $\begin{array}{l}\text { Hedychium coccineum } \\
\text { Buch.-Ham. }\end{array}$ & Rouen 88 & 2015 & & \\
\hline
\end{tabular}


S38 Kovačić, S.: Plethora of plants - collections of the Botanical Garden, Faculty of Science, University of Zagreb (1)

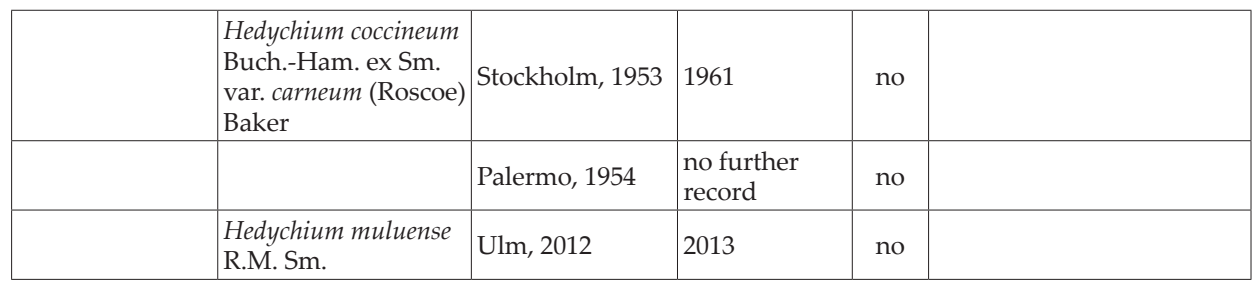


Table 3. Collection of the temperate (warm) glasshouse: recently existing plants (May, 2015)

\begin{tabular}{|c|c|c|c|}
\hline Family & $\begin{array}{l}\text { Scientific name (acc. to } \\
\text { Tropicos database) }\end{array}$ & $\begin{array}{l}\text { City of plant } \\
\text { material origin } \\
\text { (seed, cutting, bulb, } \\
\text { planta viva), year of } \\
\text { ordering/ } \\
\text { purchasing }\end{array}$ & Notes and remarks \\
\hline & SPIKEMOSSES & & \\
\hline \multirow{13}{*}{ Selaginellaceae } & Selaginella inaequalifolia Spring & Cork, 1963 & valid \\
\hline & $\begin{array}{l}\text { Selaginella involvens (Sw.) } \\
\text { Spring }\end{array}$ & \multirow{2}{*}{ Cluj-Napoca, 2015} & 3 accepted names, $20+$ synonyms \\
\hline & $\begin{array}{l}\text { Selaginella krausiana (Kunze) } \\
\text { A. Braun }\end{array}$ & & $\begin{array}{l}\text { synonyms are also S.cansecens and } \\
\text { S.denticulata }\end{array}$ \\
\hline & \multirow{2}{*}{ Selaginella martensii Spring } & Oslo, 1968 & \multirow{2}{*}{2 synonyms } \\
\hline & & Cluj-Napoca, 2015 & \\
\hline & \multirow{2}{*}{$\begin{array}{l}\text { Selaginella pallescens (C. Presl) } \\
\text { Spring }\end{array}$} & Lyon, 1966 & $\begin{array}{l}\text { arrived as S.cuspidata, one of the } \\
\text { recognized synonyms }\end{array}$ \\
\hline & & Cluj-Napoca, 2015 & $\begin{array}{l}8 \text { synonyms, among which } S \text {. } \\
\text { cuspidata and S. emmeliana }\end{array}$ \\
\hline & Selaginella pulcherrima Liebm. & Cork, 1963 & 3 synonyms \\
\hline & \multirow{3}{*}{ Selaginella tenuissima Fee } & Cluj-Napoca, 2015 & \multirow{3}{*}{$\begin{array}{l}\text { arrived as S.serpens, a valid } \\
\text { synonym }\end{array}$} \\
\hline & & Lyon, 1951 & \\
\hline & & Berlin-Dahlem, 2013 & \\
\hline & $\begin{array}{l}\text { Selaginella umbrosa Lem. ex } \\
\text { Hieron. }\end{array}$ & Cluj-Napoca, 2015 & $\begin{array}{l}\text { arrived as S. erythropus var. umbrosa; } \\
\text { syn. Is also S. e. var. major }\end{array}$ \\
\hline & Selaginella vogelii Mett. & Lyon, 1966 & $\begin{array}{l}\text { accepted name is also S.braunii; } 3 \\
\text { synonyms }\end{array}$ \\
\hline & FERNS & & \\
\hline \multirow{4}{*}{ Aspleniaceae } & Asplenium dimorphum Kunze & Berlin-Dahlem, 2003 & $\begin{array}{l}\text { unresolved name (not in Tropicos; } \\
\text { acc. to IPNI \& Plant List) }\end{array}$ \\
\hline & Asplenium nidus L. & nn, 2011 & purchased; 12 synonyms \\
\hline & $\begin{array}{l}\text { Asplenium scolopendrium L. } \\
\text { Crispum Group 'Cristatum' }\end{array}$ & nn, 2005 & $\begin{array}{l}\text { purchased as Phyllitis cristata; } \\
\text { several synonyms \& accepted } \\
\text { names of species }\end{array}$ \\
\hline & $\begin{array}{l}\text { Asplenium viviparum (L. f.) C. } \\
\text { Presl }\end{array}$ & Nancy, 1963 & $\begin{array}{l}\text { arrived as } A \text {. daucifolium var. } \\
\text { viviparum, one of } 10+\text { synonyms }\end{array}$ \\
\hline Blechnaceae & Blechnum 'Silver Lady' & nn, 2012 & $\begin{array}{l}\text { purchased; full name should be } \\
\text { "B.gibbum", but the author is } \\
\text { missing }\end{array}$ \\
\hline \multirow{5}{*}{ Davalliaceae } & $\begin{array}{l}\text { Davallia solida (G. Forst.) Sw. } \\
\text { 'Superba' }\end{array}$ & Antwerp, 1972 & $\begin{array}{l}\text { 5+ species-synonyms; accepted } \\
\text { species-name is also } D \text {. sinensis }\end{array}$ \\
\hline & Nephrolepis exaltata (L.) Schott & nn, 1954 & $\begin{array}{l}4 \text { more accepted species-names; } 20+ \\
\text { synonyms }\end{array}$ \\
\hline & $\begin{array}{l}\text { Nephrolepis exaltata (L.) Schott } \\
\text { 'Piersonii' }\end{array}$ & nn, 1961 & \\
\hline & $\begin{array}{l}\text { Nephrolepis exaltata (L.) Schott } \\
\text { 'Selecta' }\end{array}$ & Opeka, 1963 & \\
\hline & Nephrolepis 'Pearls of Living' & nn, 2012 & purchased - unknown cultivar \\
\hline
\end{tabular}




\begin{tabular}{|c|c|c|c|}
\hline & $\begin{array}{l}\text { Niphidium crassifolium (L.) } \\
\text { Lellinger }\end{array}$ & nn, 1961 & $\begin{array}{l}\text { accepted name is also Polypodium c.; } \\
10+\text { synonyms }\end{array}$ \\
\hline \multirow{3}{*}{ Dryopteridaceae } & $\begin{array}{l}\text { Bolbitis heteroclita (C. Presl) } \\
\text { Ching }\end{array}$ & Haaren, 1974 & 4 synonyms \\
\hline & $\begin{array}{l}\text { Cyrtomium falcatum (L. f.) C. } \\
\text { Presl }\end{array}$ & Stockholm, 1958 & $\begin{array}{l}\text { arrived as Polypodium } f \text {., one of } 7 \\
\text { synonyms }\end{array}$ \\
\hline & $\begin{array}{l}\text { Dryopteris pseudocaenopteris } \\
\text { (Kunze) L.B. Zhang }\end{array}$ & nn, 2009 & $\begin{array}{l}\text { purchased as Cyathea aspidioides, one } \\
\text { of } 2 \text { accepted names; } 5+\text { synonyms }\end{array}$ \\
\hline \multirow{3}{*}{ Polypodiaceae } & $\begin{array}{l}\text { Microsorum punctatum (L.) } \\
\text { Copel. }\end{array}$ & nn, 1961 & $\begin{array}{l}\text { accepted name is also } M . \text { polycarpon; } \\
60+\text { synonyms; colonizes the walls } \\
\text { inside the greenhouse }\end{array}$ \\
\hline & Polypodium aureum $\mathrm{L}$. & nn, 1963 & $\begin{array}{l}\text { accepted species-name is also } \\
\text { Phlebodium a.; } 2 \text { species-synonyms }\end{array}$ \\
\hline & $\begin{array}{l}\text { Polypodium aureum } \mathrm{L} . \\
\text { 'Umbellatum' }\end{array}$ & Essen, 1982 & \\
\hline \multirow{7}{*}{ Pteridaceae } & Adiantum capillus-veneris L. & Szeged, 1970 & $\begin{array}{l}\text { 20+ synonyms; colonizes the walls } \\
\text { inside the greenhouses }\end{array}$ \\
\hline & Adiantum raddianum $\mathrm{C}$. Presl & nn, 2012 & purchased; 15 synonyms \\
\hline & Pteris biaurita $\mathrm{L}$ & Jibou, 1984 & 7 synonyms \\
\hline & Pteris cretica L. 'Cristata' & nn, 1961 & \multirow{3}{*}{$\begin{array}{l}\text { accepted species-name is also } P \text {. } \\
\text { stenophylla; } 5 \text { synonyms }\end{array}$} \\
\hline & $\begin{array}{l}\text { Pteris cretica L. var. albolineata } \\
\text { Hook. }\end{array}$ & nn, 1961 & \\
\hline & Pteris cretica L. 'Wimsettii' & Opeka, 1963 & \\
\hline & Pteris multifida Poir. & Szeged, 1954 & 3 synonyms \\
\hline \multirow[t]{2}{*}{ Tectariaceae } & Tectaria cicutaria (L.) Copel. & $\begin{array}{l}\text { nn; plant found in } \\
\text { collection in } 2000\end{array}$ & several synonyms \\
\hline & $\begin{array}{l}\text { DICOTYLEDONS } \\
\text { (Magnoliopsida) }\end{array}$ & & \\
\hline \multirow{11}{*}{ Acanthaceae } & Beloperone guttata Brandegee & nn, 1960 & $\begin{array}{l}\text { accepted name is also Justicia } \\
\text { brandegeana; several others are } \\
\text { accepted, many synonyms }\end{array}$ \\
\hline & Crossandra nilotica Oliv. & Bonn, 1970 & \\
\hline & Crossandra pungens Lindau & Duesseldorf, 2002 & \\
\hline & Dicliptera squarrosa Nees & Antibes, $1960 \& 1973$ & $\begin{array}{l}\text { arrived as Jacobinia suberecta, one of } \\
15 \text { synonyms }\end{array}$ \\
\hline & $\begin{array}{l}\text { Elytraria caroliniensis (Walter } \\
\text { ex. F. Gmel.) Pers. }\end{array}$ & Goettingen, 2001 & syn. Tubiflora c. \\
\hline & $\begin{array}{l}\text { Elytraria caroliniensis (Walter } \\
\text { ex. F. Gmel.) Pers. var. } \\
\text { angustifolia (Fernald) S.F. } \\
\text { Blake }\end{array}$ & Havana, 1990 & several combinations of this name \\
\hline & $\begin{array}{l}\text { Fittonia albivenis (Lindl. ex } \\
\text { Veitch) Brummitt } \\
\end{array}$ & $\begin{array}{l}\text { nn; plant found in } \\
\text { collection in } 2000\end{array}$ & $\begin{array}{l}\text { several combinations of this name; } \\
\text { incl. F. verschaffeltii }\end{array}$ \\
\hline & Fittonia cult. div. & $\mathrm{nn}, 2008$ & purchased \\
\hline & $\begin{array}{l}\text { Gymnostachyum ceylanicum } \\
\text { Arn. \& Nees }\end{array}$ & Brno, 1963 & \\
\hline & $\begin{array}{l}\text { Hemigraphis alternata (Burm.f.) } \\
\text { T. Anderson cult. }\end{array}$ & Duisburg, 1989 & $\begin{array}{l}5 \text { synonyms, inc. H. colorata and } \\
\text { Ruellia coloratalalternata }\end{array}$ \\
\hline & $\begin{array}{l}\text { Hemigraphis repanda }(\mathrm{L} .) \\
\text { Hallier } \mathrm{f} .\end{array}$ & Caen, 1988 & $\begin{array}{l}\text { not in Tropicos; acc. to Plant List } \\
\text { accepted, syn of Ruellia } r \text {. }\end{array}$ \\
\hline
\end{tabular}




\begin{tabular}{|c|c|c|c|}
\hline & $\begin{array}{l}\text { Hypoestes phyllostachya Baker } \\
\text { cult. }\end{array}$ & $\begin{array}{l}\text { nn; plant found in } \\
\text { collection in } 2000\end{array}$ & \\
\hline & Justicia carnea Lindl. & Prag, 1964 & $\begin{array}{l}\text { accepted name is also Jacobinia c. } 6 \\
\text { Justicia magnifica, } 10+\text { synonyms; } \\
\text { plant was in the collection before } \\
\text { WWII }\end{array}$ \\
\hline & $\begin{array}{l}\text { Peristrophe hyssopifolia } \\
\text { (Burm.f.) Bremek. }\end{array}$ & Besancon, 1968 & $\begin{array}{l}\text { unresolved name acc. to Tropicos } \\
\text { and Plant List }\end{array}$ \\
\hline & $\begin{array}{l}\text { Phaulopsis imbricata (Forssk.) } \\
\text { Sweet }\end{array}$ & Utrecht, 1971 & 3 synonyms \\
\hline & $\begin{array}{l}\text { Pseuderanthemum alatum } \\
\text { (Nees) Radlk. }\end{array}$ & Dresden, 2002 & 2 synonyms \\
\hline & Ruellia makoyana Closon & nn, 2003 & purchased \\
\hline & Ruellia rosea (Nees) Hemsl. & Wroclaw, 1988 & 3 synonyms \\
\hline & Sanchezia nobilis Hook.f. & $\begin{array}{l}\text { nn; plant found in } \\
\text { collection in } 2000\end{array}$ & $\begin{array}{l}\text { accepted name is also } \text { S. oblonga; } 6 \\
\text { synonyms }\end{array}$ \\
\hline & $\begin{array}{l}\text { Strobilanthes reptans (G. Forst.) } \\
\text { Moylan ex Y.F. Deng \& J.R.I. } \\
\text { Wood }\end{array}$ & Muenchen, 1967 & $\begin{array}{l}\text { arrived as Hemigraphis primulifolia, } \\
\text { one of } 10+\text { synonyms }\end{array}$ \\
\hline \multirow[b]{2}{*}{ Amaranthaceae } & Aerva scandens (Roxb.) Moq. & nn, 1972 & $\begin{array}{l}\text { accepted name is also } A e . \\
\text { Sanguinolenta; } 5 \text { synonyms }\end{array}$ \\
\hline & $\begin{array}{l}\text { Pleuropetalum darwinii Hook. } \\
\text { f. }\end{array}$ & Utrecht, 2004 & $\begin{array}{l}\text { EN B1ab(ii, iii, v); B2ab(ii, iii, v) - } \\
\text { Endangered - Global and VU D2 - } \\
\text { Vulnerable - Global }\end{array}$ \\
\hline Annonaceae & Annona muricata L. & Phillipines, 2009 & 5 synonyms \\
\hline \multirow{5}{*}{ Apocynaceae } & $\begin{array}{l}\text { Cataranthus roseus (L.) G. Don } \\
\text { 'Albus' }\end{array}$ & \multirow{2}{*}{ Coimbra, 1961} & $\begin{array}{l}\text { syn. C. (roseus var.) albus; 50+ } \\
\text { synonyms }\end{array}$ \\
\hline & $\begin{array}{l}\text { Cataranthus roseus (L.) G. Don } \\
\text { 'Roseus' }\end{array}$ & & $\begin{array}{l}\text { arrived as C. (roseus var.) roseus; 50+ } \\
\text { synonyms }\end{array}$ \\
\hline & $\begin{array}{l}\text { Mandevilla splendens (Hook.) } \\
\text { Woodson }\end{array}$ & nn, 2008 & purchased; 3 synonyms \\
\hline & $\begin{array}{l}\text { Ochrosia coccinea (Teijsm. \& } \\
\text { Binn.) Miq. }\end{array}$ & Bogor, 2004 & 3 synonyms \\
\hline & \begin{tabular}{|l|} 
Trachelospermum jasminoides \\
(Lindl.) Lem.
\end{tabular} & Nanjing, 1987 & 4 synonyms \\
\hline Araliaceae & $\begin{array}{l}\text { Plerandra elegantissima (Veitch } \\
\text { ex Mast.) Lowry, G.M. } \\
\text { Plunkett \& Frodin }\end{array}$ & nn, 2010 & $\begin{array}{l}\text { purchased as Aralia e.; } 7 \text { synonyms, } \\
\text { along with Schefflera e. and } \\
\text { Dyzigotheca e. }\end{array}$ \\
\hline \multirow{3}{*}{ Aristolochiaceae } & Aristolochia gigantea Mart. & Linz, 2002 & $\begin{array}{l}\text { accepted is also A. elegans; } 2 \\
\text { synonyms }\end{array}$ \\
\hline & Aristolochia littoralis D. Parodi & $\begin{array}{l}\text { nn; plant found in } \\
\text { collection in } 2000\end{array}$ & $\begin{array}{l}\text { accepted is also } A \text {. cordiflora; } 2 \\
\text { synonyms (in collection since 1949) }\end{array}$ \\
\hline & Aristolochia zollingeriana Miq. & Hengchun, 2013 & 4 synonyms \\
\hline \multirow{5}{*}{ Begoniaceae } & Begonia acutifolia Jacq. & Potsdam, 1986 & \\
\hline & $\begin{array}{l}\text { Begonia } \times \text { erythrophylla } \\
\text { Herincq }\end{array}$ & $\begin{array}{l}\text { nn; plant found in } \\
\text { collection in } 2000\end{array}$ & B. hydrocotylifolia X B. manicata \\
\hline & Begonia fuchsioides Hook. & Nantes, 1970 & $\begin{array}{l}\text { accepted names are also B. miniata \& } \\
\text { B. foliosa var. miniata }\end{array}$ \\
\hline & $\begin{array}{l}\text { Begonia nelumbiifolia Schltdl. \& } \\
\text { Cham. }\end{array}$ & $\begin{array}{l}\text { nn; plant found in } \\
\text { collection in } 2000\end{array}$ & 4 synonyms \\
\hline & \begin{tabular}{|l|} 
Begonia peltata Otto \& D. \\
Dietr.
\end{tabular} & Vienna, 2003 & $\begin{array}{l}\text { arrived as B. kellermannii; one of } \\
\text { several synonyms }\end{array}$ \\
\hline
\end{tabular}




\begin{tabular}{|c|c|c|c|}
\hline & $\begin{array}{l}\text { Begonia } \times \text { reichenheimii } \mathrm{G} \text {. } \\
\text { Bartsch }\end{array}$ & Potsdam, 1969 & \\
\hline & Begonia 'Rex Cultorum' & nn, 2010 & purchased; various cultivars \\
\hline & Begonia $\times$ ricinifolia A. Dietr. & $\begin{array}{l}\text { nn; plant found in } \\
\text { collection in } 2000\end{array}$ & B. heracleifolia X B. peponifolia \\
\hline & $\begin{array}{l}\text { Begonia subvillosa Klotzsch } \\
\text { var. leptotricha (C. DC.) L.B. } \\
\text { Sm. \& Wassh. }\end{array}$ & Vilnius, 2013 & arrived as syn. B. leptotricha \\
\hline & $\begin{array}{l}\text { Begonia venosa Skan ex } \\
\text { Hook.f. }\end{array}$ & Braunschweig, 2002 & \\
\hline \multirow{3}{*}{ Bignoniaceae } & Crescentia cujete L. & Bonn, 1980 & 8 synonyms \\
\hline & Kigelia africana (Lam.) Benth. & Bogor, 2004 & $\begin{array}{l}\text { accepted name is also K. pinnata; } 15+ \\
\text { synonyms }\end{array}$ \\
\hline & $\begin{array}{l}\text { Pandorea jasminoides (Lindl.) } \\
\text { K. Schum. }\end{array}$ & Brisbane, 2002 & several synonyms \\
\hline Boraginaceae & Cordia caffra Sond. & Jerusalem, 2010 & \\
\hline Cucurbitaceae & Momordica rostrata A. Zimm. & Wroclaw, 1987 & \\
\hline Erythroxylaceae & Erythroxylum coca Lam. & Utrecht, 1975 & 2 synonyms \\
\hline \multirow{4}{*}{ Euphorbiaceae } & $\begin{array}{l}\text { Dalechampia roezliana Muell. } \\
\text { Arg. }\end{array}$ & Amsterdam, 1973 & $\begin{array}{l}\text { accepted name is also D. spathulata; } \\
8 \text { synonyms }\end{array}$ \\
\hline & $\begin{array}{l}\text { Euphorbia pulcherrima Willd. } \\
\text { ex Klotzsch }\end{array}$ & Sttutgart, 1970 & $\begin{array}{l}\text { arrived as unknown cultivar 'Kolle'; } \\
10+\text { synonyms }\end{array}$ \\
\hline & Jatropha curcas L. & Bogor, 1978 & $15+$ synonyms \\
\hline & $\begin{array}{l}\text { Melanolepis multiglandulosa } \\
\text { (Reinw. ex Blume) Rchb. f. \& } \\
\text { Zoll. }\end{array}$ & Hengchun, 2013 & 5 synonyms \\
\hline \multirow{3}{*}{ Fabaceae } & Bauhinia japonica Maxim. & Kyoto, 1994 & 2 synonym \\
\hline & $\begin{array}{l}\text { Hardenbergia comptoniana } \\
\text { (Andrews) Benth. }\end{array}$ & Menton, 2011 & \\
\hline & Schotia latifolia Jacq. & Melbourne, 1986 & \\
\hline \multirow{10}{*}{ Gesneriaceae } & $\begin{array}{l}\text { Alsobia dianthiflora (H.E. } \\
\text { Moore \& R.G. Wilson) } \\
\text { Wiehler }\end{array}$ & Caen, 2002 & $\begin{array}{l}\text { arrived as Episcia d., one of several } \\
\text { synonyms }\end{array}$ \\
\hline & $\begin{array}{l}\text { Episcia cupreata (Hook.) } \\
\text { Hanst. 'Silver Sheen' } \\
\end{array}$ & \multirow{2}{*}{ nn, 2003} & \multirow{2}{*}{ purchased; 2 species-synonyms } \\
\hline & $\begin{array}{l}\text { Episcia cupreata (Hook.) } \\
\text { Hanst. 'Silver Skies' } \\
\end{array}$ & & \\
\hline & Gloxinia perennis (L.) Fritsch & Goeteborg, 1978 & 15 synonyms \\
\hline & $\begin{array}{l}\text { Gloxinia sylvatica (Kunth) } \\
\text { Wiehler }\end{array}$ & Aachen, 1989 & $\begin{array}{l}\text { accepted name is also Seemannia s.; } \\
20+\text { synonyms }\end{array}$ \\
\hline & $\begin{array}{l}\text { Nematanthus gregarius D.L. } \\
\text { Denham 'Gievotz' }\end{array}$ & nn, 2002 & purchased as Nematanthus 'Gietvoz' \\
\hline & $\begin{array}{l}\text { Sinningia aggregata (Ker Gawl) } \\
\text { Wiehler }\end{array}$ & Duesseldorf, 2003 & syn. Gesneria a. \\
\hline & $\begin{array}{l}\text { Sinningia cardinalis (Lehm.) } \\
\text { H.E. Moore }\end{array}$ & Muenchen, 1978 & several synonyms \\
\hline & $\begin{array}{l}\text { Sinningia cooperi (Paxton) } \\
\text { Wiehler }\end{array}$ & Duesseldorf, 2003 & 3 synonyms \\
\hline & $\begin{array}{l}\text { Sinningia eumorpha H.E. } \\
\text { Moore }\end{array}$ & Ulm, 2012 & \\
\hline
\end{tabular}




\begin{tabular}{|c|c|c|c|}
\hline & $\begin{array}{l}\text { Sinningia speciosa (Lodd.) } \\
\text { Hiern. }\end{array}$ & Amsterdam, 1984 & \\
\hline & $\begin{array}{l}\text { Streptocarpus saxorum Engl. } \\
\text { 'Blue Velvet' }\end{array}$ & Berlin-Spaeth, 2013 & \\
\hline \multirow{8}{*}{ Lamiaceae } & $\begin{array}{l}\text { Clerodendrum cyrtophyllum } \\
\text { Turcz. }\end{array}$ & Wroclaw, 1988 & arrived as fam. Verbenaceae \\
\hline & $\begin{array}{l}\text { Clerodendrum glandulosum } \\
\text { Lindl. }\end{array}$ & Kunming, 1987 & $\begin{array}{l}\text { invalid name acc. to Tropicos; } \\
\text { accepted acc. to Plant List, with } \\
\text { several synonyms; fam. Verbenaceae }\end{array}$ \\
\hline & $\begin{array}{l}\text { Clerodendrum inerme (L.) } \\
\text { Gaertn. }\end{array}$ & Tsukuba, 1988 & $\begin{array}{l}2 \text { more accepted names; } 5+ \\
\text { synonyms; fam. Verbenaceae }\end{array}$ \\
\hline & $\begin{array}{l}\text { Clerodendrum philippinum } \\
\text { Schauer }\end{array}$ & nn, 1971 & $\begin{array}{l}\text { purchased as syn. C. fragrans } \\
\text { 'Pleniflorum' - incl. to C. } \\
\text { philippinum, with several more } \\
\text { accepted names and many } \\
\text { synonyms; as fam. Verbenaceae }\end{array}$ \\
\hline & $\begin{array}{l}\text { Clerodendrum splendens } \mathrm{G} . \\
\text { Don }\end{array}$ & Porrentruy, 1990 & arrived as fam. Verbenaceae \\
\hline & Clerodendrum ugandense Prain & nn, 2001 & purchased; fam. Verbenaceae \\
\hline & Holmskioldia sanguinea Retz. & Menton, 2009 & \\
\hline & Plectranthus prostratus Guerke & Gembloux, 2089 & \\
\hline \multirow{5}{*}{ Malvaceae } & Abroma augustum (L.) L.f. & Berlin-Dahlem, 1995 & arrived as fam. Sterculiaceae \\
\hline & $\begin{array}{l}\text { Dombeya burgessiae Gerrard ex } \\
\text { Harv. \& Sond. }\end{array}$ & Barcelona, 1990 & arrived as fam. Sterculiaceae \\
\hline & Hibiscus elatus Sw. & Graz, 1995 & 2 accepted names; several synonyms \\
\hline & Hibiscus rosa-sinensis $\mathrm{L}$. & $\begin{array}{l}\text { nn; plant found in } \\
\text { collection in } 2000\end{array}$ & \\
\hline & Theobroma cacao L. & Potsdam, 2010 & $\begin{array}{l}\text { arrived as fam. Theobromaceae; } 20 \\
\text { synonyms }\end{array}$ \\
\hline \multirow{13}{*}{ Moraceae } & Dorstenia contrajerva L. & Oxford, 1992 & $10+$ synonyms \\
\hline & Ficus benjamina $\mathrm{L}$. & Antwerp, 1969 & 5 synonyms \\
\hline & $\begin{array}{l}\text { Ficus benjamina L. 'Golden } \\
\text { Monique' }\end{array}$ & nn, 2001 & purchased; 5 species-synonyms \\
\hline & Ficus benjamina L. 'Nastasja' & nn, 2001 & purchased; 5 species-synonyms \\
\hline & Ficus binnendijkii Miq. 'Alii' & nn, 2003 & purchased \\
\hline & $\begin{array}{l}\text { Ficus elastica Roxb. ex } \\
\text { Hornem. }\end{array}$ & \multirow{2}{*}{$\begin{array}{l}\text { nn; plant found in } \\
\text { collection in } 2000\end{array}$} & 3 synonyms \\
\hline & $\begin{array}{l}\text { Ficus elastica Roxb. ex } \\
\text { Hornem. 'Decora' }\end{array}$ & & 3 species-synonyms \\
\hline & $\begin{array}{l}\text { Ficus elastica Roxb. ex } \\
\text { Hornem. 'Belize' }\end{array}$ & \multirow{3}{*}{ nn, 2001} & \multirow{3}{*}{ purchased; 3 species-synonyms } \\
\hline & $\begin{array}{l}\text { Ficus elastica Roxb. ex } \\
\text { Hornem. 'Melany' }\end{array}$ & & \\
\hline & $\begin{array}{l}\text { Ficus elastica Roxb. ex } \\
\text { Hornem. 'Petite Melany' }\end{array}$ & & \\
\hline & $\begin{array}{l}\text { Ficus fistulosa Reinw. ex } \\
\text { Blume }\end{array}$ & Hengchun, 2011 & 1 syn. \\
\hline & Ficus irisana Elmer & Hengchun, 2013 & 1 syn. \\
\hline & Ficus lyrata Warb. & $\begin{array}{l}\text { nn; plant found in } \\
\text { collection in } 2000\end{array}$ & 1 syn. \\
\hline
\end{tabular}


S44 Kovačić, S.: Plethora of plants - collections of the Botanical Garden, Faculty of Science, University of Zagreb (1)

\begin{tabular}{|c|c|c|c|}
\hline & Ficus lyrata Warb. 'Bambino' & nn, 2008 & purchased; 1 species-synonym \\
\hline & Ficus microcarpa L.f. & \multirow{2}{*}{ nn, 2001} & \multirow{2}{*}{ purchased, 15 species-synonyms } \\
\hline & Ficus microcarpa L.f. 'Formosa' & & \\
\hline & Ficus triangularis Warb. & Prag, 1964 & $\begin{array}{l}\text { acc. to Plant List, accepted name is } \\
\text { F. natalensis Hochst. ssp. leprieurii } \\
\text { (Miq.) C.C. Berg }\end{array}$ \\
\hline & Ficus pumila L. & Antwerp, 1962 & 2 synonyms \\
\hline & Ficus pumila L. 'Minima' & Linz, 1994 & \multirow{2}{*}{ cutting; 2 species-synonyms } \\
\hline & Ficus pumila L. 'Variegata' & Turku, 1989 & \\
\hline & Ficus racemosa $\mathrm{L}$. & nn, 1966 & 2 synonyms \\
\hline & $\begin{array}{l}\text { Ficus rubiginosa Desf. ex Vent. } \\
\text { 'Variegata' }\end{array}$ & Brijuni Croatia, 1963 & 1 syn. \\
\hline & Ficus septica Burm. f. & Hengchun, 2011 & 4 synonyms \\
\hline & Ficus tinctoria G. Forst. & Hengchun, 2007 & \\
\hline Oleaceae & Jasminum officinale L. & $\begin{array}{l}\text { nn; plant found in } \\
\text { collection in } 2000\end{array}$ & \\
\hline \multirow{3}{*}{ Oxalidaceae } & Biophytum sensitivum (L.) DC. & Berlin-Dahlem, 2004 & $\begin{array}{l}\text { accepted name is also } B \text {. } \\
\text { umbraculum; several synonyms }\end{array}$ \\
\hline & Oxalis lasiandra Zucc. & Berlin-Spaeth, 2013 & bulbs; 5 synonyms \\
\hline & $\begin{array}{l}\text { Oxalis triangularis A. St.-Hil. } \\
\text { 'Rubra' }\end{array}$ & Berlin-Dahlem, 2003 & bulbs; $10+$ species-synonyms \\
\hline Passifloraceae & Turnera ulmifolia L. & Joensuu, 1989 & $\begin{array}{l}9 \text { synonyms; arrived as fam. } \\
\text { Turneraceae }\end{array}$ \\
\hline \multirow{3}{*}{ Phytolaccaceae } & \multirow{3}{*}{ Rivina humilis L. } & Halle, 1983 & $\begin{array}{l}\text { arrived as } R . \text { aurantiaca; one of } 35+ \\
\text { synonyms }\end{array}$ \\
\hline & & Giesen, 1990 & $\begin{array}{l}\text { arrived as R. brasiliensis; one of 35+ } \\
\text { synonyms }\end{array}$ \\
\hline & & Frankfurt, 2014 & $\begin{array}{l}\text { 35+ synonyms; arrived as fam. } \\
\text { Riviniaceae }\end{array}$ \\
\hline \multirow{12}{*}{ Piperaceae } & Peperomia alata Ruiz \& Pav. & Vienna, 1961 & 15 synonyms \\
\hline & Peperomia blanda (Jacq.) Kunth & Wroclaw, 1989 & $\begin{array}{l}\text { arrived as P. langsdorffii, one of } 40 \\
\text { synonyms }\end{array}$ \\
\hline & $\begin{array}{l}\text { Peperomia caperata Yunck. } \\
\text { 'Rubra' }\end{array}$ & Caen, 1988 & cutting \\
\hline & $\begin{array}{l}\text { Peperomia caperata Yunck. } \\
\text { 'Variegata' }\end{array}$ & nn, 2002 & purchased \\
\hline & $\begin{array}{l}\text { Peperomia clusiifolia (Jacq.) } \\
\text { Hook. }\end{array}$ & \multirow{2}{*}{ Cluj-Napoca, 1966} & $\begin{array}{l}\text { arrived as P. obtusifolia var. } \\
\text { clusiaefolia, one of } 2 \text { synonyms }\end{array}$ \\
\hline & $\begin{array}{l}\text { Peperomia clusiifolia (Jacq.) } \\
\text { Hook. 'Variegata' }\end{array}$ & & $\begin{array}{l}\text { spontaneously, in some newly } \\
\text { vegetatively reproduced plants }\end{array}$ \\
\hline & Peperomia fenzlei Regel & Stockholm, 1969 & \\
\hline & Peperomia flexicaulis Wawra & Duisburg, 1990 & \\
\hline & $\begin{array}{l}\text { Peperomia glabella (Sw.) A. } \\
\text { Dietr. }\end{array}$ & Stockholm, 1960 & 35 synonyms \\
\hline & $\begin{array}{l}\text { Peperomia incana (Haw.) A. } \\
\text { Dietr. }\end{array}$ & $\begin{array}{l}\text { nn; plant found in } \\
\text { collection in } 2000\end{array}$ & \\
\hline & Peperomia longispicata C. DC. & Rome, 1960 & \\
\hline & $\begin{array}{l}\text { Peperomia obtusifolia (L.) A. } \\
\text { Dietr. ‘Variegata' }\end{array}$ & Napoli, 1963 & \\
\hline
\end{tabular}




\begin{tabular}{|c|c|c|c|}
\hline & Peperomia orba Bunting & Dijon, 1993 & $\begin{array}{l}\text { not in Tropicos; unresolved name } \\
\text { acc. to Plant List }\end{array}$ \\
\hline & $\begin{array}{l}\text { Peperomia pseudovariegata C. } \\
\text { DC. }\end{array}$ & \multirow{2}{*}{$\begin{array}{l}\text { Vienna-Belvedere, } \\
2012\end{array}$} & 3 synonyms \\
\hline & Peperomia reticulata Balf. f. & & \\
\hline & Peperomia rubella Hook. & Iasi, 1961 & \\
\hline & $\begin{array}{l}\text { Peperomia serpens (Sw.) } \\
\text { Loudon 'Variegata' }\end{array}$ & Besancon, 1971 & $\begin{array}{l}\text { arrived as } P \text {. serpens f. variegata, one } \\
\text { of } 25+\text { synonyms }\end{array}$ \\
\hline & Peperomia trifolia (L.) A. Dietr. & Caen, 2002 & 3 synonyms \\
\hline & $\begin{array}{l}\text { Peperomia urocarpa Fisch. \& } \\
\text { C.A. Mey. }\end{array}$ & Besancon, 1971 & 15 synonyms \\
\hline & Peperomia urvilleana A. Rich. & Berlin-Dahlem, 1991 & \\
\hline & Piper apiculatum C. DC. & Antwerp, 1966 & $\begin{array}{l}\text { accepted name is also } P \text {. divaricatum; } \\
\text { one of } 30+\text { synonyms }\end{array}$ \\
\hline & Piper arboreum Aubl. & Joensuu, 1989 & 40 synonyms \\
\hline & Piper auritum Kunth & Marburg, 1966 & 13 synonyms \\
\hline & Piper cernuum Vell. & Kopenhagen, 1988 & 20 synonyms \\
\hline & Piper cubeba L.f. & Prague, 1963 & \\
\hline & Piper nigrum L. & Antwerp, 1967 & \\
\hline Plantaginaceae & $\begin{array}{l}\text { Tetranema roseum (M. Martens } \\
\text { \& Galeotti) Standl. \& Steyerm. }\end{array}$ & Haren, 1989 & arrived as fam. Scrophulariaceae \\
\hline \multirow{5}{*}{ Rubiaceae } & Coffea arabica $\mathrm{L}$ & Chemnitz, 2003 & \\
\hline & Coffea arabica L. 'Nana' & Pruhonice, 1988 & \\
\hline & Coffea mauritiana Lam. & Cluj, 2012 & 4 synonyms \\
\hline & Coffea 'Perlita' & $\begin{array}{l}\text { nn; plant found in } \\
\text { collection in } 2000\end{array}$ & unknown cultivar \\
\hline & Coffea stenophylla G. Don & Pruhonice, 1988 & \\
\hline Sapindaceae & Sapindus trifoliatus L. & Auroville, 1990 & 1 syn. \\
\hline Saxifragaceae & $\begin{array}{l}\text { Tolmiea menziesii (Pursh) Torr. } \\
\text { \& Gray }\end{array}$ & Bielefeld, 2012 & 1 syn. \\
\hline \multirow{3}{*}{ Solanaceae } & Brunfelsia americana $\mathrm{L}$. & Bogor, 1980 & \\
\hline & Brunfelsia nitida Benth. & Havana, 1990 & \\
\hline & Cestrum aurantiacum Lindl. & Innsbruck, 2011 & 8 synonyms \\
\hline \multirow{7}{*}{ Urticaceae } & Pellionia repens (Lour.) Merr. & Cluj-Napoca, 2012 & 12 synonyms \\
\hline & $\begin{array}{l}\text { Pilea cadierei Gagnep. \& } \\
\text { Guillaumin }\end{array}$ & nn, 2010 & purchased \\
\hline & Pilea depressa (Sw.) Blume & Caen, 1995 & \\
\hline & $\begin{array}{l}\text { Pilea spruceana Wedd. } \\
\text { 'Norfolk' }\end{array}$ & nn, 1981 & purchased as Pilea 'Norfolk' \\
\hline & Pilea libanensis Urb. & nn, 2012 & purchased \\
\hline & $\begin{array}{l}\text { Pilea nummulariifolia (Sw.) } \\
\text { Wedd. }\end{array}$ & Vacratot, 1963 & 1 syn. \\
\hline & Pilea peperomioides Diels & nn, 2002 & purchased as Peperomia ariifolia \\
\hline Verbenaceae & Duranta erecta $\mathrm{L}$. & Menton, 2012 & $\begin{array}{l}\text { accepted is also D. repens; } 35+ \\
\text { synonyms }\end{array}$ \\
\hline Vitaceae & Leea guineensis G. Don & nn, 1999 & $\begin{array}{l}\text { arrived as fam. Leeaceae; } 40 \\
\text { synonyms }\end{array}$ \\
\hline
\end{tabular}




\begin{tabular}{|c|c|c|c|}
\hline & Leea indica (Burm. f.) Merr. & Hengchun, 2013 & $\begin{array}{l}\text { arrived as fam. Leeaceae; } 35+ \\
\text { synonyms }\end{array}$ \\
\hline & $\begin{array}{l}\text { Tetrastigma voinierianum } \\
\text { (Baltet) Gagnep. }\end{array}$ & Ljubljana, 2002 & 2 synonyms \\
\hline & $\begin{array}{l}\text { MONOCOTYLEDONS } \\
\text { (Liliopsida) }\end{array}$ & & \\
\hline Acoracaceae & $\begin{array}{l}\text { Acorus gramineus Sol. ex Aiton } \\
\text { 'Aureovariegatus' }\end{array}$ & Košice, 1970 & $\begin{array}{l}\text { 13+ species-synonyms; as fam. } \\
\text { Araceae }\end{array}$ \\
\hline \multirow{5}{*}{ Amaryllidaceae } & Cyrtanthus elatus (Jacq.) Traub & Rotterdam, 1971 & 3 synonyms \\
\hline & $\begin{array}{l}\text { Habranthus tubispathus } \\
\text { (L'Her.) Traub }\end{array}$ & Caen, 2002 & $15+$ synonyms \\
\hline & Haemanthus albiflos Jacq. & $\begin{array}{l}\text { nn; plant found in } \\
\text { collection in } 2000\end{array}$ & $\begin{array}{l}\text { illegitimate name acc. to Tropicos; } \\
\text { accepted acc. to Plant List }\end{array}$ \\
\hline & $\begin{array}{l}\text { Hymenocallis speciosa (Salisb.) } \\
\text { Salisb. }\end{array}$ & Amsterdam, 1970 & \\
\hline & $\begin{array}{l}\text { Sprekelia formosissima (L.) } \\
\text { Herb. }\end{array}$ & Basel, 1958 & 7 synonyms \\
\hline \multirow{4}{*}{ Araceae } & $\begin{array}{l}\text { Anthurium cordifolium (Raf.) } \\
\text { Kunth }\end{array}$ & Bucarest, 1984 & $\begin{array}{l}\text { accepted name is also } A \text {. } \\
\text { grandiflorum; several synonyms }\end{array}$ \\
\hline & $\begin{array}{l}\text { Anthurium hybridum hort. ex } \\
\text { Engl. }\end{array}$ & nn, 2004 \& 2009 & purchased; unknown cultivars \\
\hline & Ariopsis peltata J. Graham & Kiel, 1970 & \\
\hline & Philodendron hort. & nn, 2010 & purchased; unknown cultivar \\
\hline \multirow{17}{*}{ Asparagaceae } & Asparagus stipularis Forssk. & Berlin-Dahlem, 2002 & $\begin{array}{l}\text { acc. to Plant List, several accepted } \\
\text { names and synonyms }\end{array}$ \\
\hline & Aspidistra elatior Blume & nn, 1981 & fam. Liliaceae; 2 synonyms \\
\hline & $\begin{array}{l}\text { Bowiea kilimandscharica } \\
\text { Mildbr. }\end{array}$ & Basel, 1974 & $\begin{array}{l}\text { syn. B. volubilis ssp. volubilis; fam. } \\
\text { Liliaceae }\end{array}$ \\
\hline & Bowiea volubilis Harv. & Halle, 1961 & many synonyms; fam. Liliaceae \\
\hline & Chlorophytum amaniense Engl. & Menton, 2012 & arrived as fam. Anthericaceae \\
\hline & $\begin{array}{l}\text { Chlorophytum comosum } \\
\text { (Thunb.) Jacques }\end{array}$ & $\begin{array}{l}\text { nn; plant found in } \\
\text { collection in } 2000\end{array}$ & syn. Anthericum c. \\
\hline & $\begin{array}{l}\text { Chlorophytum comosum } \\
\text { (Thunb.) Jacques 'Variegatum' }\end{array}$ & Szeged, 1963 & fam. Liliaceae \\
\hline & $\begin{array}{l}\text { Chlorophytum macrophyllum (A. } \\
\text { Rich.) Asch. }\end{array}$ & Joensuu, 1989 & fam. Liliaceae \\
\hline & $\begin{array}{l}\text { Chlorophytum orchidastrum } \\
\text { Lindl. }\end{array}$ & Innsbruck, 2004 & $\begin{array}{l}\text { acc. to Plant List, several accepted } \\
\text { names and synonyms }\end{array}$ \\
\hline & Cordyline terminalis (L.) Kunth & Adelaide, 1971 & fam. Liliaceae; 4 synonyms \\
\hline & $\begin{array}{l}\text { Dasylirion acrotrichum } \\
\text { (Schiede) Zucc. }\end{array}$ & Lyon, 1960 & 3 synonyms; fam. Liliaceae \\
\hline & \multirow{2}{*}{ Dasylirion longissiumum Lem. } & Berlin-Dahlem, 1981 & \multirow{3}{*}{ arrived as fam. Liliaceae } \\
\hline & & Barcelona, 1961 & \\
\hline & $\begin{array}{l}\text { Dracaena aletriformis (Haw.) } \\
\text { Bos }\end{array}$ & nn, 1961 & \\
\hline & Dracaena draco (L.) L. & Ariana, 1986 & several synonyms; fam. Liliaceae \\
\hline & Dracaena draco (L.) L. 'Sydney' & nn, 2010 & purchased \\
\hline & $\begin{array}{l}\text { Dracaena fragrans (L.) } \\
\text { Ker-Gawl. }\end{array}$ & nn, 1961 & $\begin{array}{l}\text { arrived as } D . \text { deremensis; } 3 \\
\text { synonyms; fam. Liliaceae }\end{array}$ \\
\hline
\end{tabular}




\begin{tabular}{|c|c|c|c|}
\hline & $\begin{array}{l}\text { Dracaena fragrans (L.) } \\
\text { Ker-Gawl. var. warneckei Engl. }\end{array}$ & $\begin{array}{l}\text { Lada Nursery } \\
\text { Slovenia, } 1964\end{array}$ & $\begin{array}{l}\text { purchased as D. warneckii } \\
\text { (unknown/illegitimate); many } \\
\text { synonyms; fam. Liliaceae }\end{array}$ \\
\hline & $\begin{array}{l}\text { Dracaena fragrans (L.) } \\
\text { Ker-Gawl. 'Yellow Coast' }\end{array}$ & \multirow[t]{2}{*}{ nn, 2010} & \multirow{5}{*}{ purchased } \\
\hline & Dracaena marginata hort. & & \\
\hline & $\begin{array}{l}\text { Dracaena reflexa Lam. 'Gold } \\
\text { Coast' }\end{array}$ & \multirow{3}{*}{ nn, 2001} & \\
\hline & $\begin{array}{l}\text { Dracaena reflexa Lam. 'Lemon } \\
\text { Surprise' }\end{array}$ & & \\
\hline & $\begin{array}{l}\text { Dracaena reflexa Lam. 'Song of } \\
\text { India' }\end{array}$ & & \\
\hline & $\begin{array}{l}\text { Drimiopsis maculata Lindl. \& } \\
\text { Paxton }\end{array}$ & Coimbra,1961 & $\begin{array}{l}\text { fam. Liliaceae; acc. to Plant List, } \\
\text { several accepted names and } \\
\text { synonyms }\end{array}$ \\
\hline & $\begin{array}{l}\text { Eucomis comosa (Houtt.) } \\
\text { Wehrh. }\end{array}$ & Sienna, 1984 & $\begin{array}{l}\text { arrived as E. punctata; many } \\
\text { synonyms; fam. Liliaceae }\end{array}$ \\
\hline & Eucomis regia (L.) Aiton & Palermo, 1961 & 3 synonyms; fam. Liliaceae \\
\hline & \multirow{3}{*}{$\begin{array}{l}\text { Nolina recurvata (Lem.) } \\
\text { Hemsl. }\end{array}$} & Palermo, 1969 & \multirow{3}{*}{$\begin{array}{l}\text { fam. Liliaceae; accepted name is also } \\
\text { Beaucarnea r.; acc.to Plant List, } \\
\text { several synonyms }\end{array}$} \\
\hline & & Montreal, 1986 & \\
\hline & & Menton, 2014 & \\
\hline & Sansevieria arborescens Gentil & Budapest, 1978 & fam. Liliaceae \\
\hline & $\begin{array}{l}\text { Sansevieria cylindrica Bojer ex } \\
\text { Hook. }\end{array}$ & Brno, 1963 & fam. Liliaceae \\
\hline & Sansevieria dooneri N.E. Br. & Goeteborg, 1978 & fam. Liliaceae \\
\hline & Sansevieria grandicuspis Haw. & Goeteborg, 1978 & fam. Liliaceae \\
\hline & Sansevieria grandis Hook.f. & $\begin{array}{l}\text { nn; plant found in } \\
\text { collection in } 2000\end{array}$ & fam. Liliaceae \\
\hline & $\begin{array}{l}\text { Sansevieria metallica Gerome \& } \\
\text { Labroy }\end{array}$ & Budapest, 1975 & $\begin{array}{l}\text { accepted name is also } S . \\
\text { hyacinthoides; } 12 \text { synonyms; fam. } \\
\text { Liliaceae }\end{array}$ \\
\hline & Sansevieria raffillii N.E. Br. & Budapest, 1977 & fam. Liliaceae \\
\hline & Sansevieria stuckyi God.-Leb. & Rome, 1960 & fam. Liliaceae \\
\hline & Sansevieria trifasciata Prayn & Szeged, 1970 & 5 synonyms; fam. Liliaceae \\
\hline & $\begin{array}{l}\text { Sansevieria trifasciata Prayn } \\
\text { 'Golden Hahnii' }\end{array}$ & Wroclaw, 1986 & fam. Liliaceae \\
\hline & $\begin{array}{l}\text { Sansevieria trifasciata Prayn } \\
\text { 'Gruene Craigii' }\end{array}$ & Wroclaw, 1986 & fam. Liliaceae \\
\hline & $\begin{array}{l}\text { Sansevieria trifasciata Prayn } \\
\text { 'Hahnii' }\end{array}$ & nn, 1961 & fam. Liliaceae \\
\hline & $\begin{array}{l}\text { Sansevieria trifasciata Prayn } \\
\text { 'Laurentii' }\end{array}$ & nn, 1963 & fam. Liliaceae \\
\hline & $\begin{array}{l}\text { Sansevieria trifasciata Prayn } \\
\text { 'Silver Hahnii' }\end{array}$ & Kiev, 1989 & fam. Liliaceae \\
\hline Bromeliaceae & $\begin{array}{l}\text { Acanthostachys strobilacea } \\
\text { (Schult. f.) Link, Klotzsch \& } \\
\text { Otto }\end{array}$ & Muenchen, 1963 & 2 synonyms \\
\hline & Aechmea ‘Blue Rain’ & nn, 2010 & purchased \\
\hline
\end{tabular}




\begin{tabular}{|c|c|c|}
\hline Aechmea bracteata (Sw.) Griseb. & Frankfurt, 1957 & $10+$ synonyms \\
\hline $\begin{array}{l}\text { Aechmea bromeliifolia (Rudge) } \\
\text { Baker }\end{array}$ & Marburg, 1965 & $25+$ synonyms \\
\hline Aechmea fasciata (Lindl.) Baker & nn, 1977 & \\
\hline $\begin{array}{l}\text { Aechmea lindenii (E. Morren) } \\
\text { Baker var. makoyana Mez }\end{array}$ & Marburg, 1965 & $\begin{array}{l}\text { accepted name is also Ae. comata; } \\
\text { several synonyms }\end{array}$ \\
\hline $\begin{array}{l}\text { Aechmea lindenii (E. Morren) } \\
\text { Baker 'Variegata' }\end{array}$ & Brisel, 1965 & $\begin{array}{l}\text { arrived as Ae. comata var. variegata; } \\
\text { accepted species-name is also Ae. } \\
\text { comata }\end{array}$ \\
\hline $\begin{array}{l}\text { Aechmea lueddemanniana (K. } \\
\text { Koch) Brongn. ex Mez }\end{array}$ & Prag, 1964 & 10 synonyms \\
\hline $\begin{array}{l}\text { Aechmea mariae-reginae } \mathrm{H} . \\
\text { Wendl. }\end{array}$ & Hamburg, 1967 & 3 synonyms \\
\hline Aechmea penduliflora Andre & Nantes, 1985 & 4 synonyms \\
\hline $\begin{array}{l}\text { Aechmea recurvata (Klotzsch) } \\
\text { L.B. Sm. }\end{array}$ & nn, 1995 & \\
\hline Aechmea sphaerocephala Baker & Berlin-Dahlem, 1965 & 3 synonyms \\
\hline Billbergia alfonsi-joannis Reitz & Cluj-Napoca, 2002 & \\
\hline $\begin{array}{l}\text { Billbergia brasiliensis (hort. ex } \\
\text { Lem.) L.B. Sm. }\end{array}$ & Cluj-Napoca, 1965 & \\
\hline $\begin{array}{l}\text { Billbergia iridifolia (Nees \& } \\
\text { Mart.) Lindl. }\end{array}$ & $\begin{array}{l}\text { nn; plant found in } \\
\text { collection in } 2000\end{array}$ & \\
\hline Billbergia kuhlmannii L.B. Sm. & Vacratot, 2009 & several synonyms \\
\hline Billbergia macrolepis L.B. Sm. & Cluj-Napoca, 2004 & $\begin{array}{l}3 \text { synonyms; VU D2 - Vulnerable - } \\
\text { National (Colombia) }\end{array}$ \\
\hline $\begin{array}{l}\text { Billbergia nutans H. Wendl ex } \\
\text { Regel }\end{array}$ & Nantes, 1959 & \\
\hline Billbergia rosea hort. ex Beer & Berlin-Dahlem, 1968 & $\begin{array}{l}\text { arrived as B. venezuelana, one of } \\
\text { several synonyms }\end{array}$ \\
\hline $\begin{array}{l}\text { Billbergia saundersii hort. ex } \\
\text { Dombrain }\end{array}$ & Prague, 1964 & $\begin{array}{l}\text { accepted name is also } B \text {. chlorosticta } \\
\text { \& Neoregelia ch.; } 10+\text { synonyms }\end{array}$ \\
\hline $\begin{array}{l}\text { Billbergia vittata Brong. ex } \\
\text { Morel }\end{array}$ & Liverpool, 1962 & 10 synoynms \\
\hline $\begin{array}{l}\text { Billbergia zebrina (Herb.) } \\
\text { Lindl. }\end{array}$ & Basel, 1965 & $\begin{array}{l}\text { arrived as (also accepted name) } B . \\
\text { porteana; } 10+\text { synonyms }\end{array}$ \\
\hline $\begin{array}{l}\text { Billbergia zebrina (Herb.) } \\
\text { Lindl. }\end{array}$ & Utrecht, 2004 & $\begin{array}{l}\text { accepted name is also } B . \text { porteana; } \\
10+\text { synonyms }\end{array}$ \\
\hline $\begin{array}{l}\text { Cryptanthus acaulis (Lindl.) } \\
\text { Beer }\end{array}$ & Berlin-Dahlem, 2003 & \\
\hline $\begin{array}{l}\text { Deuterocohnia meziana Kuntze } \\
\text { ex Mez }\end{array}$ & Dresden, 2012 & 3 synonyms \\
\hline Dyckia niederleinii $\mathrm{Mez}$ & Nantes, 2009 & 2 synonyms \\
\hline $\begin{array}{l}\text { Fosterella penduliflora (C.H. } \\
\text { Wright) L.B. Sm. }\end{array}$ & Brno, 2012 & 4 synonyms \\
\hline $\begin{array}{l}\text { Guzmania lingulata (L.) Mez } \\
\text { cult. }\end{array}$ & nn, 2010 & $\begin{array}{l}\text { purchased, several cultivars (red, } \\
\text { yellow and orange) }\end{array}$ \\
\hline $\begin{array}{l}\text { Neoregelia spectabilis (Moore) } \\
\text { L.B.Sm. }\end{array}$ & Prague, 1964 & 5 synonyms, diff. genera \\
\hline Nidularium "candidum" (?) & $\begin{array}{l}\text { nn; plant found in } \\
\text { collection in } 2000\end{array}$ & unknown taxon \\
\hline Orthophytum foliosum L.B. Sm. & Potsdam, 2009 & \\
\hline Pitcairnia grafii Rauh & Utrecht, 2004 & \\
\hline
\end{tabular}




\begin{tabular}{|c|c|c|c|}
\hline & Puya chilensis Molina & Balchik, 2008 & 10 synonyms \\
\hline & Puya mirabilis (Mez.) LB.Sm. & Nantes, 1986 & 2 synonyms \\
\hline & Puya spathacea (Grieseb.) Mez. & Adelaide, 1959 & 2 synonyms \\
\hline & $\begin{array}{l}\text { Vriesea splendens hort. ex M.B. } \\
\text { Foster }\end{array}$ & nn, 2010 & purchased \\
\hline \multirow{2}{*}{ Colchicaceae } & Gloriosa superba L. & Pretoria, 1981 & $\begin{array}{l}\text { arrived as fam. Liliaceae; } 2 \\
\text { synonyms }\end{array}$ \\
\hline & $\begin{array}{l}\text { Gloriosa superba L. } \\
\text { 'Rothschildiana' }\end{array}$ & Bergen, 1970 & arrived as fam. Liliaceae \\
\hline \multirow{14}{*}{ Commelinaceae } & $\begin{array}{l}\text { Aclisia condensata (C.B. } \\
\text { Clarke) G. Brueckn. }\end{array}$ & Nancy, 1992 & $\begin{array}{l}\text { acc. to Plant List, accepted name is } \\
\text { also Pollia } \text {. }\end{array}$ \\
\hline & Callisia insignis C.B. Clarke & Antwerp, 1978 & \\
\hline & $\begin{array}{l}\text { Callisia multiflora (M. Martens } \\
\text { \& Galeotti) Standl. }\end{array}$ & Strassbourg, 1973 & 5 synonyms \\
\hline & $\begin{array}{l}\text { Cyanotis vaga (Lour.) Schult. \& } \\
\text { Schult.f. }\end{array}$ & Bucarest, 1958 & $\begin{array}{l}\text { arrived without an author; many } \\
\text { synonyms and accepted names }\end{array}$ \\
\hline & $\begin{array}{l}\text { Murdannia nudiflora (L.) } \\
\text { Brennan }\end{array}$ & Tsukuba, 2004 & 10 synonyms \\
\hline & Setcreasea purpurea Boom & Bonn, 1964 & $\begin{array}{l}\text { accepted name is also Tradescantia } \\
\text { pallida; many synonyms }\end{array}$ \\
\hline & Tradescantia albiflora Kunth & nn, 1961 & $\begin{array}{l}\text { accepted name is also } T \text {. fluminensis; } \\
3 \text { synonyms }\end{array}$ \\
\hline & Tradescantia geniculata Jacq. & Marburg, 1965 & $\begin{array}{l}\text { accepted names are also Gibasis g. \& } \\
\text { Aneilema g. }\end{array}$ \\
\hline & $\begin{array}{l}\text { Tradescantia sillamontana } \\
\text { Matuda }\end{array}$ & Kaunas, 2012 & 1 synonyms \\
\hline & Tradescantia spathacea Sw. & Nancy, 1970 & \multirow{2}{*}{$\begin{array}{l}\text { accepted species-names are also } T \text {. } \\
\text { discolor \& Rhoeo spathacea; many } \\
\text { synonyms }\end{array}$} \\
\hline & $\begin{array}{l}\text { Tradescantia spathacea Sw. } \\
\text { 'Variegata' }\end{array}$ & $\begin{array}{l}\text { nn; plant found in } \\
\text { collection in } 2000\end{array}$ & \\
\hline & $\begin{array}{l}\text { Tradescantia zebrina Heynh. ex } \\
\text { Bosse }\end{array}$ & nn, 1961 & $\begin{array}{l}\text { also Zebrina pendula \& Z. purpusii; } \\
\text { many synonyms }\end{array}$ \\
\hline & $\begin{array}{l}\text { Tradescantia zebrina Heynh. ex } \\
\text { Bosse 'Quadricolor' }\end{array}$ & $\begin{array}{l}\text { nn; plant found in } \\
\text { collection in } 2000\end{array}$ & \\
\hline & $\begin{array}{l}\text { Tradescantia zebrina Heynh. ex } \\
\text { Bosse var. flocculosa (G. } \\
\text { Brueckn.) D.R. Hunt }\end{array}$ & Strassbourg, 1973 & \\
\hline \multirow{4}{*}{ Costaceae } & Costus afer Ker-Gawl. & Meise, 1989 & accepted name is also C. maculatus \\
\hline & Costus deistelii K. Schum. & Graz, 2013 & \\
\hline & Costus megalobractea K. Schum. & Duesseldorf, 2012 & \\
\hline & Costus spiralis (Jacq.) Roscoe & Bogor, 2004 & $\begin{array}{l}\text { accepted name is also C. woodsonii; } \\
\text { syn. Alpinia s. }\end{array}$ \\
\hline Dioscoreaceae & Tacca chantrieri Andre & Dresden, 2012 & 4 synonyms \\
\hline \multirow{5}{*}{ Iridaceae } & Aristea ecklonii Baker & Jerusalem, 2012 & \\
\hline & $\begin{array}{l}\text { Dietes iridioides (L.) Sweet \& } \\
\text { Klatt }\end{array}$ & Cluj-Napoca, 2002 & 3 synonyms \\
\hline & $\begin{array}{l}\text { Gladiolus callianthus Marais } \\
\text { ‘Murielae' }\end{array}$ & Berlin-Dahlem, 2003 & \\
\hline & $\begin{array}{l}\text { Neomarica gracilis (Herb.) } \\
\text { Sprague }\end{array}$ & Bruxelles, 1960 & 2 synonyms \\
\hline & $\begin{array}{l}\text { Orthrosanthus multiflorus } \\
\text { Sweet }\end{array}$ & Chelsea, 2012 & \\
\hline
\end{tabular}




\begin{tabular}{|c|c|c|c|}
\hline Liliaceae & Ledebouria socialis Jessop & Uppsala, 1964 & $\begin{array}{l}\text { arrived as Scilla violacea (syn. } S . \\
\text { socialis) }\end{array}$ \\
\hline Marantaceae & Maranta arundinacea $\mathrm{L}$. & Potsdam, 1986 & \\
\hline Orchidaceae & $\begin{array}{l}\text { Ludisia discolor (Ker-Gawl.) A. } \\
\text { Rich. }\end{array}$ & Berlin-Dahlem, 2003 & \\
\hline Poaceae & $\begin{array}{l}\text { Setaria palmifolia (J. Koenig) } \\
\text { Stapf }\end{array}$ & Ulm, 2012 & $40+$ synonyms \\
\hline \multirow{3}{*}{ Strelitziaceae } & Strelitzia alba Skeels & $\begin{array}{l}\text { Puerto de la Cruz, } \\
1999\end{array}$ & $\begin{array}{l}2 \text { synonyms; acc. to Plant list, the } \\
\text { author is (L.f.) Skeels }\end{array}$ \\
\hline & Strelitzia reginae Aiton & $\begin{array}{l}\text { Puerto de la Cruz, } \\
1982\end{array}$ & \multirow{2}{*}{$\begin{array}{l}\text { acc. to Plant list, the author of } \\
\text { species is Banks and there are many } \\
\text { synonyms }\end{array}$} \\
\hline & $\begin{array}{l}\text { Strelitzia reginae Aiton } \\
\text { 'Mandela's Gold' }\end{array}$ & Kirstenbosch, 1997 & \\
\hline Xanthorrhoeaceae & Dianella tasmanica Hook. f. & Chelsea, 2012 & \\
\hline \multirow{8}{*}{ Zingiberaceae } & $\begin{array}{l}\text { Alpinia speciosa (Blume) D. } \\
\text { Dietr. }\end{array}$ & Menton, 2008 & $\begin{array}{l}\text { accepted name is also Etlingera } \\
\text { elatior; } 10 \text { synonyms }\end{array}$ \\
\hline & $\begin{array}{l}\text { Alpinia zerumbet (Pers.) B.L. } \\
\text { Burtt \& R.M. Sm. 'Variegata' }\end{array}$ & nn, 2010 & purchased as A. speciosa 'Variegata' \\
\hline & $\begin{array}{l}\text { Brachichylum horsfieldii (R. Br. } \\
\text { ex Wall.) Petersen }\end{array}$ & Utrecht, 1963 & $\begin{array}{l}\text { acc. to Plant List, accepted name is } \\
\text { also Hedychium } h . \text {, and several } \\
\text { others }\end{array}$ \\
\hline & Curcuma cult. & nn, 2003 & purchased \\
\hline & $\begin{array}{l}\text { Elettaria cardamomum (L.) } \\
\text { Maton }\end{array}$ & Gent, 1988 & 2 synonyms \\
\hline & $\begin{array}{l}\text { Hedychium coccineum } \\
\text { Buch.-Ham. ex Sm. }\end{array}$ & Rouen, 1988 & \multirow{2}{*}{ acc. to Plant List, several synonyms } \\
\hline & $\begin{array}{l}\text { Hedychium gardnerianum } \\
\text { Sheppard ex Ker Gawl. }\end{array}$ & Ulm, 2012 & \\
\hline & Zingiber officinale Roscoe & Aachen, 1987 & 7 synonyms \\
\hline
\end{tabular}

